Review

\title{
From Polymer to Small Organic Molecules: A Tight Relationship between Radical Chemistry and Solid-Phase Organic Synthesis
}

\section{Danilo Mirizzi and Maurizio Pulici *}

Department of Chemical Core Technologies, Nerviano Medical Sciences S.r.l., V.le Pasteur, 10, 20014, Nerviano (MI), Italy

* Author to whom correspondence should be addressed; E-Mail: maurizio.pulici@nervianoms.com; Tel.: +39-(0)331-581216.

Received: 8 March 2011; in revised form: 28 March 2011 / Accepted: 11 April 2011/

Published: 18 April 2011

\begin{abstract}
Since Gomberg's discovery of radicals as chemical entities, the interest around them has increased through the years. Nowadays, radical chemistry is used in the synthesis of $75 \%$ of all polymers, inevitably establishing a close relationship with Solid-Phase Organic Synthesis. More recently, the interest of organic chemists has shifted towards the application of usual "in-solution" radical chemistry to the solid-phase, ranging from the use of supported reagents for radical reactions, to the development of methodologies for the synthesis of small molecules or potential libraries. The aim of this review is to put in perspective radical chemistry, moving it away from its origin as a synthetic means for solid supports, to becoming a useful tool for the synthesis of small molecules.
\end{abstract}

Keywords: radical; solid-phase; SPS; polymer-assisted synthesis; polymer-supported reagents

Abbreviations: AIBN: Azo-bis-isobutyronitrile, AMBN: azobismethylisobutyronitrile, ATRC: Transition-metal-catalyzed atom transfer radical cyclization, 9-BBN: 9-Borabicyclo[3.3.1]nonane, CAN: Ceric ammonium nitrate, DAST: Diethylaminosulfur trifluoride, DCE: 1,2-Dichloroethane, DCM: Dichloromethane, DIC: Diisopropyl carbodiimide, DIPAD: Diisopropyl azadicarboxylate, DIPEA: diisopropylethylamine, DLP: lauroylperoxide, DMA: N,N-Dimethylacetamide, DMAP: 4-dimethylaminopyridine, DMF: N,N-Dimethylaminoformamide, EPHP: N-ethylpiperidine hypophosphite, FRET: Fluorescence Resonance Energy Transfer, HASC: $\alpha$-Hetero-Atom Substituted Carbonyl, 
HBTU: 2-(1H-Benzotriazole-1-yl)-1,1,3,3-tetramethyluronium hexafluorophosphate, HMPA: Hexamethylphosphoramide, IBX: 2-Iodoxybenzoic acid, LA: Lewis acid, NMM: N-methyl morpholine, NMP: N-methyl pyrrolidine, NMQ: N-methylquinolinium, PMHS: Polymethylhydrosiloxane, RCM: Ring-closing metathesis, SET: single electron transfer, SP: Solid-phase, SPS: Solid-phase synthesis, TBGH: Tributylgermanium hydride, TBTH: Tributyltin hydride, TEMPO: Tetramethyl pentahydropyridine oxide, TFA: Trifluoroacetic acid, THF: Tetrahydrofuran, TON: Turnover number, TTF: Tetrathiofulvalene, TTMSS: Tris(trimethylsilyl)silane, Troc: 2,2,2-Trichloroethoxycarbonyl

\section{Introduction}

Since Gomberg's discovery of free radicals as chemical entities [1], the interest around this electron-deficient species has increased throughout the years. Their success arrived during WWII, when radical chemistry took a primary role in the manufacturing of synthetic substitutes of natural rubber. Nowadays, radical processes are used to prepare $75 \%$ of all polymers, including the solid supports employed in organic synthesis.

An additional and interesting application of radical chemistry involves post polymerization modifications, which transforms the surface of the polymer for several purposes [2-4]. The change of polymers' surface characteristics allows the acquisition of new properties of the solid phase (SP) and, although this represents an interesting scientific aspect of the subject, touching the field of organic synthesis as well, it is also somewhat out of the purpose of this review and, for those interested, it would be more appropriate to refer to more specific literature.

Inevitably, all of these roles of radical chemistry have allowed creating a thread with SP synthesis. SP synthesis is an important feature in modern organic chemistry, however, the role of free radicals includes more than the methodologies just used for polymerization. The real boost came after physical organic chemists begun to determine absolute rates of radical reactions [5,6], together with the advent of new spectroscopic techniques (ESR) to detect them. At that point, radical chemistry became a more and more common methodology for day-to-day organic synthesis. With a wider range of available radical reactions, several applications in the synthesis of natural compounds, drug-like molecules and functional groups interconversion strategies were identified. It would have been just a matter of time to find extensions of those methodologies to SP approaches.

This review focuses on the applications of radical chemistry in solid-phase synthesis of single or classes of compounds, which includes also resin loading and cleavage and the use of solid-supported reagents. This subject has been last reviewed a few years ago [7]. The aim of this work is not to offer a comprehensive overview of the applications of radical chemistry on SP, but rather to emphasize the methodologies and developments in a field that might be useful in daily organic chemistry.

However, since classical radical chemistry is governed by kinetic considerations, before going through the examples that the literature offers on the subject it is fundamental to understand how radical reactions behave when they take place on the surface of a solid bead. In this respect it is important to mention Curran's work [8], dealing with the measurement of the kinetics of radical 
reactions on SPs. Curran calculated the rates of common reactions involving radical precursors anchored to an Ellman THP resin by the ratio of the possible products, in relation to the same reaction run in solution.

He found that the rate constant of $H$-abstraction from $\mathrm{Bu}_{3} \mathrm{SnH}$ by an alkyl radical linked to SP is probably not so different from the solution-phase one. This means that reactions of polymer-bound radicals, at least those mediated by tin hydride, can be planned as if they were performed in solution.

On the other hand, experiments performed with the generation of aryl radicals demonstrated that Ellman's resin behaves like a solvent with relatively reactive $\mathrm{C}-\mathrm{H}$ bonds. This means that $H$-abstraction from the polymer backbone is a feasible process, implicating that, at least in the case of the studied aryl radical, it is difficult to set up reactions whose kinetics are slow. Accordingly, for resin-bound aryl radicals there is a limitation to the fastest intramolecular classes, while slower intraand bimolecular reactions will suffer from competing hydrogen transfer from the backbone and linker C-H bonds.

\section{Solid-Supported Reagents, Resins and Linkers}

Among the various relationships between radical chemistry and SP, two interesting aspects are the use of special resins or linkers cleavable under radical conditions and solid-supported reagents designed to assist radical reactions in solution. In this regard, this section will provide a review of the literature.

\subsection{Linkers and Resins}

In the quest for reliable, clean and flexible linkers for SP synthesis, an important role is assigned to those cleavable via radical mechanisms. In particular, photolabile moieties represent the great majority of the type and several resins with appropriately designed linkers are commercially available. Since this specific subject covers more than 20 years of research and has been accurately review in the past, we refer readers to more appropriate publications $[9,10]$.

Beside light-sensitive linkers, a number of studies have treated innovative linkages based on cleavage under radical conditions. Radical cleavage of linker units usually enables the introduction of either a hydrogen atom at the cleavage site (traceless linker [11]), or, which is of even more relevance in the context of diversity oriented synthesis [12], of an additional diversity element (diversity linker unit [13]). In this section of the review, the attention will be shifted among the "key" atom in the linker.

\subsubsection{Sulfur-Based Linkers}

In the need of new methods for SP synthesis with $C-H$ bond formation after cleavage (traceless linker strategy), a linking strategy consisting in the presence of a sulfur atom, in different oxidation states susceptible of radical cleavage, was developed. The work performed by Janda [14] falls under this category (Scheme 1). 
Scheme 1. Janda's sulfur-based linker.

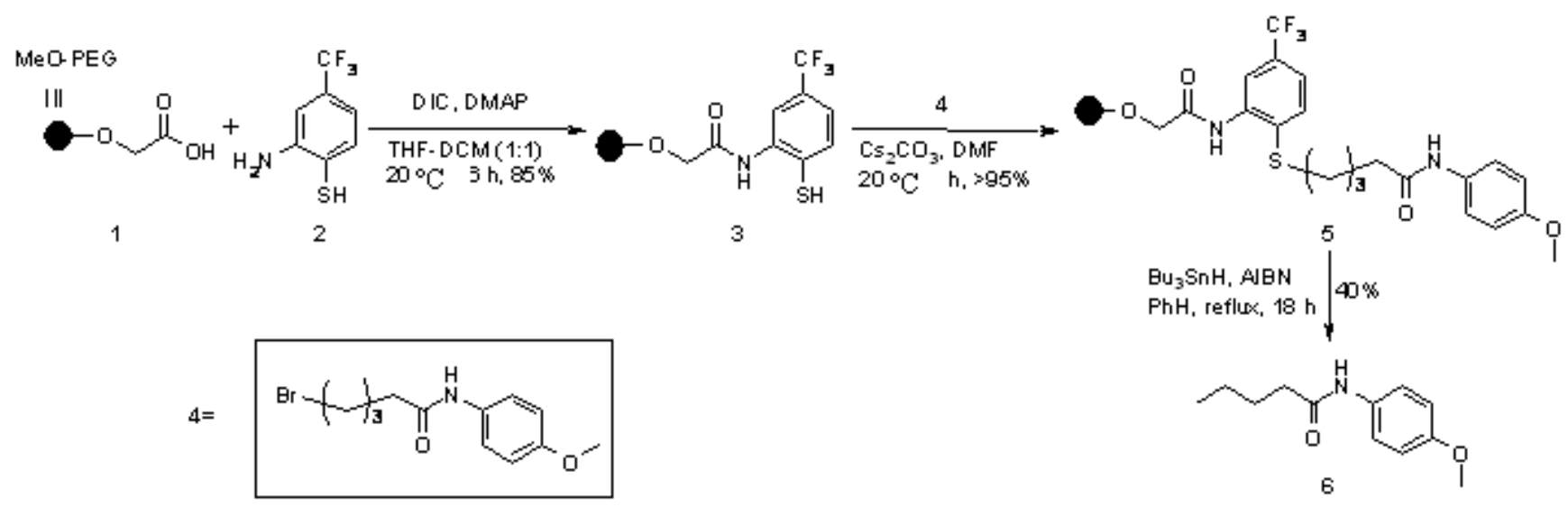

Loading of test compound $\mathbf{4}$ was performed on a soluble PEG-polymer using the pre-loaded linker 3. Reductive cleavage with $\mathrm{C}-\mathrm{H}$ bond formation was tried under classical radical conditions; however, hydrogenolysis (Ni-Raney) gave better yield (94\%) after shorter reaction time ( $3 \mathrm{~h}$ ).

In the following example, Winssinger and coworkers [15] completed the synthesis of a series of derivatives of the natural product aigialomycin $\mathrm{D}$, in order to explore the potential activities of this class of compounds (Scheme 2). Polymer-supported 8 was generated from 7 through functionalization of the benzyl carbon and subsequent RCM. Cleavage from the resin under radical conditions gave reduced products 9 .

Scheme 2. Winssinger's sulfur-based linker.

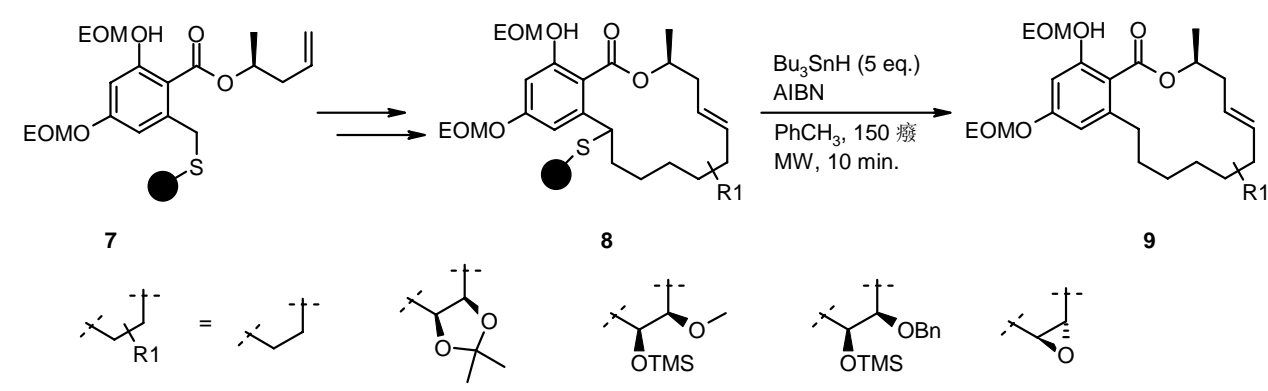

Radical desulfonation is a known reaction in solution-phase synthesis [16]. Based on this reaction, Luo and Huang [17] studied the potentiality of sulfonamide moieties as traceless linkers cleaved by radical mechanism in order to generate a library of secondary amides (Scheme 3).

Scheme 3. Huang's sulfur-based linker.

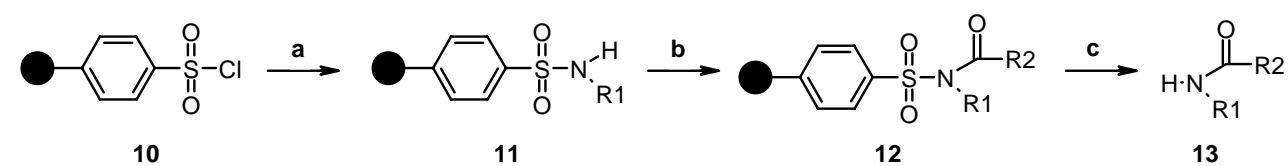

\footnotetext{
a) $\mathrm{R}^{1} \mathrm{NH}_{2}, \mathrm{Py}, 60{ }^{\circ} \mathrm{C} 2 \mathrm{~h}$; b) $\mathrm{Py}_{1} \mathrm{R}^{2} \mathrm{COCl}$, r.t., 24 h or i) $\mathrm{NaH} / \mathrm{Py}$, ii) $\left(\mathrm{R}^{2} \mathrm{CO}_{2} \mathrm{O}\right.$; c) i: TiCl/Zn, THF, refluxing, $24 \mathrm{~h}$, ii: $3 \% \mathrm{HCl}$

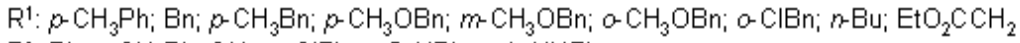

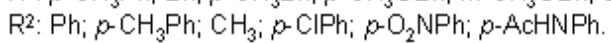


The results of the final cleavage were deeply dependent on the nature of the $\mathrm{R}^{1}$ group. Generally speaking, substituents containing electron-donating groups (such as a $m$-methoxyphenyl group) were cleaved successfully, while those containing electron-withdrawing moieties, such as a nitrophenyl group, gave no cleavage at all. In the former case, the presence of $o, p$-methoxyphenyl groups gave by-products from a competitive radical cascade, while, in the latter, reduction and acetylation of the resulting amino group were necessary to perform radical cleavage. The proposed mechanism (Scheme 4) gives an idea about how the nature of $\mathrm{R}^{1}$ can influence the radical fragmentation. In fact, if $\mathrm{R}^{1}$ contains a carbon atom capable to form a stable radical, the direction of the fragmentation can vary from the one illustrated, yielding unwanted side-products.

Scheme 4. Huang's proposed mechanism.

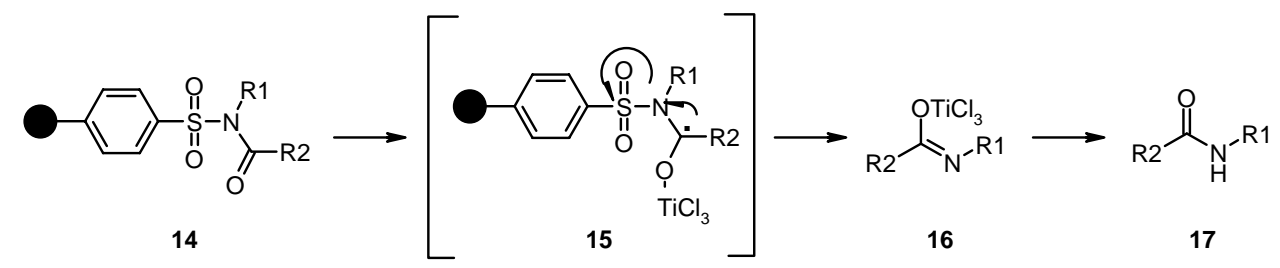

D'Herde and De Clercq [18] used the single-electron reductive property of samarium(II) for the cleavage of PS- $\beta$-benzolyloxysulfones in a Julia-Lythgoe olefination, where the SP-sulfone partly works as a traceless linker (Scheme 5). The best $\mathrm{E}: \mathrm{Z}$ ratio $(\mathbf{1 9 : 2 0}=94: 6)$ was obtained when $\mathrm{R}=\mathrm{Bz}$ and 1,3-dimethyl-3,4,5,6-tetrahydro-2(1H)-pyrimidinone (DMPU) was used as an additive, although isolated yields were rather low (around 25\%).

Scheme 5. De Clercq's proposed mechanism.

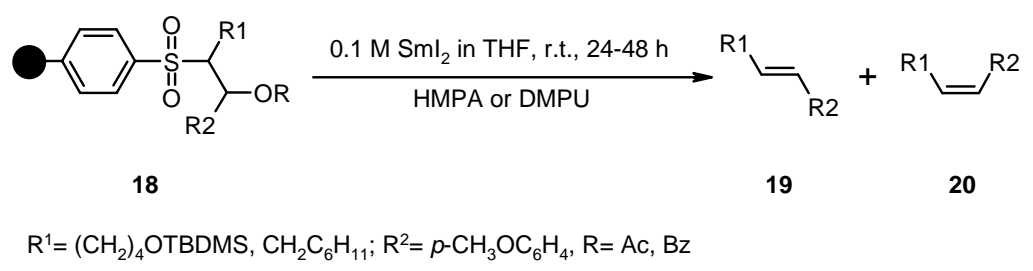

Radical cyclization to prepare azoles has been explored in solid-phase synthesis using a thiophenyl linker [19], using benzimidazole as a probe heterocycle. To prove the validity of the methodology, the same reactions were also carried out in solution (Scheme 6). Radical precursors 21a-c were loaded on the resins through the acid moiety on the arylthiol.

Scheme 6. Bowman's sulfur-based linker.

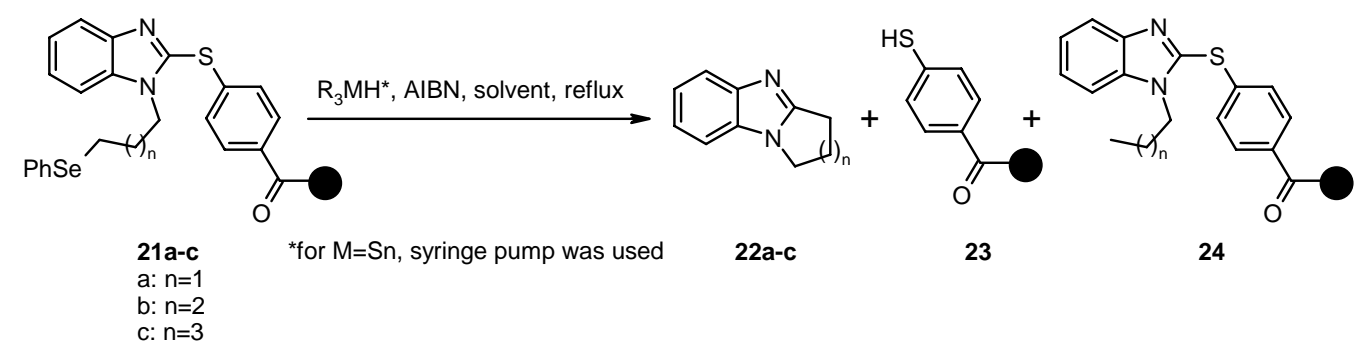


For $\mathrm{n}=1$, under the best conditions, only a $11 \%$ of desired product 22a was observed, in line with the results obtained in solution, indicating that this 5-exo-trig cyclization is not strongly favored. For $\mathrm{n}=2$ the reaction was smoother and use of TBTH gave a $60 \%$ of isolated $\mathbf{2 2} \mathbf{b}$. When TBGH was used, a lower yield of 22b was isolated (22\%), while TTMSS gave similar results (20\%). For $n=3$, again poor yields were obtained (4\%), just as the reaction run in solution with TBTH. Three resins were used for the scope, however, if Wang and Rink resins gave similar results, amino-Merrifield proved to be unsuitable, as only traces of desired products were found (for $n=1$ ). The rationale behind the use of 2thioaryl derivative is summarized in the proposed mechanism (Scheme 7).

Scheme 7. Bowman's proposed mechanism.

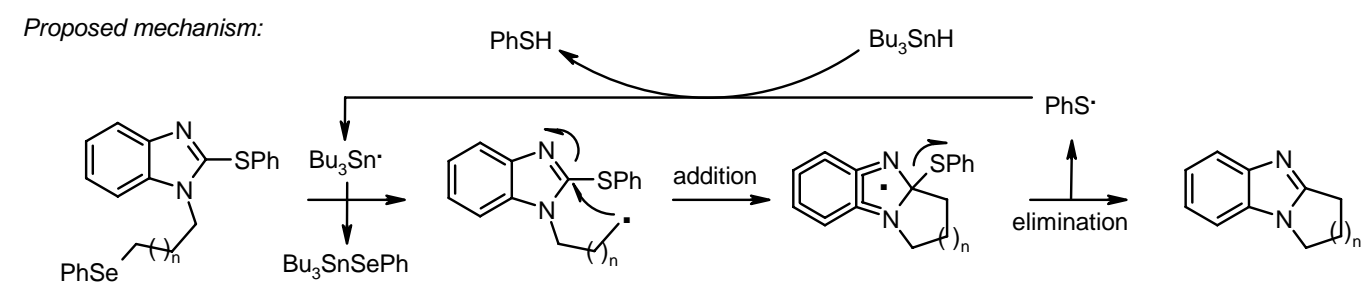

The 2-thiophenyl group behaves as a radical leaving group to achieve rearomatization and, subsequently, as radical carrier to abstract a hydrogen atom from the metal hydride, propagating the chain [20]. Following the mechanism, it is possible to note that the desired cyclized product 22 is released after formation, while side-products, such as non-cyclized, reduced $\mathbf{2 4}$ and unreacted starting material are left on the resin.

In order to shorten the reaction times, focused microwave irradiation was also used. As a test case radical precursor 21b $(n=2)$ on Wang resin was taken. Under optimized conditions (using AMBN) a $52 \%$ yield was obtained, very close to the $60 \%$ obtained under conventional heating, but with dramatically shortened reaction times $(20 \mathrm{~min} v s .7 \mathrm{~h})$. The reaction was also run on 21a $(\mathrm{n}=1)$ on amino-Merrifield resin, giving poor yields (3\% of 22$)$.

Among the new SP techniques, the $\alpha$-Hetero-Atom Substituted Carbonyl (HASC) traceless linker studied by Procter, occupies an important role. It exploits the SET characteristics of Sm(II) for reductive cleavage of an X-C bond. The general idea of the methodology starts with the attachment of $\alpha$-halo-carbonyl compounds to an appropriate resin, followed by further transformation of the resin-linked compounds, ending in the reductive cleavage from the resin (Scheme 8).

Scheme 8. Procter's HASC strategy.

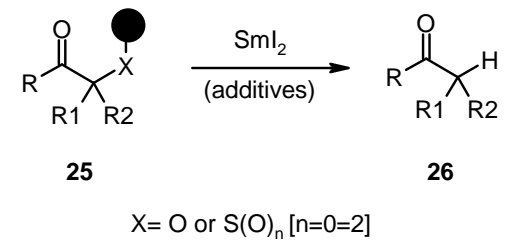

Heterocycle synthesis was performed following this strategy using a sulfur-linkage [21-23]. Benzylthiol resin 27 was prepared from Merrifield resin, treated with the appropriate $\alpha$-bromoacetamide and oxidized (Scheme 9). This activated substrate was then submitted to the 
Pummerer cyclization to yield oxindole 28, which could be cleaved using $\mathrm{SmI}_{2}$, giving the final products 29 in good yield, or alternatively, it could be further functionalized with allyl bromide prior to detachment (Scheme 10).

Scheme 9. Procter's use of a sulfur linker for the synthesis of oxindoles.

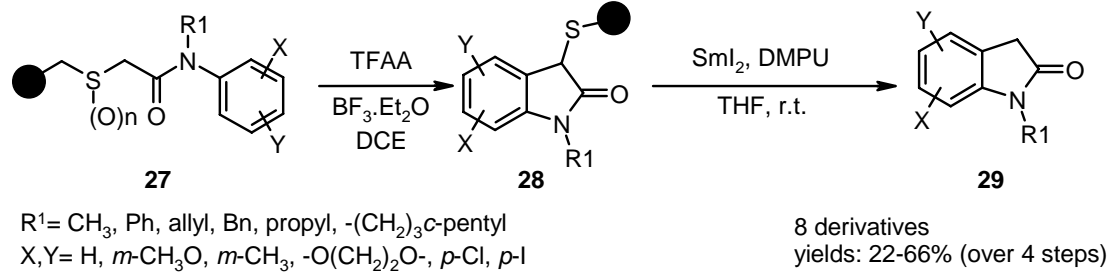

Scheme 10. Procter's synthesis of 3-allyl oxindoles.<smiles>COc1ccc2c(c1)N(Cc1ccccc1)C(=O)C2S(C)(=O)=O</smiles>

30

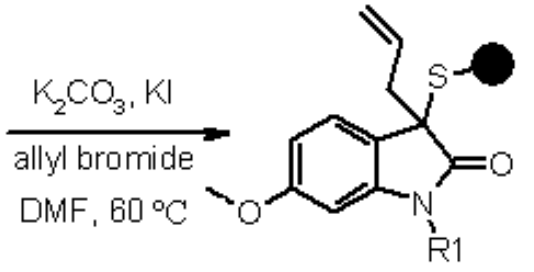

31

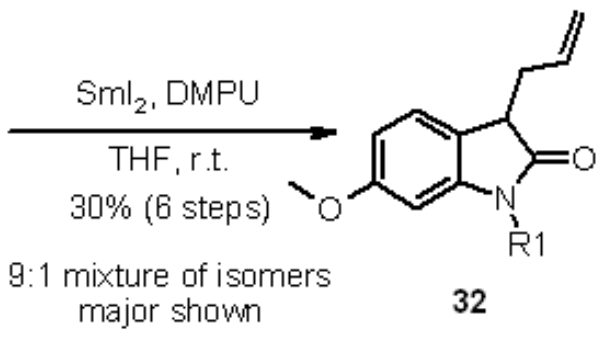

32

Tetrahydroquinolones were prepared in a similar fashion, using a Heck reaction followed by sulfur oxidation and Michael addition to perform cyclization. A couple of examples are shown in Scheme 11.

Scheme 11. Procter's use of a sulfur linker for the synthesis of tetrahydroquinolones.<smiles>[R]c1cc([R])c(N([R])C(=O)CSCC)c([X])c1</smiles>

33

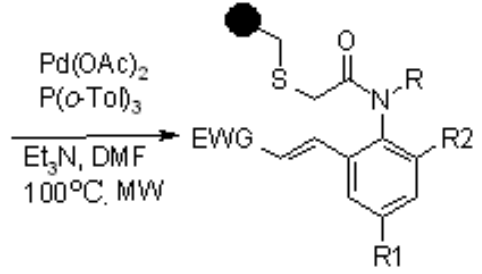

34
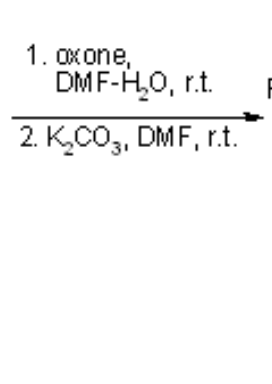

$\mathrm{R}=\mathrm{CH}_{3,} \mathrm{Bn} ; \mathrm{R}^{1}=\mathrm{R}^{2}=\mathrm{H}_{1} \mathrm{~F} ; \mathrm{R}^{1}=\dot{\mathrm{Pr}}, \mathrm{R}^{2}=\mathrm{H}$ $\mathrm{X}=\mathrm{Br}, \mathrm{l} ; \mathrm{EWG}=\mathrm{CO}_{2} \mathrm{t} \mathrm{EU}, \mathrm{C}(\mathrm{O})$ morpholine

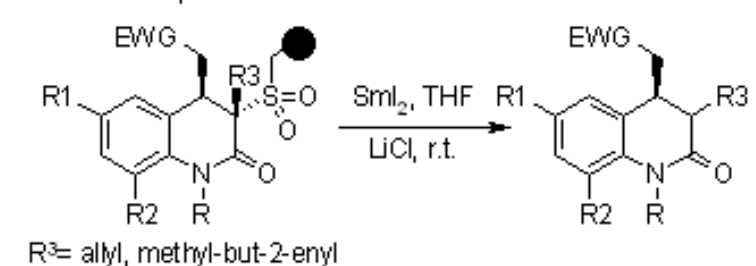

37
38

Reductive cleavage from the resin was performed before further functionalization to give $\mathbf{3 6}$ or the sulfur-bearing carbon atom was derivatized and only subsequently cleaved off the resin to give $\mathbf{3 8 .}$

In Scheme 12 an example of samarium iodide-mediated cyclative cleavage to give $\mathbf{4 0}$ through a radical or ionic process is shown. 
Scheme 12. Example of $\mathrm{SmI}_{2}$-mediated cyclative cleavage.

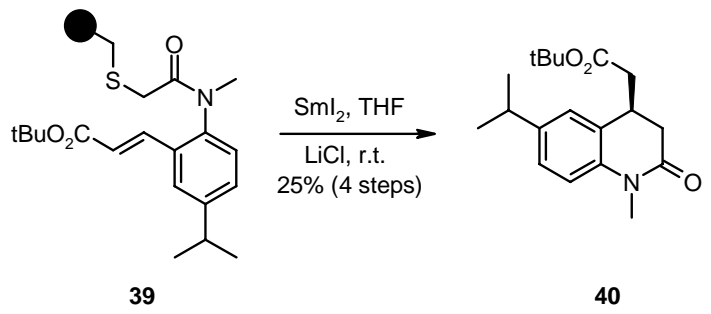

The general mechanism for samarium-induced reductive cleavage of $\alpha$-heteroatom carbonyl compounds is displayed in the scheme below (Scheme 13).

Scheme 13. Procter's proposed mechanism.

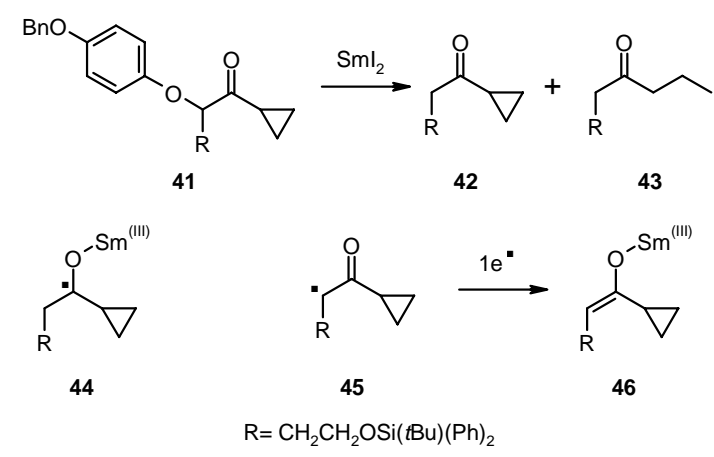

The cleavage mechanism was studied in solution for cyclopropyl derivative 41. The samarium SET reductive cleavage would account for a radical type 44, leading to cyclopropyl ring opening (compound 43) via a cyclopropylmethyl radical. However, since there was no trace of ring-opened derivative 43, the alternative and more probable explanation accounts for different intermediates such as 45-46. The presence of a samarium enolate of the kind of $\mathbf{4 6}$ was demonstrated by the capturing of electrophiles during cleavage.

\subsubsection{Selenium (and Tellurium)-based Linkers}

A number of reports deal with the use of selenium linkers, which are known to be amenable to several cleavage strategies, and the topic has been reviewed recently [24]. The following section is simply meant to highlight the value of such types of linkers in contemporary SPS.

Ruhland and coworkers produced interesting works replacing sulfur with selenium, which proved to be a versatile traceless linker in both aliphatic $\mathrm{C}-\mathrm{H}$ bond-forming reactions, taking advantage of easier homolytic cleavage, and alkene formation by selenium oxidation followed by $\beta$-elimination. In a first paper [25] bromopolystyrene 47 was obtained through thallium acetate-catalyzed bromination of commercially available polystyrene.

Scheme 14. Ruhland's synthesis of selenium-based resin.

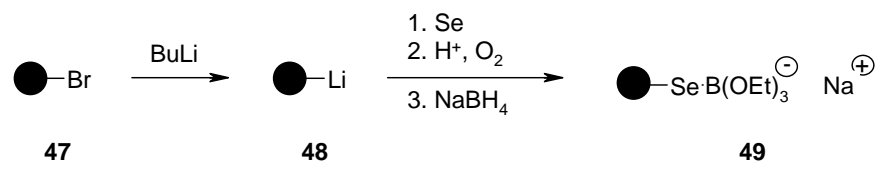


After lithiation with BuLi, the resin was treated with selenium, in order to replace the metal anion. Reduction with $\mathrm{NaBH}_{4}$ was necessary because of the occurrence, during work-up in the presence of air (step 2) of Se-Se bridges. Reduction gave back the swelling properties to the resin, lost because of the high degree of cross-linking encountered in step 2. Loading of resin $\mathbf{4 9}$ resulted lower compared to the bromopolystyrene 47 , probably due to incomplete reaction with selenium or partial availability of the metal in the resin itself. To prove the utility of resin 49, it was carried out the synthesis of an alkyl-aryl ether small library using the Mitsunobu reaction (Scheme 15).

Scheme 15. Ruhland's synthesis of ethers library.

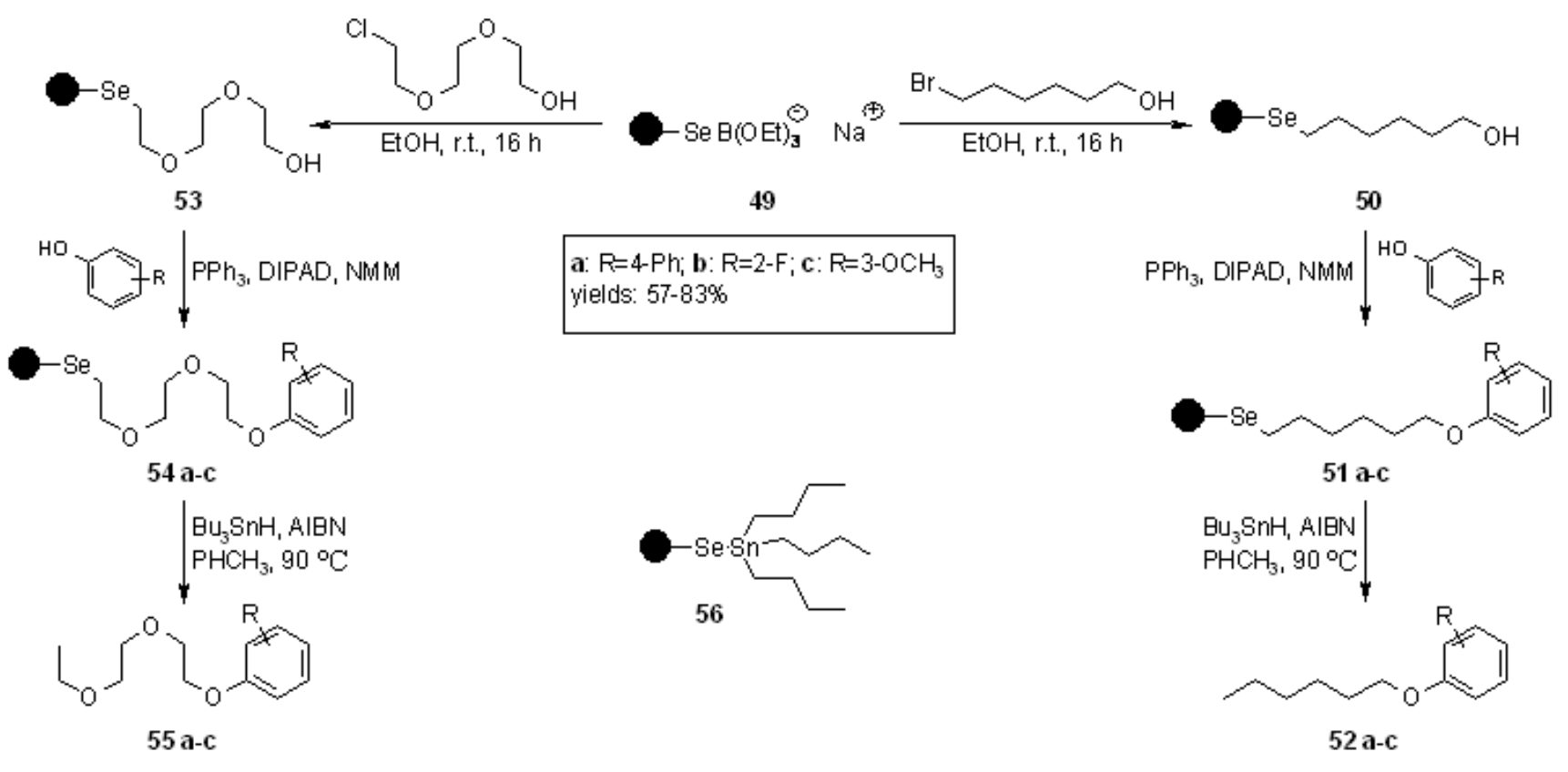

After application of the Mitsunobu protocol, cleavage was performed under radical conditions. Contrary to the reaction carried out in solution, the tributylstannyl phenyl selenide 56, often a problem for efficient purification, remained anchored on the SP and the only by-product of the cleavage was the excess $\mathrm{Bu}_{3} \mathrm{SnH}$, which was efficiently separated by SP extraction.

Polymer-bound tellurium was next introduced by the same group [26], which was prepared exploiting a strategy similar to the above. Also in this case a small library based on the Mitsunobu reaction was produced, following tributyltin-mediated product detachment. Interestingly and contrary to expectations, it was found that the reactivity of the polystyrene-bound tellurium towards homolysis is lower in comparison to the polystyrene-bound selenium. This is possibly due to residual elemental tellurium (remaining within the resin) interfering with the radical chain process in the cleavage step.

The selectivity of bond cleavage offers an important general advantage when utilizing this SP methodology. However, contamination of the cleaved products by tin derivatives can hold back its use, especially for biologically interesting libraries. To address this issue the same group showed later that it is possible to use greener traceless cleavage, exploiting silanes or germanes as valid alternatives to tin-containing reagents. In fact, it was demonstrated [27] that among others especially tris(trimethylsilyl)silane, performs similarly to tributyltin hydride in homolytically cleaving alkylselenium derivatives. On the other hand, resin-bound tellurium appears to have a preference for tris(trimethylsilyl)germane (see Figure 16). In general, however, both yield and purity are superior for 
the polystyrene-bound selenium, though tellurium is somewhat less sensitive to the number of cleavage reagent equivalents.

Figure 16. Tris(trimethylsilyl)germane.

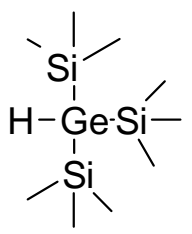

A chiral selenium electrophile has been also developed and applied to the stereoselective selenenylation of alkenes. The linker has been generated on solid-phase by loading a methoxymethyl-protected selenophenol to a suitable polymeric support, and subsequently generating the electrophile by reaction with bromine at low temperature (Scheme 17). Indeed, mesoporous silica proved superior to other supports, although its loading is usually low. Polystyrene works better than TentaGel and it was predominantly used for the stereoselective additions. Selenenylation of alkenes in the presence of a suitable nucleophile, usually an alcohol, yielded up to $80 \%$ ee, making the polymer-supported reagent almost equally attractive as its soluble counterpart (Scheme 18).

Scheme 17. Synthesis of asymmetric selenyl linker.<smiles>COCOC(C)c1cc(O)ccc1[Se]COC</smiles>

57<smiles>COCO[C@H](C)c1cc(OC)ccc1[Se]COC</smiles>

58<smiles>COCOC(C)c1cc(OC)ccc1Br</smiles>

59

Different cleavage protocols were shown in the paper, including the classical oxidation followed by $\beta$-elimination, tributyltin hydride-mediated homolytical carbon-selenium bond cleavage and also the use of allyltributyltin, which led to the introduction of an allyl moiety in the product (Scheme 18).

Scheme 18. Application of asymmetric selenyl linker.

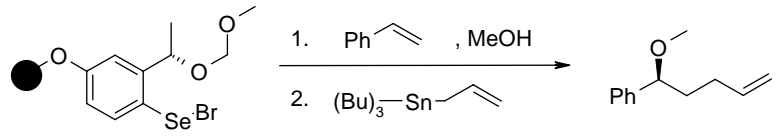

59

60

Supported selenyl bromide was prepared also by Nicolaou and coworkers [28] and showed its utility, beside that of a series of other selenium-containing resins, for the use in combinatorial chemistry as linkers (Scheme 19). 
Scheme 19. Nicolaou's preparation of PS-selenyl bromide.

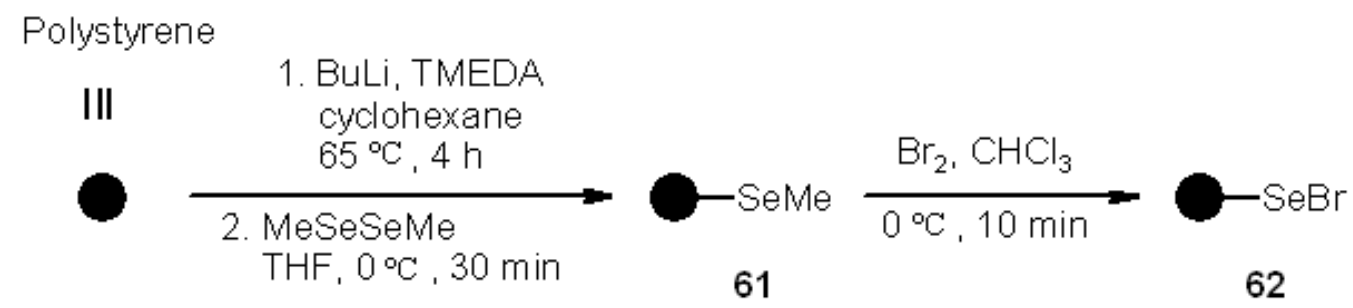

Compound 62 was exploited to load alkene 63, and subsequently cleaved under radical conditions (Scheme 20).

Scheme 20. Nicolaou's SP alkene selenylation.

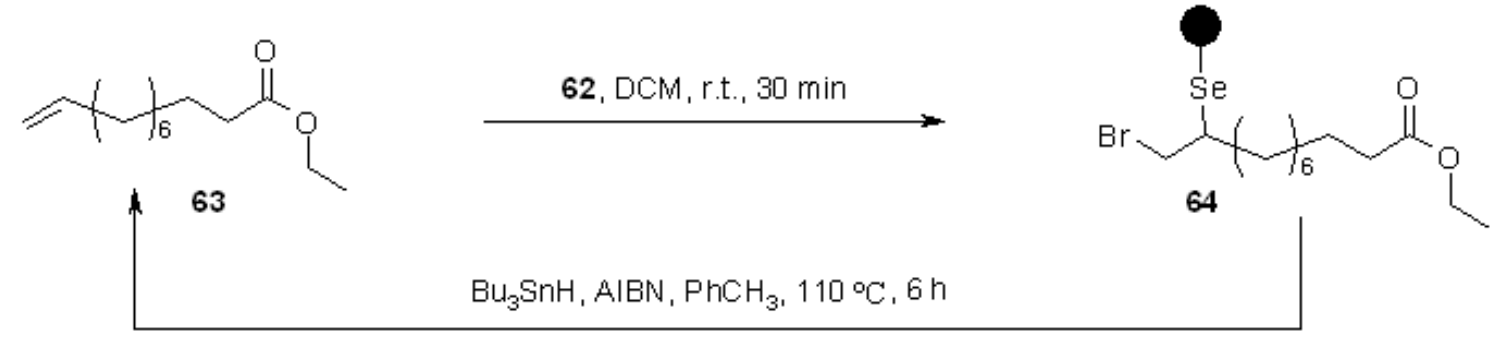

Compound 62 can also be reduced to resin 65 (Scheme 21), which can be reacted with halides to load the corresponding alkane. Standard cleavage under radical conditions gave reduction product 69 (Scheme 21).

Scheme 21. Nicolaou's modification of PS-selenyl bromide.

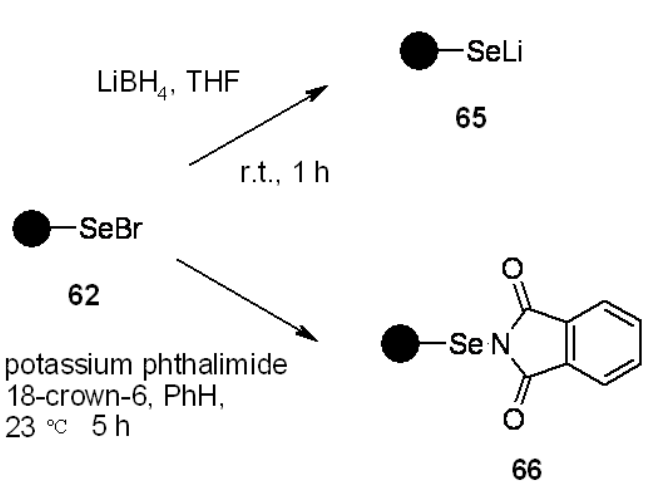

Scheme 22. Nicolaou's use of resin 65.

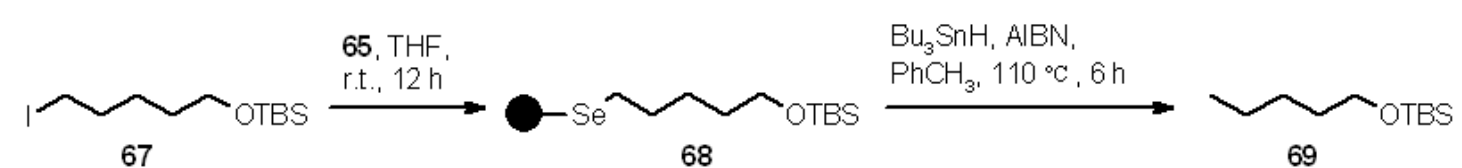

In addition, the same resin $\mathbf{6 2}$ can give the phtalimido-derivatives 66, used for insertion of benzyl alcohol (product 71) onto the alkene moiety (70), after radical cleavage (Scheme 23). 
Scheme 23. Nicolaou's use of resin 66.<smiles>BrOC[C@H]1OC=C[C@@H](OBr)[C@@H]1Br</smiles>

70

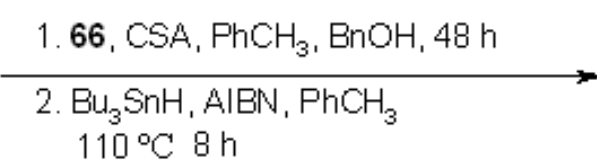

$110^{\circ} \mathrm{C} 8 \mathrm{~h}$<smiles>BrOC[C@H]1O[C@H](OBr)C[C@H](OBr)[C@@H]1Br</smiles>

71

In another interesting work, the same group [29,30] used selenium-based linkers, followed by reductive radical cleavage from the resin, for the synthesis of a small library of 2-deoxy glycosides (Scheme 24).

Scheme 24. Nicolaou's synthesis of 2-deoxy glycoside library.

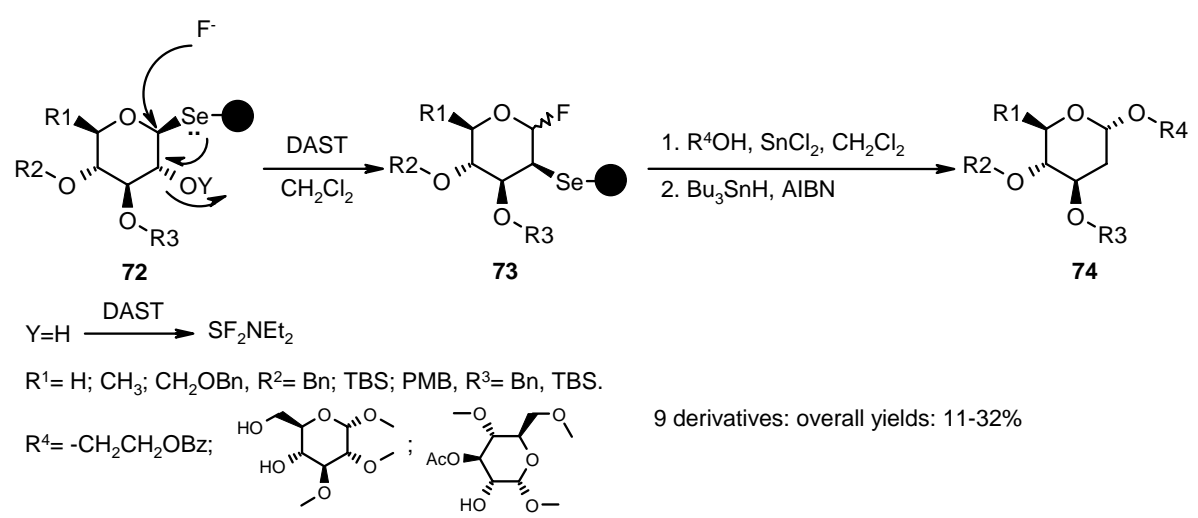

A small library of $3 \times 3$ was generated ( 3 "donors"-the sugars described by $\mathrm{R}^{1}, \mathrm{R}^{2}$ and $\mathrm{R}^{3}$ - and 3 "acceptors", described by $\mathrm{R}^{4}$ ), with overall yields between $11-32 \%$. The key reaction was the 1,2-seleno migration promoted by diethylaminosulfur trifluoride (DAST), while radical cleavage was typical of selenium-based linkers. Selenium-functionalized resins were exploited by Nicolaou's group [31,32] also in the studies towards the synthesis of heterocycles, namely indolines, indoline-based policyclic systems and indoles, which were obtained via different mechanisms of radical cleavage (Scheme 25).

Scheme 25. Nicolaou's SP selenium-based synthesis of heterocycles.

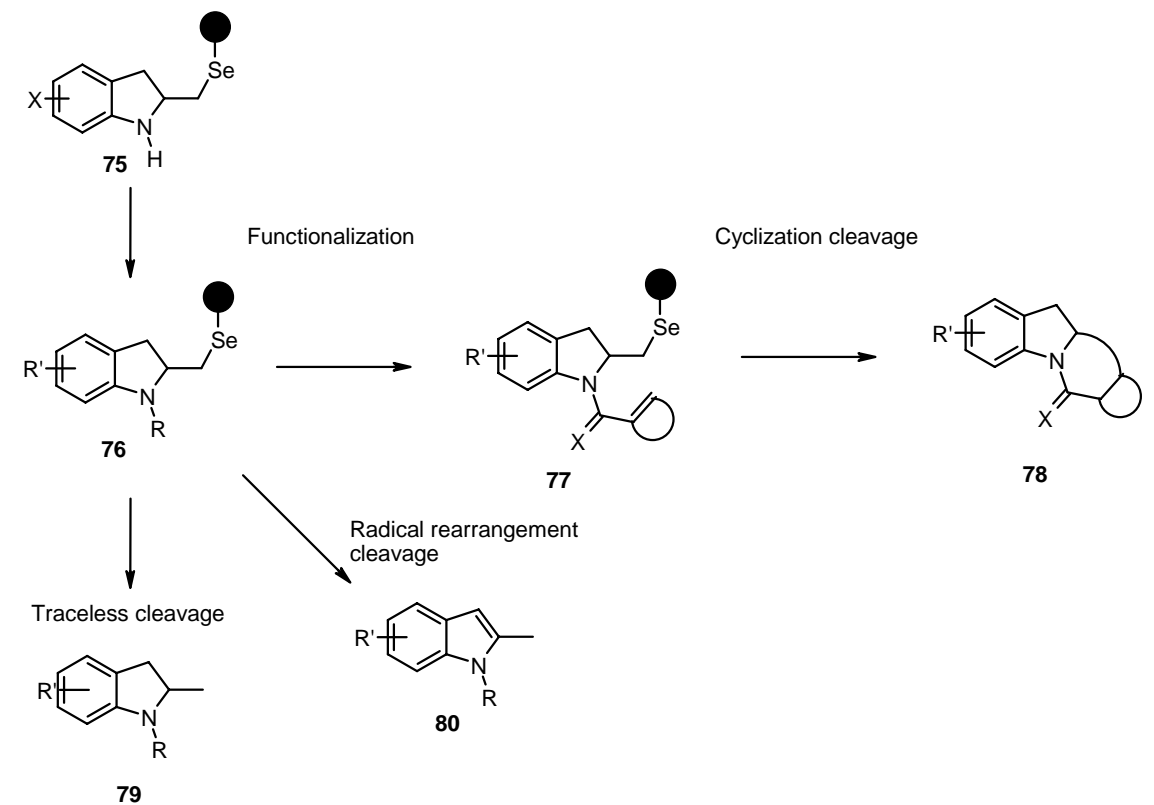


Starting from solid-supported selenyl bromide, indolines $\mathbf{7 5}$ were prepared by Lewis-acid mediated cycloloaddition using the appropriate $o$-allyl anilines. Subsequent functionalization at the nitrogen atom and/or at the substituent on the benzene ring allowed the introduction of additional diversity elements. Use of different cleavage strategies enabled then the preparation of small libraries of drug-like molecules. This represents an interesting extension of this methodology to combinatorial chemistry for the synthesis of bioactive compounds. In Scheme 26 a classical traceless cleavage is shown. The mechanism entails the homolytic cleavage of the Se-C bond, therefore generating a carbon-centred radical, quenched by the hydrogen-donor $\mathrm{Bu}_{3} \mathrm{SnH}$.

Scheme 26. Nicolaou's selenium traceless linker strategy.

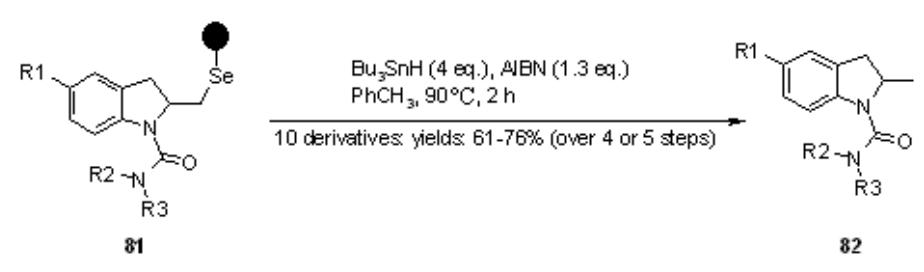

In the cyclization cleavage shown in Scheme 27, formation of a carbon-centred radical was exploited by using an appropriate alkene moiety on the nitrogen side-chain. Radical addition/cyclization was then followed by quenching of the resulting radical by tin hydride. The products of the reactions were polycyclic indoline derivatives, with only one diasteroisomer isolated.

Scheme 27. Nicolaou's synthesis of tetracycle 84.
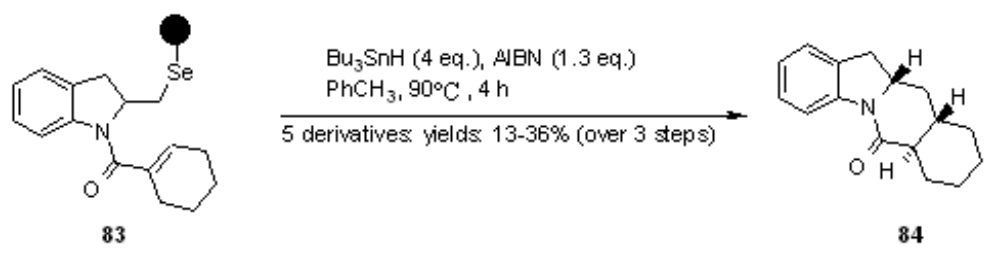

An example of cleavage by radical rearrangement is shown in Scheme 28. In this case, the lack of $H$-donor makes the $C$-centred radical enough long-living to perform a shift. Although the nature of the shift is unknown, any of the supposed intermediates radical $\mathbf{8 8}$ and $\mathbf{8 9}$ can undergo oxidation to the indole ring by loss of a hydrogen atom.

Scheme 28. Nicolaou's $C$-centred radical shift.

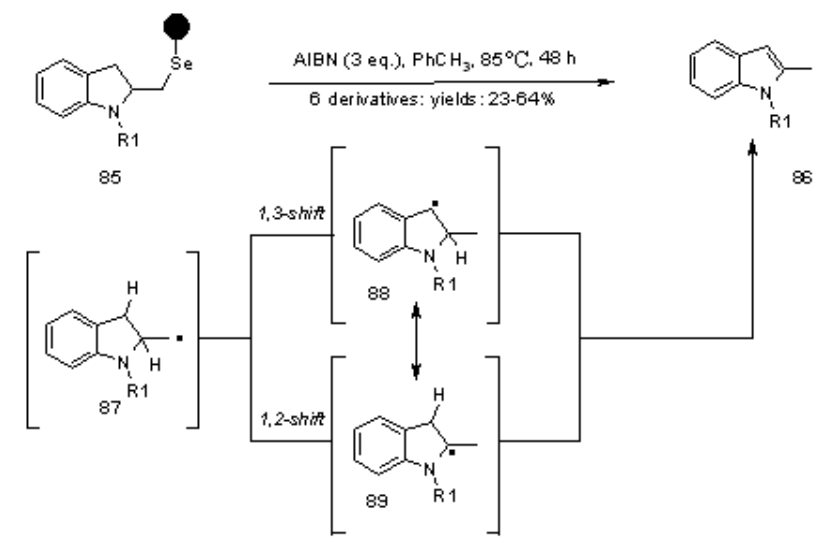


Very interesting are also other examples from the same group [33], which used the same cycloloadition/radical cleavage methodology to construct [3.3.1]bicycles from properly designed substrates (Schemes 29 and 30).

Scheme 29. Nicolaou's synthesis of bicycle 91.

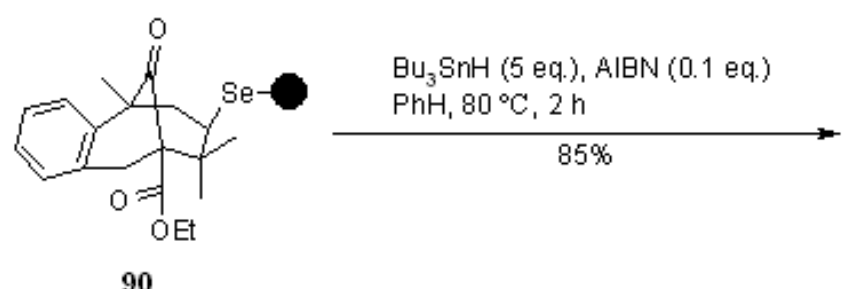

90

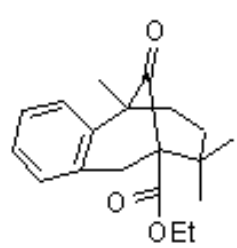

91

Scheme 30. Nicolaou's synthesis of bicycle 93.

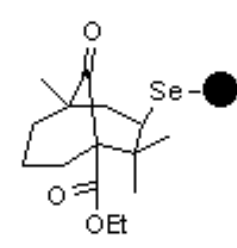

92

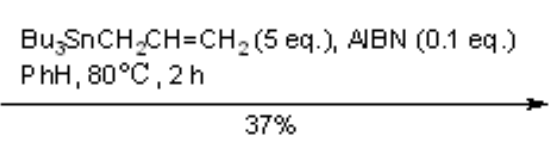

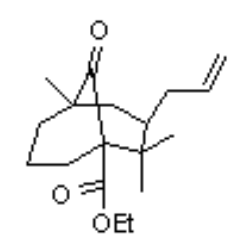

93

As before, bicycles 90 (Scheme 29) and 92 (Scheme 30) were prepared by electrophilic cyclization with a solid-supported-SeBr resin, while cleavage was performed in a traceless manner (Scheme 29) or by trapping the resulting $C$-centred radical with allystannane (Scheme 30 ).

Although the methodologies shown above proved efficient for the synthesis of small compound libraries, it is important to highlight a major drawback that is implicit in the cleavage mechanism. In fact it was necessary to separate the desired products from the residues of the radical reaction, an option often tedious and difficult that biases the use of toxic tin derivatives for in-solution radical chemistry.

Another interesting cleavage methodology was reported by Engman and coworkers [34], who studied the radical carbonylation/cyclization to make tetrahydrofuran-3-ones. The method was first developed in solution, exploiting alkyl phenyl selenides and then applied to a selenium-based resin 94, prepared in a few steps from cross-linked (1\%) polystyrene (Scheme 31).

Scheme 31. Engman's use of a selenium linker.

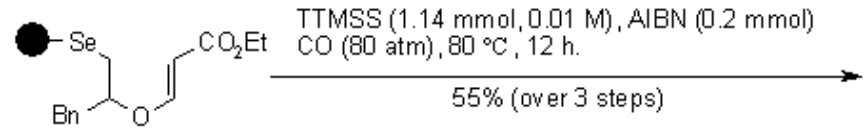

94

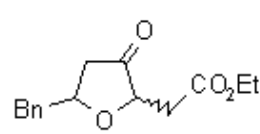

95

TTMSS was chosen instead of the more common TBTH, which gave a large amount of reduced (non-cyclized) product, whereas, increasing the concentration of the H-donor resulted in poorer yields of the desired vs. reduction product. TBGH) gave worse results compared to TTMSS. Lower carbon monoxide pressure gave also more product of reduction. The final product 95 was isolated as a 9:1 mixture of cis/trans isomers (55\% yield calculated over 3 steps, 2 of them for the synthesis of 94 ). 
Fujita et al. [35] used PS-arylselenides for the selenolactonization on 3-butenoic acids with successive cleavage via radical reduction (Scheme 32). The yield of the process to give $\mathbf{9 8}$ was rather low (20\%), a result explained by $\mathrm{Ph}_{3} \mathrm{SnH}$-promoted side-reactions with the amide linker.

Scheme 32. Fujita's SP selenolactonization of 3-butenoic acids.

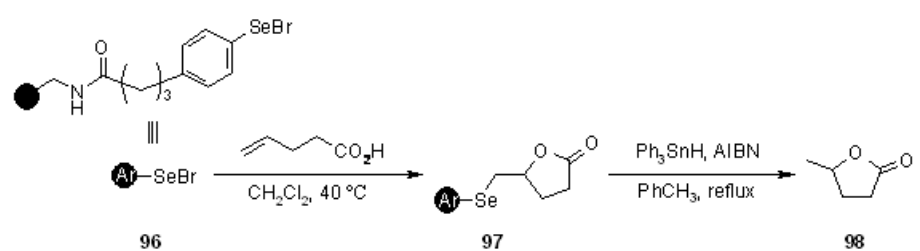

Polystyrene-supported selenosulfonates can be conveniently prepared from the easily accessible selenobromide by reaction with arylsulfinates. Qian and Huang [36] studied the radical addition of such PS-selenosulfonates to alkenes, with subsequent oxidation-elimination of selenium to obtain vinyl sulfones (Scheme 33).

Scheme 33. Huang's synthesis of selenosulfonates.

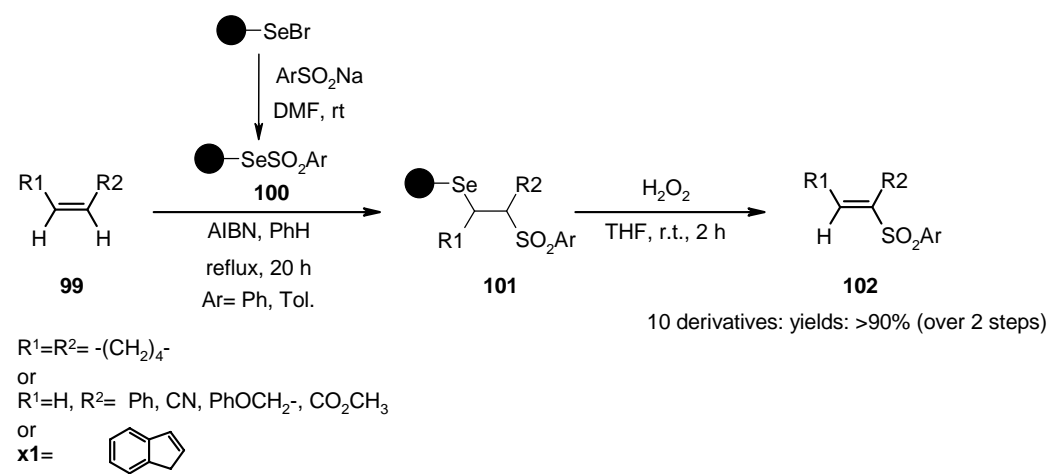

Radical addition was performed under classical radical conditions and the overall yields after cleavage were rather high. The same group also performed the radical addition of PSarylselenosulfonates to alkynes, followed by oxidation to selenoxide and syn-elimination to give acetylenic sulfones 105 [37] or by acidic hydrolytic cleavage from the SP, for the preparation of $\beta$-keto sulfones 106 [38] (Scheme 34).

Scheme 34. Huang's synthesis of acetylenic sulfones and $\beta$-keto sulfones.

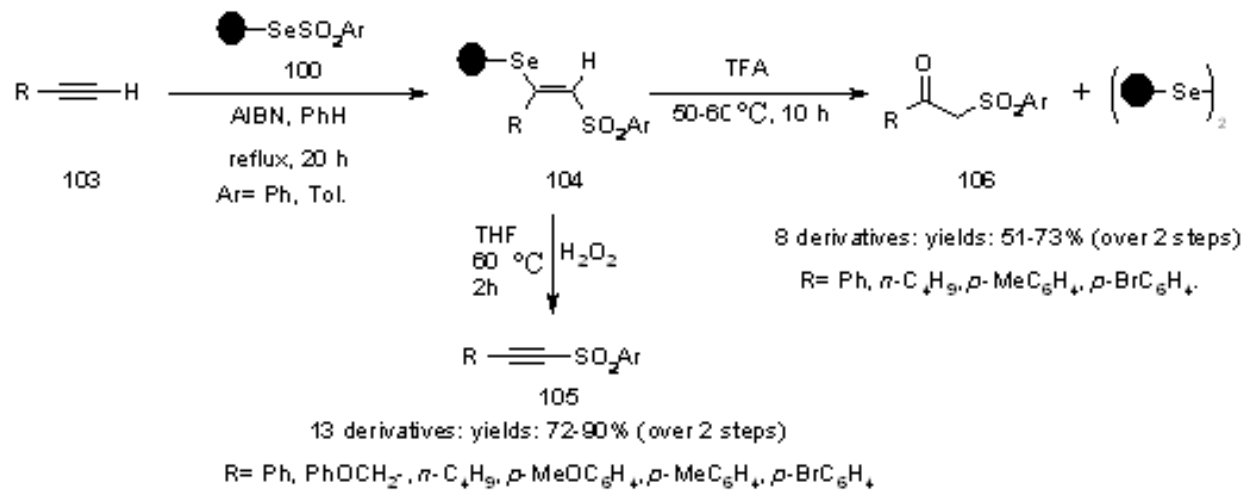


Cleavage under oxidation-elimination conditions (to give 105) gave higher yields compared to the acidic cleavage (to give 106), as shown. In any case, there was practically no need for further purification after cleavage from the resin and the PS-diselenide obtained after acidic cleavage could be regenerated.

In another interesting work, a solid-supported selenosulfone as radical transfer agent [39]. In this case however, the reaction led to a cyclization product that, therefore, remained anchored to the SP. Only successively this was cleaved form the resin by the classical oxidation-elimination protocol (Scheme 35).

Scheme 35. Huang's SP-atom transfer cyclization.

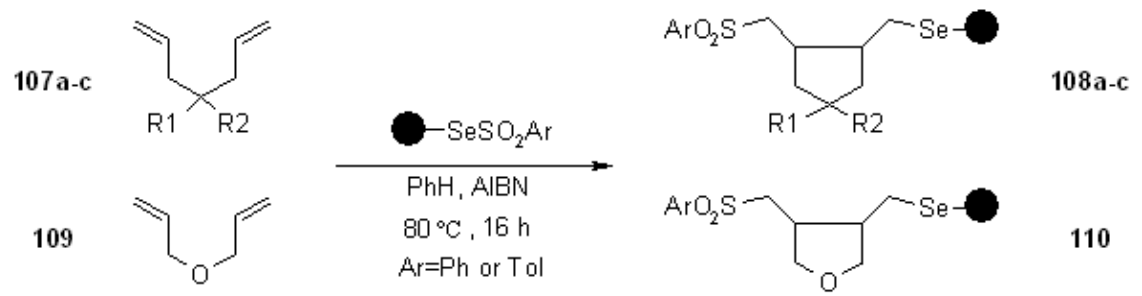

The isolated yields were calculated after cleavage (44-58\%, 12 examples), using two different selenylarylsulfonyl resins.

\subsubsection{Oxygen-based Linkers}

Samarium (II) iodide is a useful reagent, exploited for several scopes, having the characteristic of being a powerful single-electron reducing agent. All the examples below, where fission of either a carbon-oxygen or a nitrogen-oxygen bond has been exploited to release organic molecules from a solid support, make use of samarium iodide.

\subsubsection{Cleavage of Hydroxylamine-based Linkers}

Abell and colleagues [40] used $\mathrm{SmI}_{2}$ for the traceless cleavage of $\mathrm{N}-\mathrm{O}$ bond on substrates appropriately linked to SP. With this methodology it was generated a small library of amides and ureas (Scheme 36).

Scheme 36. Abell's amides and ureas library.

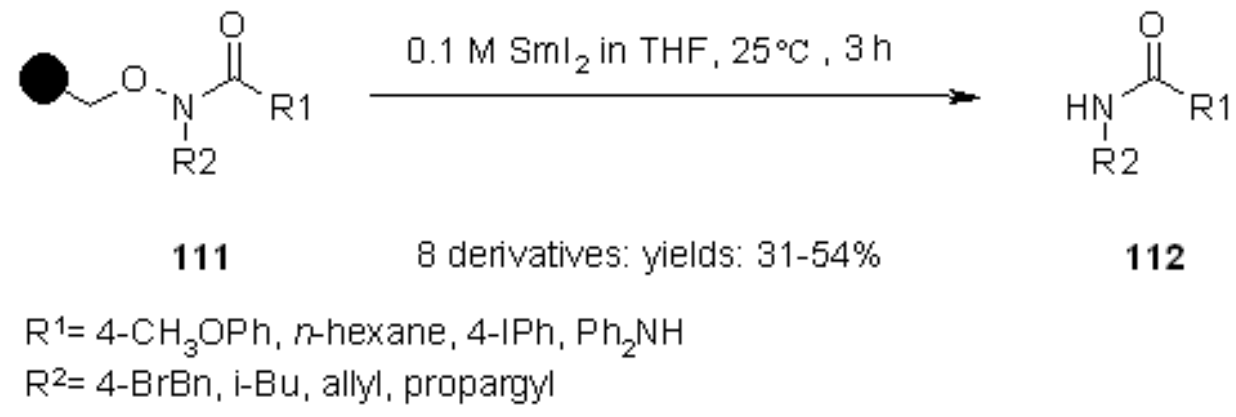

Although yields were modest, the purities of the cleaved products 112 were rather high.

The same cleavage technique was exploited by Andersson [41] that started from a polystyrene-based resin to synthesize tertiary amines (Scheme 37). 
Scheme 37. Andersson's reductive cleavage.

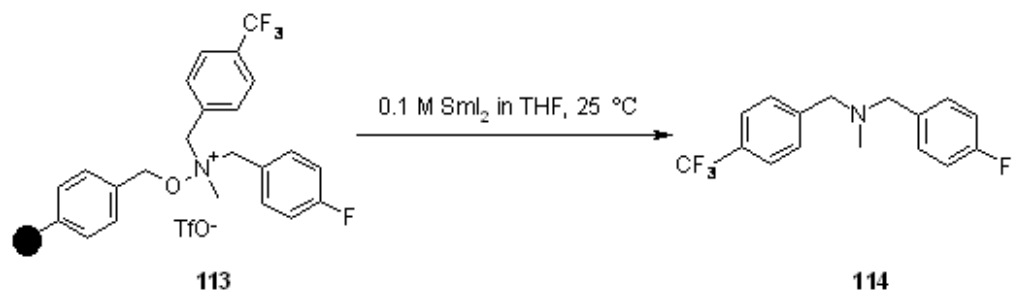

Although $\mathrm{SmI}_{2}$ gave clean cleaved products, the cleavage method used for the library production exploited the alternative LiI, which worked under neutral conditions.

Another example of the reductive cleavage promoted by $\mathrm{SmI}_{2}$ was shown by Meloni and Taddei [42], who applied it to the synthesis of $\beta$-lactams (Scheme 38). Polystryerene-bound hydroxylamine was once again used as the starting material.

Scheme 38. Taddei's synthesis of $\beta$-lactams.

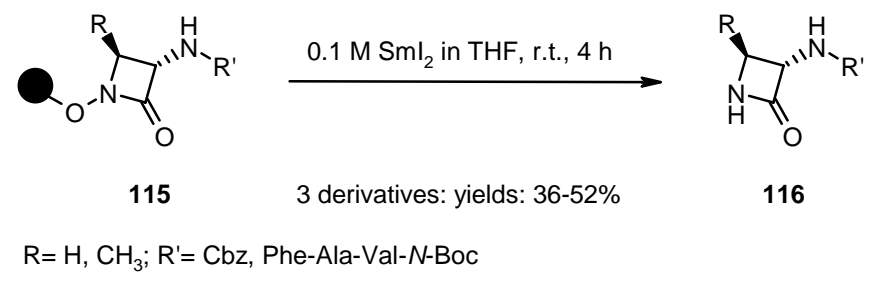

\subsubsection{Addition of Ketyl Radicals to $\alpha-\beta$ Unsaturated Esters}

Procter's group $[43,44]$ used a Sm(II) based radical chemistry for the enantioselective synthesis of $\gamma$-butyrolactones on SP exploiting $(1 R, 2 S)$-ephedrine linker as the chiral auxiliary. Bromo-Wang resin was first functionalized with $(1 R, 2 S)$-ephedrine and successively esterified at the alcohol group with the appropriate $\alpha, \beta$-unsaturated acyl chloride to give 117. This was, in turn, used in a reaction involving a ketyl radical addition to the anchored $\alpha, \beta$-unsaturated ester. The subsequent cyclative cleavage led to the formation of lactone $\mathbf{1 1 9}$ (Scheme 39).

Scheme 39. Procter's asymmetric lactones synthesis.

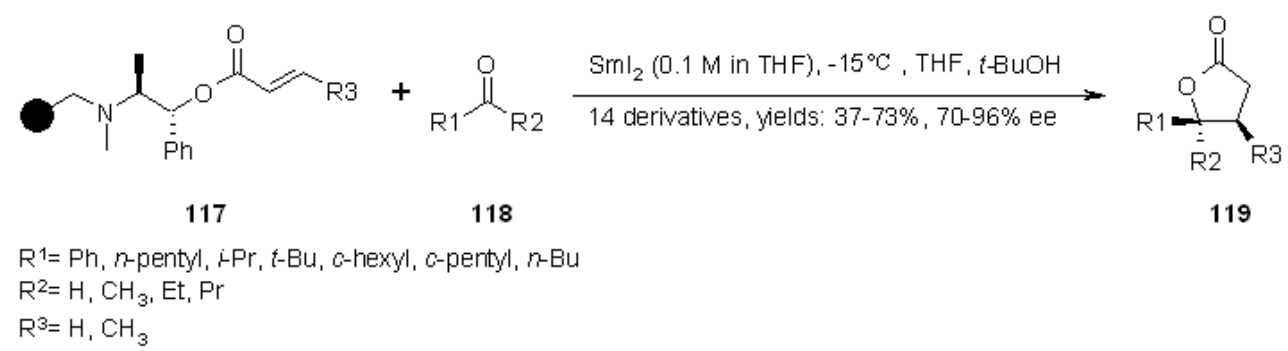

Under optimized conditions, the reaction gave rather high isolated yields (apart for a few exceptions) and diasteroselectivities. The same conditions were applied to the synthesis of lactone $\mathbf{1 2 1}$ (Scheme 40), a moderate DNA-binding metabolite isolated from Streptomyces GT61115. 
Scheme 40. Synthesis of lactone 121.

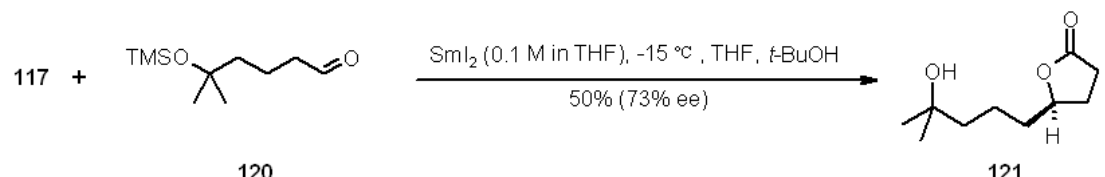

120

121

Cyclative cleavage gives back, beside the desired product, the resin functionalized with the asymmetric linker, which can be reused. Following Procter's lead on asymmetric induction for samarium-mediated radical synthesis of $\gamma$-butyrolactones, Dai and coworkers [45] linked axially chiral crotonates on Rink amide resin for the same purpose (Scheme 41).

Scheme 41. Dai's chiral crotonates on Rink amide resin.

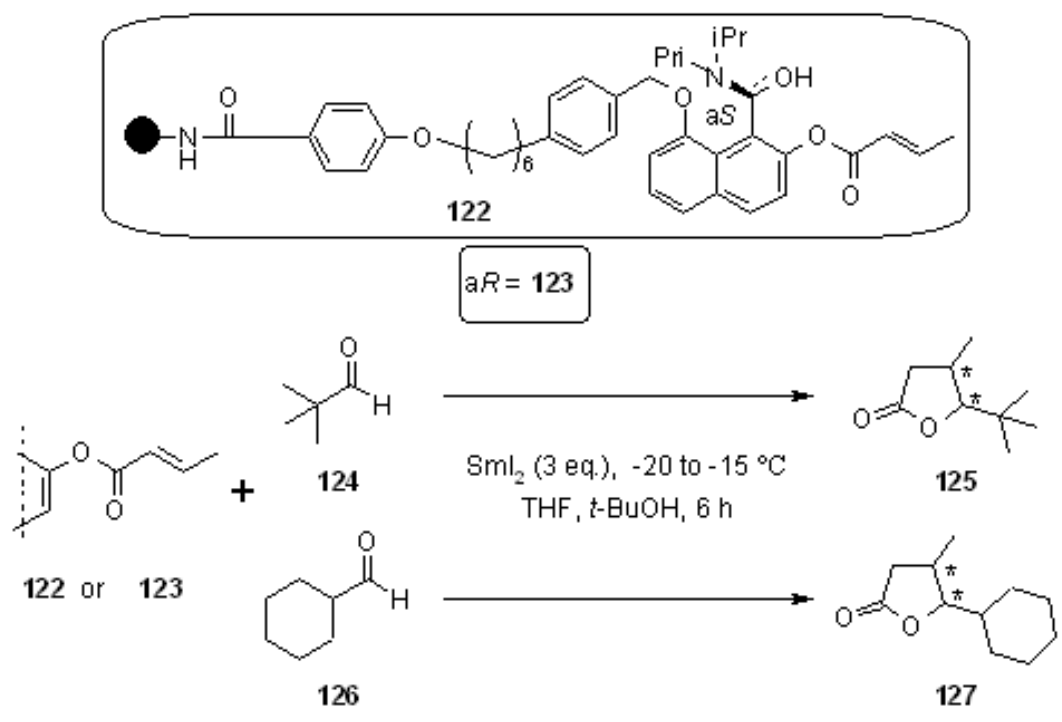

Reaction of 122 or 123 with aldehydes 124 and 126 gave reasonable isolated yields $(\mathbf{1 2 5}=58 \%$, $127=66 \%)$, with a good cis/trans selectivity $(\mathbf{1 2 5}=71: 29 ; \mathbf{1 2 7}=89: 11)$ and good ee $(\mathbf{1 2 5}-$ cis: $94 \%$, 125-trans: $89 \%$; 127-cis $=127$-trans: $88 \%$ ). The mechanism of chiral transfer was supposed to pass through an intermediate were the samarium atom coordinates the oxygens from 122 (or 123) and the oxygen of the ketyl radical which performs the 1,4-addition to the crotonates.

\subsubsection{HASC Traceless Linker}

The HASC traceless linker developed by Procter and mentioned above (see Scheme 8) has been exploited also using oxygen-based linkers. Applications of the ether linkage involve the synthesis of functionalized carbonyl compounds [46,47] (Scheme 42). Resin 128 was synthesized starting from bromo Wang resin, derivatized with the appropriate phenol and functionalised with $\alpha$-bromo- $\gamma$ butyrolactone. Lactone ring-opening was performed with some amines (pyrrolidine, morpholine, tetrahydroisoquinoline and methoxymethylamine) and the resulting alcohol protected accordingly. When Weinreib hydroxylamine was used, the obtained tertiary amide was successively reacted with a Grignard reagent $(\mathrm{BnMgCl}, i$-PrMgCl, $c-\mathrm{PrMgCl})$ to yield the corresponding ketone. Cleavage of 129 from the resin gave products $\mathbf{1 3 0}$ in good yields after 4-5 steps. 
Scheme 42. Procter's application of HASC strategy.

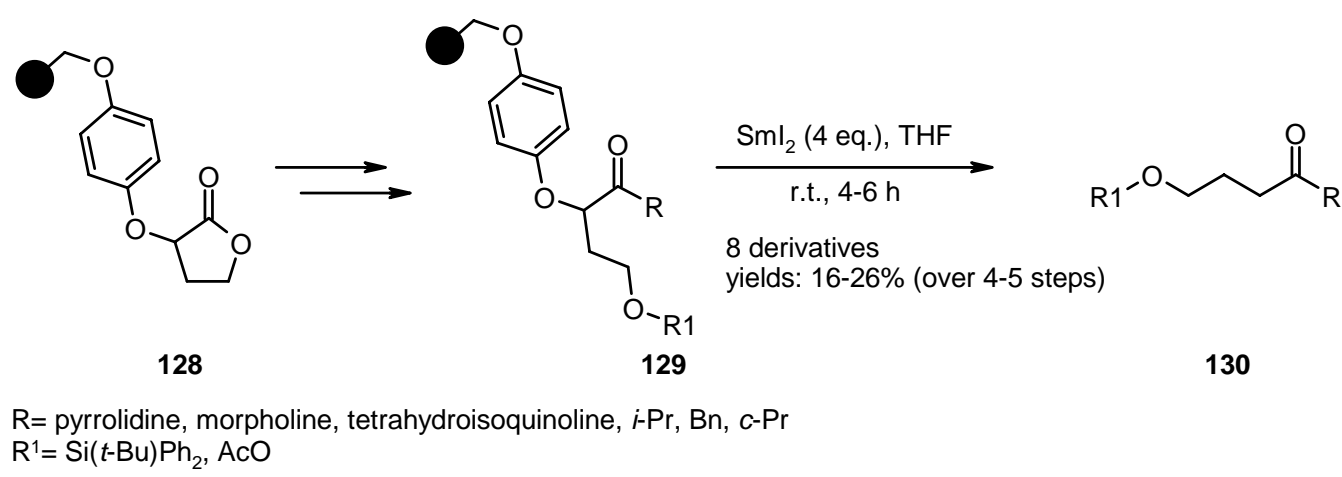

\subsection{4. "Carbon-Based” Linkers}

Floreancig's group $[48,49]$ used an oxidative release (often cyclorelease) on the appropriate polymersupported precursors. The mechanism involved in the oxidative cleavage is presented in Scheme 43.

Scheme 43. Floreancig's proposed mechanism.

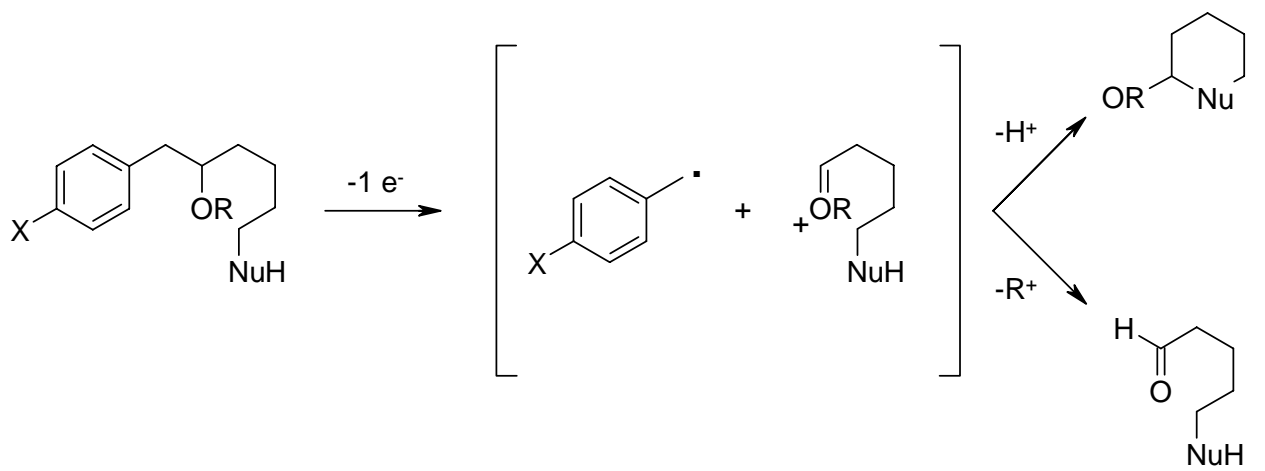

The process, (Electron Transfer Initiated Cyclization, ETIC) starts with single electron oxidation and mesolytic cleavage of the $C-C$ bond of the homobenzylic ether, forming a benzylic radical and an oxocarbenium ion. The resulting cation is successively transformed in a neutral species, giving a cyclised product if an appropriate nucleophile is present in the molecule, or an aldehyde (or ketone) if there is loss of the oxygen-bound " $\mathrm{R}$ " group (when present). To test this methodology on support, appropriate soluble ROMP polymers were prepared (Scheme 44).

The five equations prove the feasibility of the method, either by intramolecular trapping (equations 1-3), or by oxidative traceless release (equations 4-5). In the former case either formation of a $C-O$ bond (equation 1 ) or a $C-C$ bond (equation 2 and 3 ), which occurred with high diasteroselectivity, is shown. In the latter case formation of the aldehyde 138 of equation 4 requires loss of a THP group. 
Scheme 44. Application of IROMP polymers.

1)

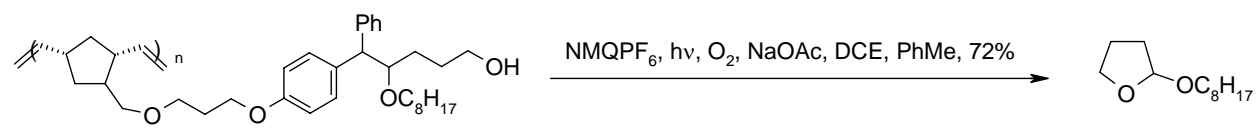

131

132

2)

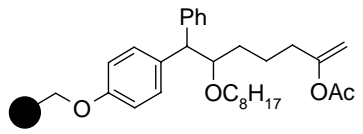

CAN, $\mathrm{NaHCO}_{3}$, DCE, $\mathrm{CH}_{3} \mathrm{CN}, 85 \%$

133

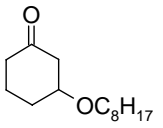

134

3)

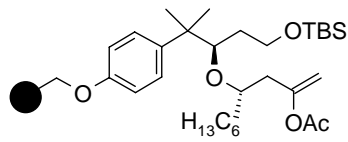

CAN, $\mathrm{NaHCO}_{3}$, DCE, $\mathrm{CH}_{3} \mathrm{CN}, 41 \%$

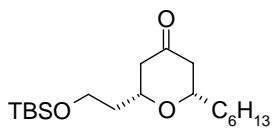

135

136

4)

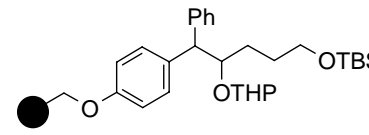

$\mathrm{NMQPF}_{6}, \mathrm{~h} v, \mathrm{O}_{2}, \mathrm{NaOAc}, \mathrm{DCE}, \mathrm{PhMe}, 78 \%$<smiles>O=CCCC[O+][Sb]</smiles>

137

138

5)

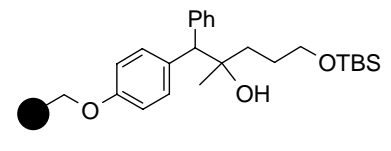

$\mathrm{NMQPF}_{6}, \mathrm{~h} v, \mathrm{O}_{2}, \mathrm{NaOAc}, \mathrm{DCE}, \mathrm{PhMe}, 89 \%$

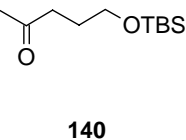

The purity of the products after this particular cleavage was rather high, due to the characteristic mechanism of the reaction, which ensured cyclocleavage or oxidative release only when the appropriate nucleophile was correctly installed in the molecule. This means that any impurity will be retained on the support, in a kind of purification step. Therefore, it is possible to perform several steps on the support, without the fear of accumulating side-products that have to be separated at the end of the process.

An interesting application of thiohydroxamate (THA) linker strategy was proposed by Routledge [50]. In previous reports [51,52] the same group presented a traceless THA linker, cleavable under photolytic conditions, but in the need of novel SP-linkers, a second-generation THA linker, stable under diverse chemical and photolytic conditions, was studied (Scheme 45).

Scheme 45. Routledge's THA linkeras polymer-supported radical source.

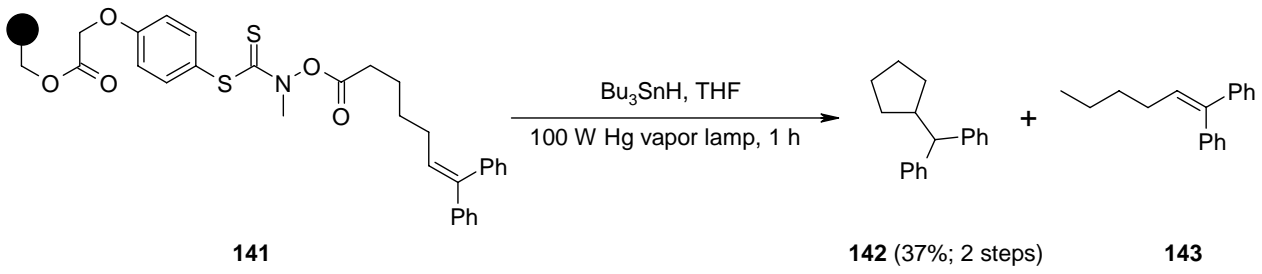

Although 141 proved to be stable to heat and light, it was sensitive to nucleophilic cleavage, which might make it of limited application for SP preparation of libraries. On the other hand, $\mathbf{1 4 1}$ was found useful as polymer-supported radical source. The nature of the linker allows the generation of a 
carbon-centred radical ultimately arising from a carbon-carbon bond fission, that can be appropriately exploited for successive reaction such as the one depicted above. The only product found was 142, even if isolated in moderate yield.

\subsection{Solid-Supported Reagents}

Another important aspect of radicals exploiting SP deals with the use of supported reagents for radical reactions. This combination allows toxic reagents, often difficult to separate from the desired products in solution, to be used with ease of purification and little contamination. Since the most important reagents in radical chemistry are represented by organostannanes, it was obvious that the initial efforts to translate in-solution radical chemistry to SP, would have involved tin-base reagents.

From this point of view, several works have tried to bind organostannanes on solid supports, in order to reproduce common radical reactions and make work-up easier with little or no contamination from the toxic metal. Within this class, organostannylhydrides occupy a dominant role. Neumann's group successfully immobilized dibutyltinhydride on SP with a two-carbon spacer between the metal and the aromatic ring of polystyrene to obtain resin 145, whose applications were highlighted in successive publications [53,54]. When employed for radical cyclization (Scheme 46), the results obtained were comparable with the same reaction run in solution with $\mathrm{Bu}_{3} \mathrm{SnH}(36 \%: 0 \%: 64 \%)$.

Scheme 46. Use of resin 145 for radical cyclization.

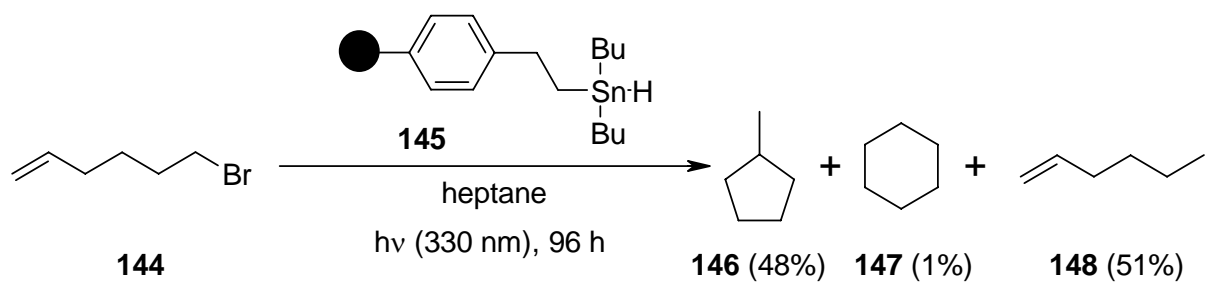

An application to the Barton dehydroxylation reaction [55], which has been exploited extensively later by the same group in a series of derivatized alcohols [56], is shown in Scheme 47. An example of deamination following preparation of the correspondent isocyanides is instead shown in Scheme 48.

Scheme 47. Barton dehydroxylation using resin 145.

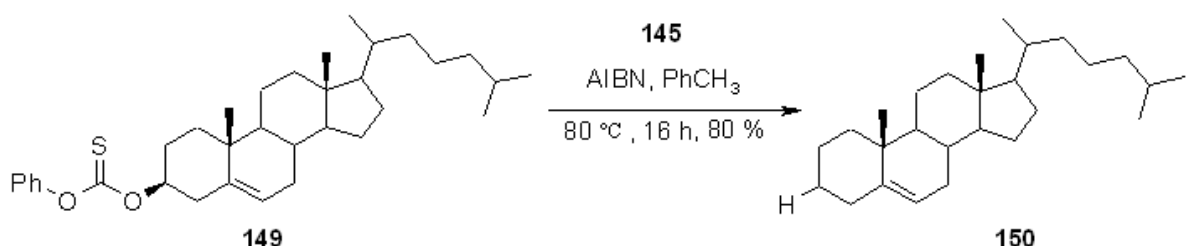

Scheme 48. Deamination reaction using resin 145.

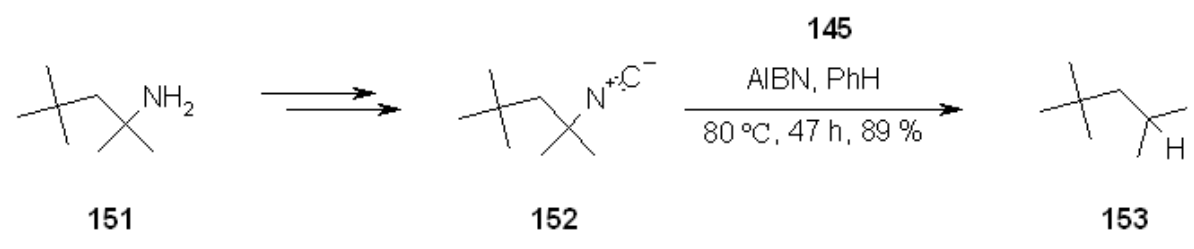


The supported tin hydride was recycled by reduction of the filtered resin, maintaining a good efficiency. Following a similar path, Dumartin and coworkers [57,58] synthesized supported organostannanes with spacer of 2, 3 and 4 carbon atoms, in order to avoid $\beta$-elimination of the stannane as by-product, therefore producing recyclable and more stable reagents for radical reactions. In a successive paper [59], the same group used the anchored $-\mathrm{Bu}_{2} \mathrm{SnX}(\mathrm{X}=$ halogen $)$ with a $\mathrm{C}-4$ spacer for catalytic reductions of haloalkanes in the presence of $\mathrm{NaBH}_{4}$ and AIBN. The length of the spacer proved to be important for reaction yields (due to more accessibility of the metal centre) and residual pollution by the metal.

Deleuze [60] prepared a macroporous organotin chloride by inclusion of a suitable organostannylstyrene precursor in the polymerization reaction. The so prepared supported reagent (in combination with $\mathrm{NaBH}_{4}$ ) proved to be comparable to $\mathrm{Bu}_{3} \mathrm{SnH}$ in reducing alkylhalides in solution.

Dumartin's group [61] investigated the possibility to use tin-based reducing agent in catalytic amount, as it had been done in solution [62]. In particular, their attention focused on the use of siloxanes, particularly polymethylhydrosiloxane (PMHS), for the regeneration of catalytic amounts of TBTH. However, PMHS proved ineffective using polymer-bound organostannane 154, evidently because it was incapable of penetrating the PS beads.

Scheme 49. Dumartin's catalytic approach.

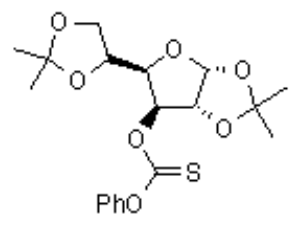

155

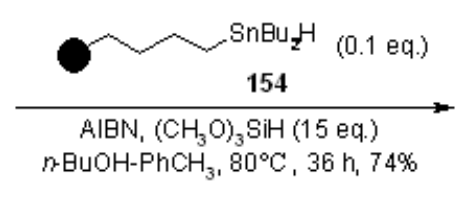

2 more derivatives: yields: $65-70 \%$

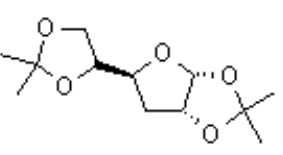

156

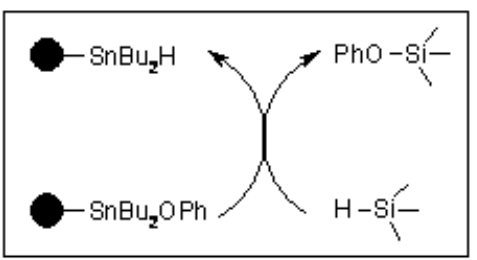

An alternative was chosen and found in trimethoxysilane, giving good results. The reactivation of the tin by-product is highlighted in the scheme (Scheme 49). An alternative to organostannyl hydrides in radical chemistry is represented by the combination organodistannanes/sensitizer/H-donor via, for example $h v$ initiation. Neumann also studied anchored distannanes on SP [63,64], which can easily be prepared starting from the commercial polystyrene. The tin chloride derivative 157 was synthesized by conventional chemistry, and subsequently treated with Bogdanivic's magnesium (Mg-anthracene3THF) to form the distannane 158.

Scheme 50. Neumann's organodistannane.

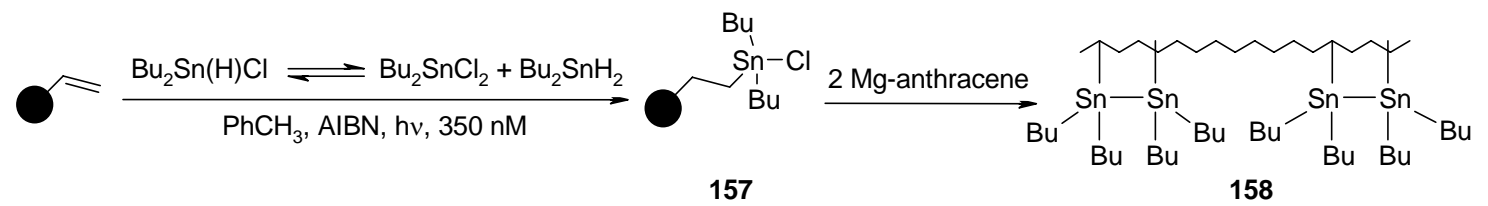


This could be used as the equivalent non-supported reagent in the addition of tert-butyl iodide to acetylenes, yielding similar results. However, the advantage is that after the reaction the polymer containing SnI groups can be recovered by filtration, and completely regenerated in a straightforward manner by reaction with Bogdanivic's magnesium.

Scheme 51. Addition of tert-butyl iodide to acetylenes.

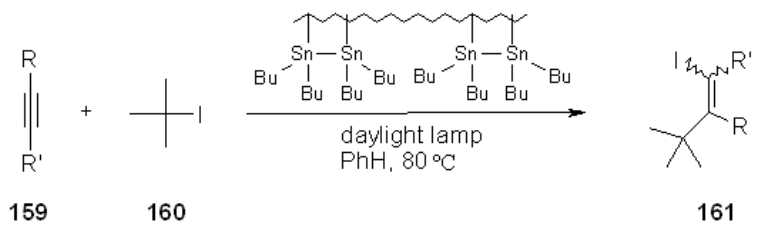

Interestingly, when the polymer was treated with the bromide $\mathbf{1 6 2}$ in the presence of acetone as long-lived triplet sensitizer, and 2-propanol as weak $H$-donor, the corresponding radical 163 thus generated evolved in a somewhat unexpected manner. In fact, instead of the anticipated reduction, a cyclization product was obtained.

Scheme 52. Resin 158-mediated radical cyclization.

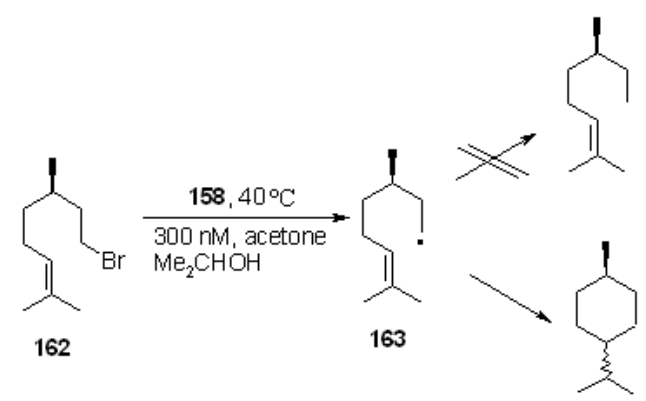

Generally speaking, the possibility to chose the $H$-donor strength (and, therefore, to direct the radical reaction in one of the competitive pathways), the ease of separation of the tin-residues and the little contamination, make this option reagent a feasible suitable choice.

Enholm and colleagues [65] studied the use of allystannane anchored to SP. This toxic reagent is used in the allylation of organohalides (or other homolytic functions) under radical conditions and suffers of the common problems encountered with tin-containing reagents. The use of supported tin reagent leads to ease of purification and minimal contamination of the products. In order to enhance the rates of the intermolecular reaction at test, it was chosen a soluble non-cross-linked polystyrene support (Scheme 53), which induces a 100-fold increase in the rate of intermolecular radical reaction compared to the heterogeneous classical SP reactions.

Scheme 53. Enholm's allylstannane.

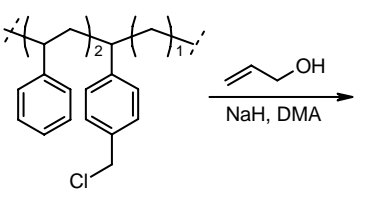

164

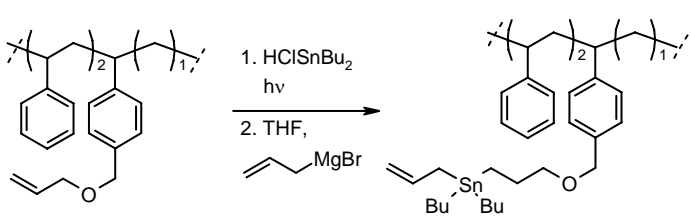

165 
It was found that the best combination < number of reactive sites-polymer properties> led to a $33 \%$ of loading capacity. Test reaction is shown in Scheme 54.

Scheme 54. Application of allylstannane 166.

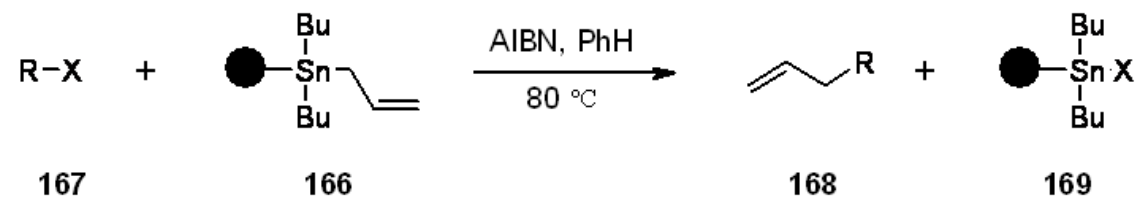

Without going through the details, it is worth underlining some features of the methodology: electrondeficient substrates worked best, while electron-rich did not work at all. This behavior was attributed to the "philicity" of the generated tin radical, which is rather electron-rich (nucleophilic), generating a mismatch with the radical substrate. Interestingly, tertiary radicals, that gave no reaction in solution, gave a 50\% yield. Finally, this procedure displayed a strong regioselectivity towards dihalides substrates, yielding a product of single allylation, strongly preferring electron-deficient radicals. In an extension of the precedent methodology, the same group [66] performed the preparation of another organotinsupported reagent for the reduction of organohalides (Scheme 55).

Scheme 55. Enholm's reduction of organohalides.

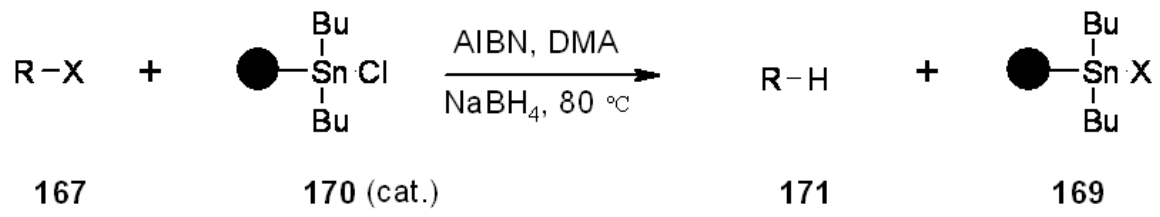

The preparation of $\mathbf{1 7 0}$ was performed following the synthesis depicted in Scheme 53, where $\mathbf{1 6 5}$ was only treated with $\mathrm{HClSnBu}_{2}$. The catalytic methodology used by Enholm is well established [67]. It is widely used for radical reduction of organohalides and represents a useful way to avoid the excess of tin-derivatives, toxic and difficult to separate. The application of the catalytic strategy to the SP allows a low loading of tin-supported reagent and, in addition, the use of a soluble support speeds up the reaction times compared to heterogeneous analogue reactions. Primary alkyl halides reacted quickly with 0.1 eq. of $\mathbf{1 7 0}$ and when the amount of supported reagent was lowered, some reactions took longer. It is worth noting that use of a dihalides with only 1 equivalent of $\mathbf{1 7 0}$ gave only one product of reduction.

Contamination with tin was evaluated via degradation of the products followed by inductively coupled plasma mass spectrometry (ICP-MS), showing the presence of very low residual amount of the metal (around $10 \mathrm{ppm}$ ).

In a very early example of solid-supported organotin reagents, Ueno and coworkers [68] compared insolution radical cyclizations under classical conditions $\left(\mathrm{Bu}_{3} \mathrm{SnH} / \mathrm{AIBN}\right)$ with the use of polymer-bound radical carrier $\left(\mathrm{Bu}_{3} \mathrm{SnCl} / \mathrm{NaBH}_{4}\right)$ for the synthesis of 2-alkoxytetrahydrofurans (Scheme 56). 
Scheme 56. Ueno's synthesis of 2-alkoxytetrahydrofurans.

1)<smiles>[R19]C=CCOC(O[R18])C([R1])Br</smiles>

173

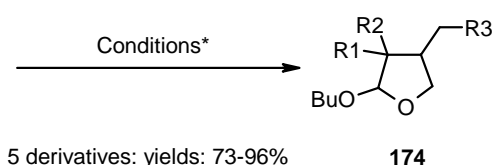

5 derivatives: yields: $73-96 \%$

$\mathrm{R}^{1}, \mathrm{R}^{2}=\mathrm{H},-\left(\mathrm{CH}_{2}\right)_{5} ; \mathrm{R}^{3}=\mathrm{H}, \mathrm{Me}, n-\mathrm{Pr}$

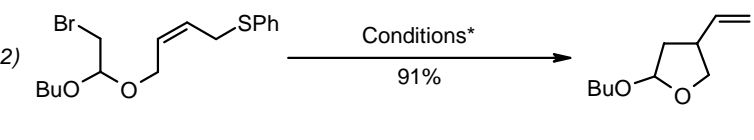

3)<smiles>C=CCOC1OCCCC1Br</smiles>

177

178

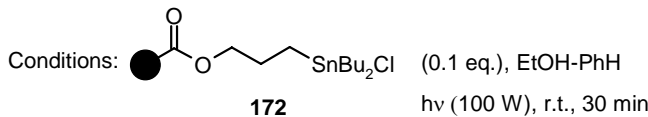

It was found that the reactions were higher-yielding when polystyrene-bound organotin chloride $\mathbf{1 7 2}$ was used as radical carrier. The supported reagent could be used several times after appropriate washing.

Trialkylgermanium hydrides have shown a high potential in radical chemistry often hindered by their high costs, due to their lower toxicity in comparison to that of the corresponding organostannane.

In the need of new reagents, Mochida's group [69] studied the synthesis of polymer-supported germanium hydrides, either via functionalization of polystyrene or by addition to the polymerization mixture of a suitable germanium-derivatized styrene. The supported reagents were tested for the reduction of organic halides, finding comparable results to known in-solution analogous reactions. In addition, it was demonstrated the opportunity of regeneration of the insoluble reagent by reduction with $\mathrm{LiAlH}_{4}$.

Bowman's group [70] also performed the synthesis of resin-bound germanium hydrides. Examples of their applications, which always gave reasonable results, are shown below (Scheme 57).

Scheme 57. Bowman's use of SP-germanium hydrides.

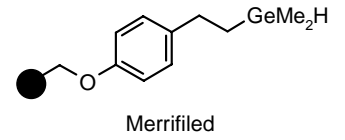

179

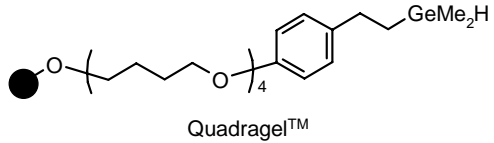

180

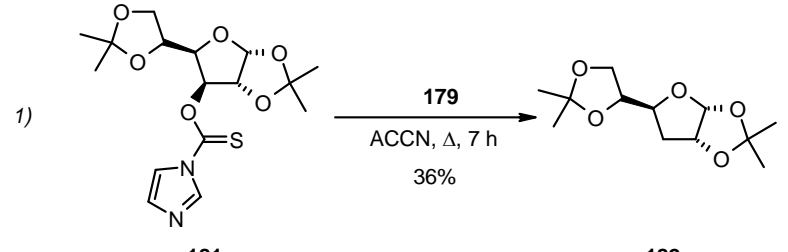

2)
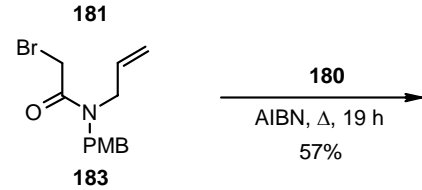<smiles>CC1CC(=O)N([18OH])C1</smiles>

184<smiles>C=CCOc1ccccc1I</smiles>

185

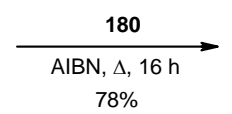

$78 \%$

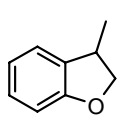

186

In the quest of radical sources on SP, the work presented by Murphy's group [71] represents an alternative to the immobilized organostannanes previously described. Tetrathiofulvalene (TTF) is the promoter for the radical-polar crossover reaction [72-74], a methodology used to generate aryl radicals from aryldiazonium 
salts. The reaction run in solution suffers from the need for separation of TTF by-products and Murphy's goal was immobilization of the promoter in order to simplify work-up (Scheme 58).

Scheme 58. Murphy's synthesis of PS-TTF.

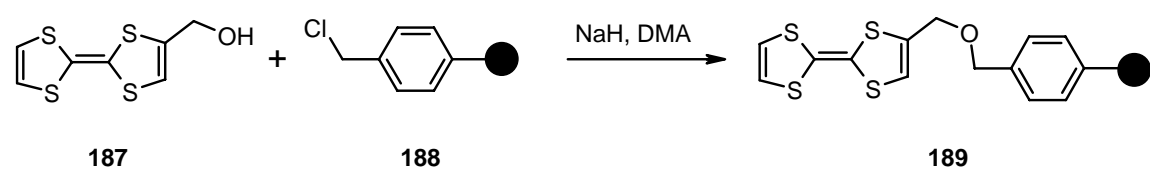

Hydroxymethyltetrathiofulvalene 187 was prepared and loaded to a gel-type resin and used for test in radical cyclization reactions, as the example shown below (Scheme 59).

Scheme 59. Murphy's use of PS-TTF.

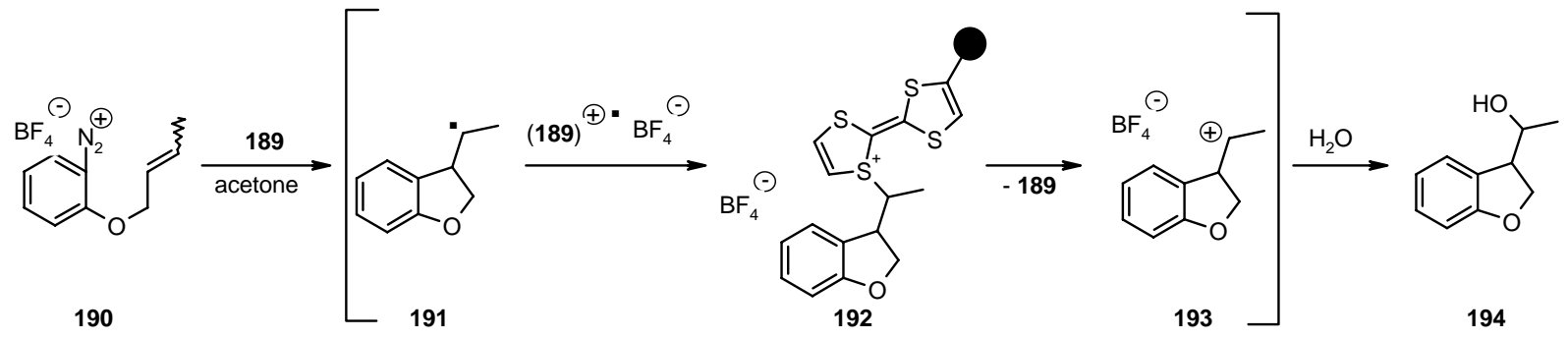

The reactions tested were successful, with comparable results with the homologous run in solution, even if slightly lower-yielding. A major concern was the presence of side-products such as the deoxygenated analogue of 194, possibly arising from $\mathrm{H}$-abstraction by the radical intermediate 191 from the polymer benzylic hydrogens, or products of reaction of cation 193 with the aryl backbone of the resin. In both cases, it was proved that none of these concerns was factual and the reactions resulted rather clean. In addition, contamination of the products from TTF by-products was absent and it was also proven that the SP-TTF could be recycled after regeneration by reduction with $\mathrm{NaBH}_{4}$ in methanol.

Giacomelli and colleagues [75] successfully attempted a useful extrapolation of the classic Barton-Crich thiohydroxamates chemistry [76] in a work where a new polymer-supported radical source was prepared and tested. $N$-hydroxythiazole 2(3)-thione 195 was synthesized and loaded on a Gly-Wang resin to obtain radical source 196 (Scheme 60).

Scheme 60. Giacomelli's interpretation of Barton-Crich thiohydroxamates chemistry.

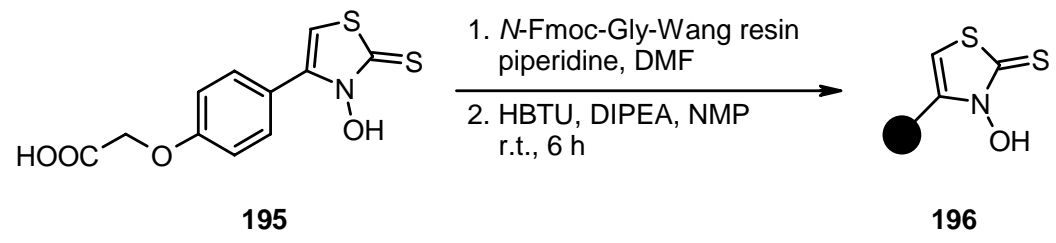

The polymer-supported reagent was then tested for the generation of alkyl radicals (Scheme 61), where $\mathrm{R}=\mathrm{C}\left(\mathrm{CH}_{3}\right)_{3} ; \mathrm{CH}_{2} \mathrm{Ph} ; \mathrm{CH}(\mathrm{Me}) \mathrm{CH}_{2} \mathrm{Ph} ; \mathrm{CH}_{2} \mathrm{CH}_{2} \mathrm{CH}(\mathrm{NHBoc}) \mathrm{COO} t-\mathrm{Bu}$ ) and alkoxyl radical (Scheme 62). 
Scheme 61. Use of resin 196 for the generation of alkyl radicals.

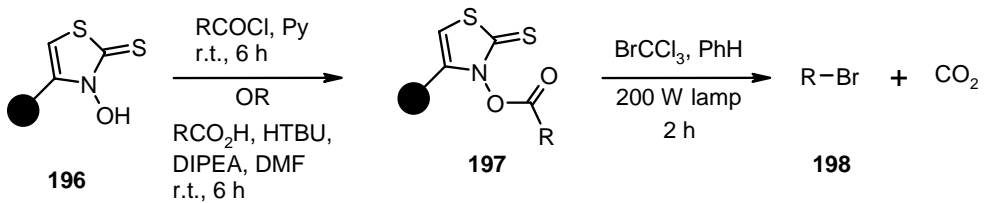

Scheme 62. Use of resin 196 for the generation of alkoxyl radicals.

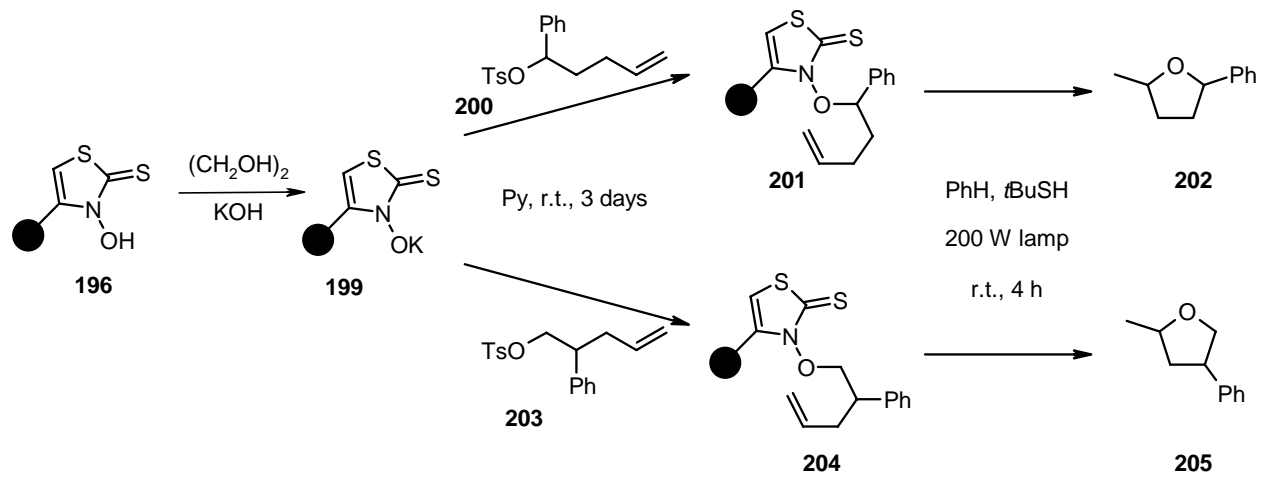

In both cases the reactions were successful, proving this methodology valid for the generation of radicals and having the additional advantage of ease of purification compared to the often tedious experience of purifying the in-solution analogues.

Transition-metal-catalyzed atom transfer radical cyclization (ATRC) represents an alternative, tin-free, non-reductive, catalytic, radical cyclization methodology. Clark's group [77,78] studied the efficiency of this method for the construction of small molecule by anchoring the ligand to SP. In a first attempt [77], functionalized silica was used for the support of the $\mathrm{N}$-alkyl-2-pyridylmethanimine ligands (Scheme 63).

Scheme 63. Clark's ATRC approach.
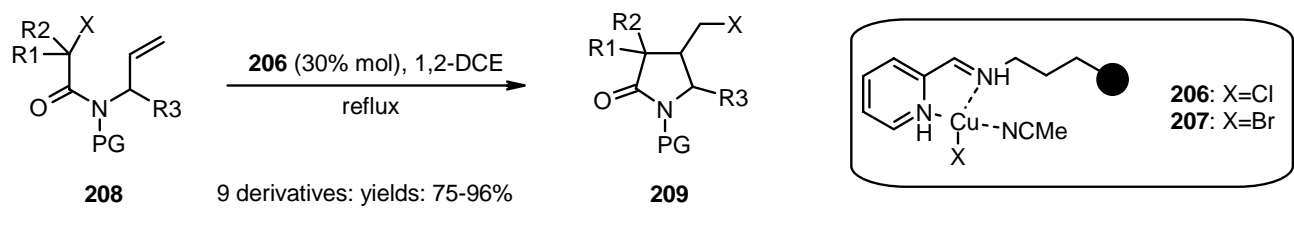

$\mathrm{R}^{1}$ or $\mathrm{R}^{2}=\mathrm{H}, \mathrm{Cl}, \mathrm{Me}, \mathrm{Ph} ; \mathrm{R}^{3}=\mathrm{H}$ or $\mathrm{Me} ; \mathrm{X}=\mathrm{Cl}$ or $\mathrm{Br} ; \mathrm{PG}=\mathrm{Ts}, \mathrm{Bn}, \mathrm{H}$.

All the reactions were high-yielding, the major difference among them being the time necessary to get to completion (from 1 to $36 \mathrm{~h}$ ). The stereochemistry is not shown, although the trans isomer resulted to generally be the major one. The catalysts 206 and 207 were prepared by treatment of the immobilized ligand with $\mathrm{CuX}$ and 206 was also reused at least 3 times, giving still good yields $(92 \rightarrow 90 \rightarrow 86 \%$ ), but longer reaction times $\left(3 \rightarrow 24 \rightarrow 36 \mathrm{~h}\right.$ ), a deactivation probably caused by the formation of an inactive $\mathrm{CuCl}_{2}$ complex. In a successive paper, Clark [78] used different supports and several ligands, in order to determine the best combination to improve the efficiency of the reaction (Scheme 64). 
Scheme 64. Clark's catalyst improvements.

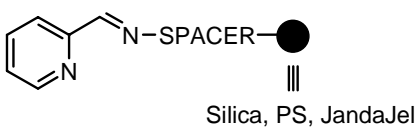

SPACER $=-\left(\mathrm{CH}_{2}\right)_{3},-\mathrm{CH}_{2}-$

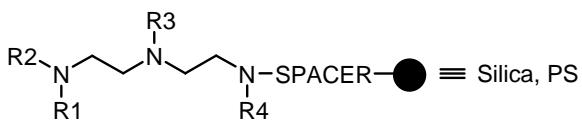

$\mathrm{R}^{1}=\mathrm{R}^{2}=\mathrm{Me}, \mathrm{Et} ; \mathrm{R}^{3}=\mathrm{Me}, \mathrm{Me}_{2} \mathrm{NCH}_{2} \mathrm{CH}_{2^{-}}, \mathrm{R}^{4}=\mathrm{Me}$ SPACER $=-\left(\mathrm{CH}_{2}\right)_{3},-\mathrm{CH}_{2}-$

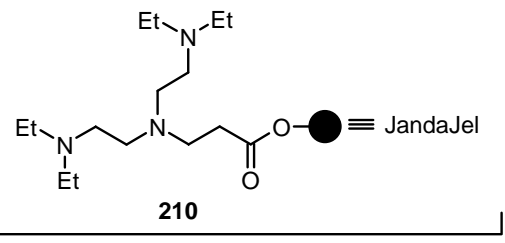

II

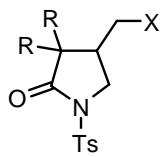

212

Running the above reaction at room temperature, some differences among the catalysts (obtained after treatment of supported ligands of type I or II with $\mathrm{CuX}$ ) arose, since there was a clear superiority of those of type I vs. type II, in terms of reaction times and conversions (yields were, however, rather high). Raising the temperature to reflux, the reaction times were less than $30 \mathrm{~min}$, independently of the catalyst used. Some more substrates were investigated, giving interesting results. For instance, in (Scheme 65) the catalyzed radical addition to a terminal alkyne is described. The absolute yields were high (88-98\%), independently of the ligand used, even if type I catalysts were slightly better. $E: Z$ ratio was almost the same for the ligand screening, however, the presence of reduced product 216 varied between 11-6\%, being 0\% when silica type I was used.

Scheme 65. Example of radical addition to a terminal alkyne.

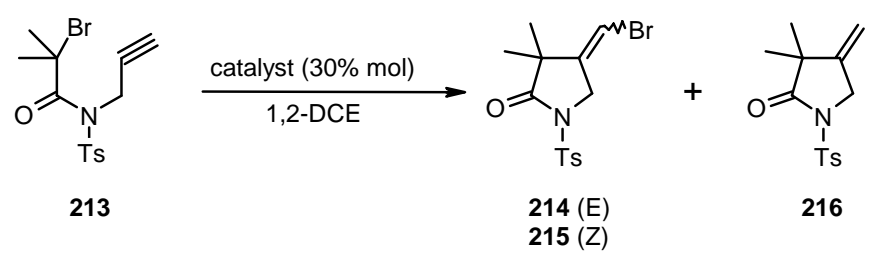

In Scheme 66 is depicted a 5-endo-trig radical polar crossover addition, a normally disfavored process following Baldwin's rules [79,80], but feasible when polyamine-copper catalysts such as type II (Scheme 64) are used [81]. In this case a mixture of 218 and 219 was obtained. The best yield (100\%) was found when $\mathbf{2 1 0}$ was used as ligand, while 220 was isolated in low yield (15\%) as the sole product when type I Janda Jel ligand was used.

Scheme 66. Clark's 5-endo-trig radical polar crossover addition.

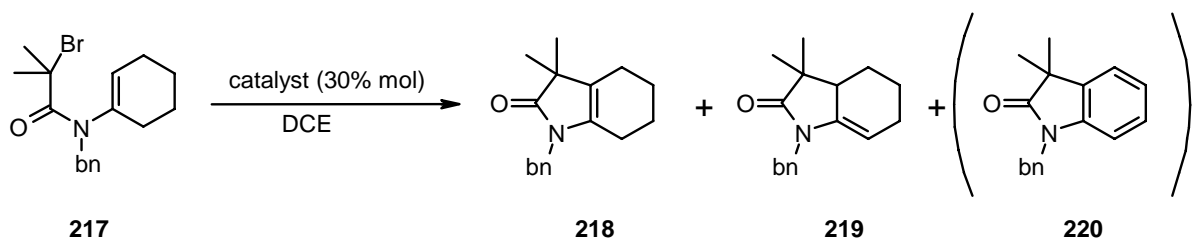

Finally, in Scheme 67 a 4-exo-trig cyclization is represented. This also is a disfavored process according to Baldwin's rules, which was successful only when $\mathbf{2 1 0}$ was used as ligand. 
Scheme 67. Clark’s 4-exo-trig radical cyclization.<smiles>CC(Br)(Br)C(=O)N(C=C1CCCCC1)c1ccccc1</smiles>

221

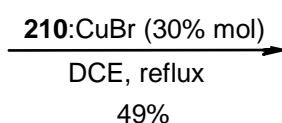

$49 \%$

222

Nagashima and coworkers [82] studied the same reaction, using different copper complex as the catalyst. In this case, in fact, the metal-ligand complex used for the catalysis was immobilized on a very peculiar SP the (PMHS). The siloxane gel [bipy]@Si contained, embedded, a bypyridine ligand and it was synthesized from PHMS and 4,4'-bis(allyloxymethyl)-2,2'-bipyridine (DAbipy) by the platinum-catalyzed hydrosilylation of alkenes. The catalyst was then tested on several radical precursors (Scheme 68).

Scheme 68. Nagashima's Cu-catalytic complex.

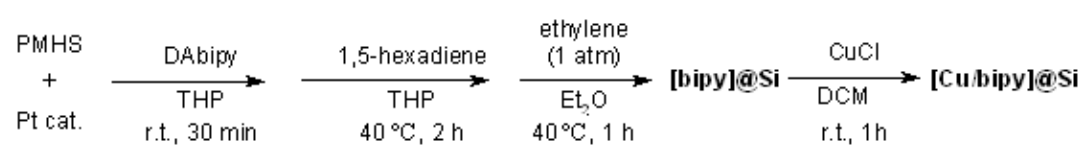

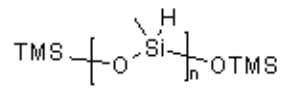

PMHS

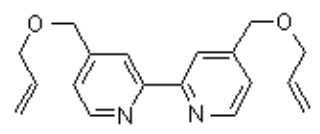

DAbipy

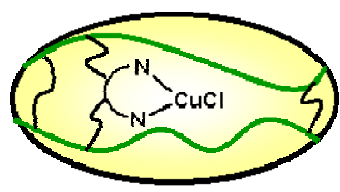

[Cubipy]QSi

The synthesis of the actual catalyst [Cu/bipy]@Si was completed after treatment of [bipy]@Si with $\mathrm{CuCl}$. The metal was easily soaked into the gel, forming the active complex, which resulted more stable to air than the not-embedded version and that can be filtrated from the organic solvents used for the reaction and re-used with small loss of TON. Metal leakage inferior to the detection limit was observed.

Scheme 69. Application of [Cu/bipy]@Si to 5-exo-trig radical cyclization.

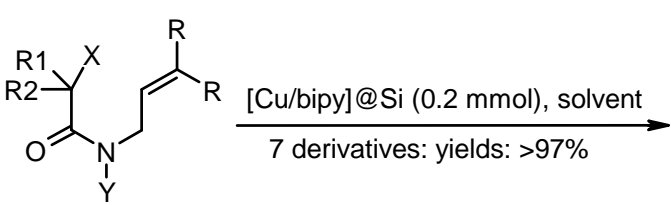

223

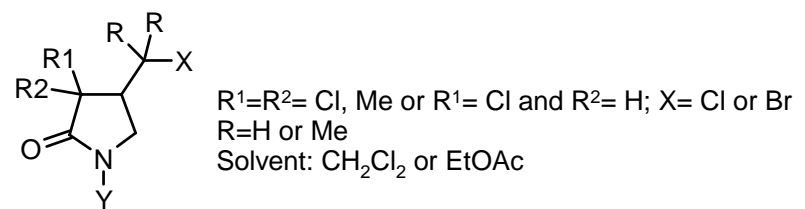

224

Scheme 70. Application of [Cu/bipy]@Si to 4-exo-trig radical cyclization.<smiles>CC(C)=CN(Cc1ccccc1)C(=O)Cl</smiles>

225

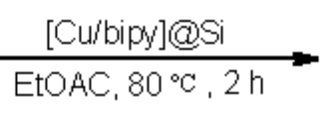

$99 \%$<smiles>CC(C)(Cl)C1N(Cc2ccccc2)C(=O)C1(Cl)Cl</smiles>

226 
The reactions shown in Schemes 69 and 70 were all pretty successful (97-99\%) and when the reaction gave low yields at room temperature, simple heating gave almost complete conversions.

Hypervalent iodine reagents can be used for the generation of alkoxy radicals under irradiation conditions. Teduka and Togo [83] used polystyrene-supported (diacetoxyiodo)benzene for the synthesis of 2-substituted 1,3-dioxolanes (Scheme 71), 2-substituted tetrahydrofurans (Scheme 72) and 2-substituted 1,3-dioxanes (Scheme 73).

Scheme 71. Togo's synthesis of 1,3-dioxolanes.

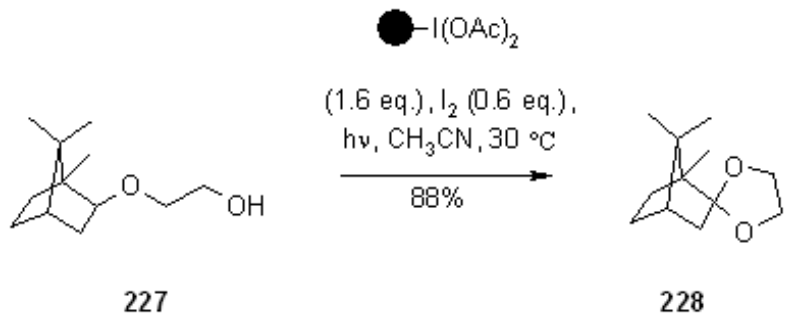

Scheme 72. Togo's synthesis of 2-substituted tetrahydrofurans and dioxolanes.

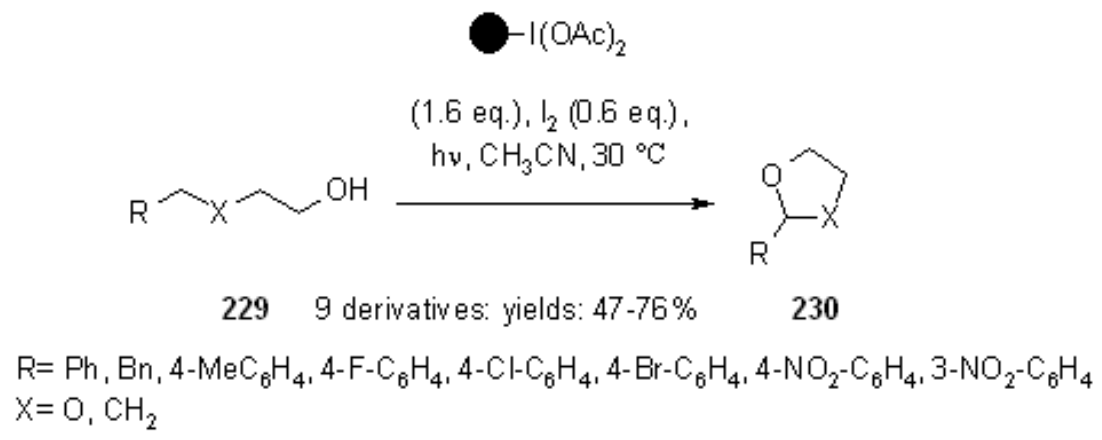

Scheme 73. Togo's synthesis of 2-substituted-1,3-dioxanes.

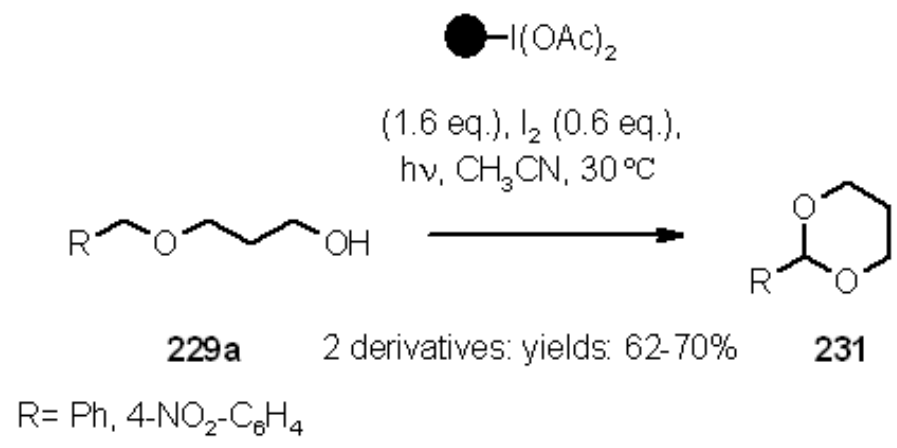

Oxidation of alcohols to the corresponding aldehyde or ketone represents a very common reaction in daily organic synthesis; however, there is always a need for new and milder methods for sensitive molecules, especially in SP organic chemistry. Kashiwagi's group [84] studied a mild and efficient methodology, using a catalytic two-phase system, under basic conditions. (Scheme 74) 
Scheme 74. Kashiwagi’s catalytic two-phase system.
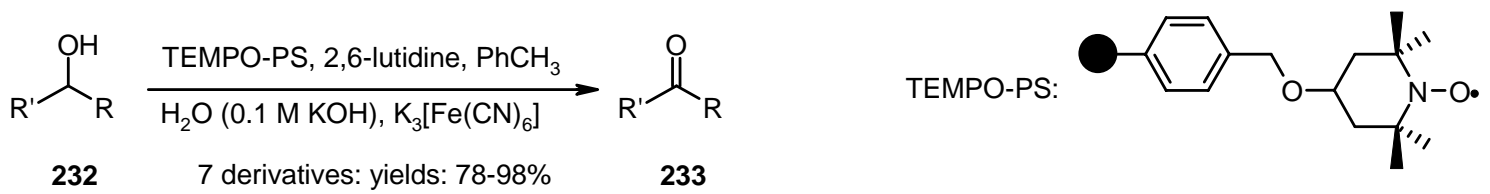

Proposed mechanism:

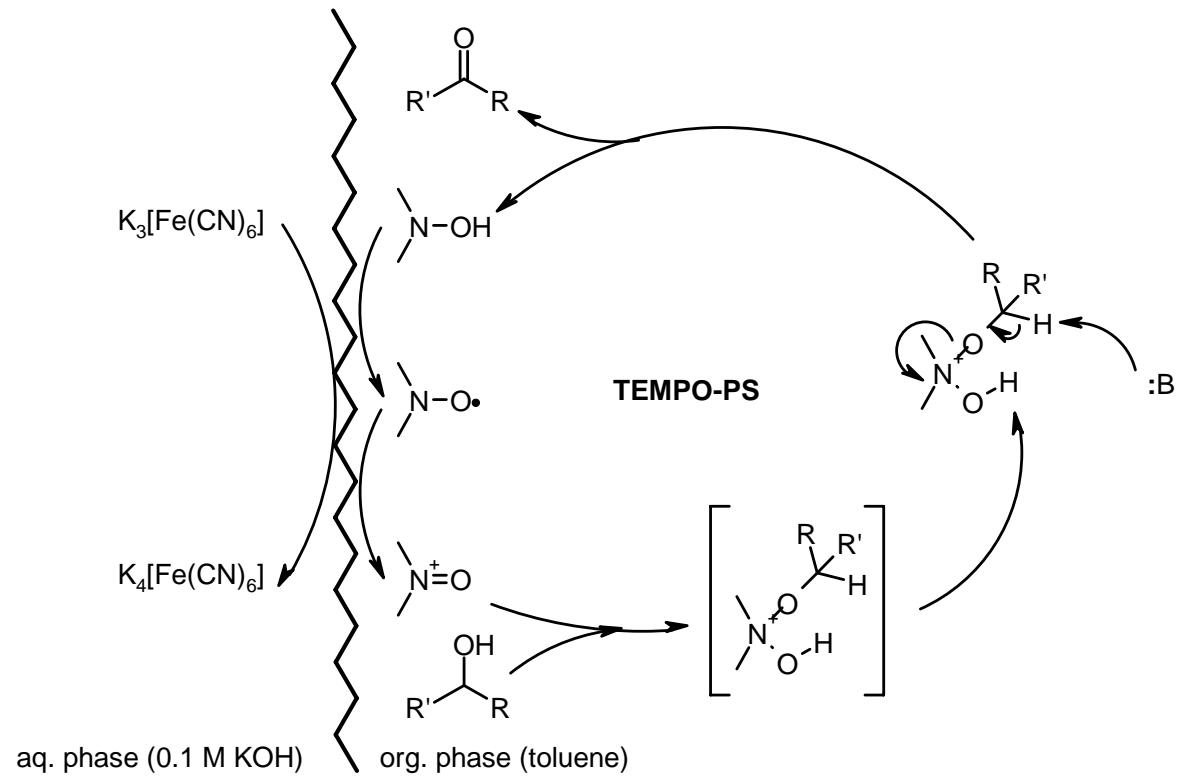

The oxidation mechanism is displayed above and consists of an interface interaction between the oxidant $\left(\mathrm{K}_{3}\left[\mathrm{Fe}(\mathrm{CN})_{6}\right]\right)$ in aqueous, basic solution and the hydroxylamine polymer-bound (TEMPO-PS) in organic phase (toluene), formed after oxidation of the alcohol 232. The base (2,6-lutidine) was necessary to close the catalytic cycle, yielding the desired carbonyl 233. Interestingly, in was found that this method has a rather high chemoselectivity, preferring primary alcohols oxidation to secondary alcohols.

\section{Exploitation of Radical Chemistry in SP Synthesis}

The importance of radicals in organic chemistry has grown considerably over the past three decades and several methodologies have been studied and set up to face the need of new routes for the synthesis of target molecules or classes of compounds. In this section, the studies and applications of those methodologies to SP will be discussed. Following our goal to provide a reference for the day-today use of solid-phase radical reactions, we have not based our classification on mechanistic grounds, but more simply on the formal way to generate a new bond.

\subsection{Carbon-Carbon Bond Formation through Intermolecular Reactions}

\subsubsection{Substitutions Alpha to Carbonyl Groups}

The first examples of intermolecular free radical allylation reaction on solid support was reported by Sibi and Chandramouli [85], for the synthesis of simple $\alpha$-allyl acetic acids (Scheme 75). 
Scheme 75. Sibi’s allylation reaction on supported $\alpha$-bromo.<smiles>[R]C([R])(Br)C(=O)OCC</smiles>

234

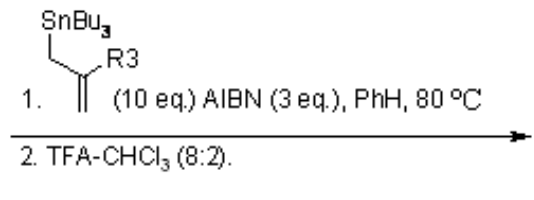

10 derwatives yields: $58-95 \%$<smiles>[R3]C(=C)CC([R1])([R])C(=O)O</smiles>

235

$\mathrm{R}^{\mathrm{l}}=\mathrm{H}_{1} \mathrm{CH}_{3} ; \mathrm{R}^{2}=\mathrm{CH}_{3}, \mathrm{Ph}, n-\mathrm{C}_{4} \mathrm{H}_{9,} \mathrm{CH}\left(\mathrm{CH}_{3}\right)_{2} ; \mathrm{R}^{3}=\mathrm{H}_{1} \mathrm{CH}_{3}, \mathrm{OAC}_{1} \mathrm{CO}_{2} \mathrm{CH}_{3}$.

Wang resin was used as the solid support and the reactions were carried out using allyltin derivatives. As shown in the scheme, the reactions were rather successful and allylation was more effective (95\% yield) when $\mathrm{R}^{3}$ was either and electron-donating (OAc) or electron-withdrawing $\left(\mathrm{CO}_{2} \mathrm{CH}_{3}\right)$ group, while substitution around the $\mathrm{C}$-centered radical gave lower yield $(58 \%)$ for $\mathrm{R}^{1}=\mathrm{R}^{2}=\mathrm{CH}_{3}$. Allyl transfer reaction was studied also by Enholm's group [86] who worked on a soluble support (Scheme 76).

Scheme 76. Enholm's allyl transfer reaction.
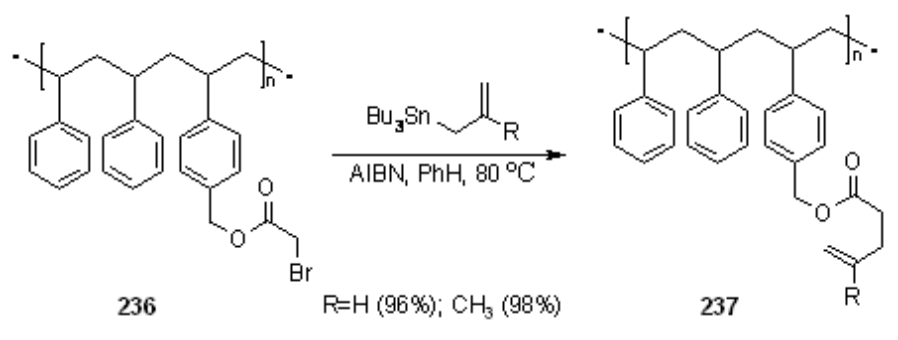

The polymer (non-cross-linked polystyrene) was functionalized to obtain the radical precursors. As shown in Scheme 76, 236 could be reacted with allyltributyltin derivatives under radical conditions, allowing allylation of the carbonyl $\alpha$-carbon.

Scheme 77. Enholm's allyl transfer reaction.
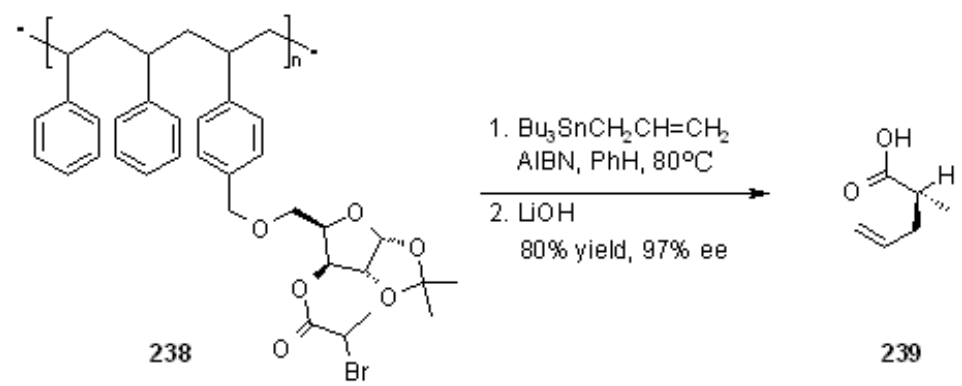

Allylation of the same radical precursor ( $\alpha$-bromo carboxylate) linked this time to the polymer by means of a sugar moiety is shown in Scheme 77. When the reaction was performed in solution, use of a Lewis acid increased stereoselectivity. However, in the case shown in Scheme 77 any Lewis acid (LA) used gave premature cleavage. Fortunately, in this case a good stereoselectivity (97\% ee) was found without any LA addition: this can be explained invoking the Lewis acidity of $\mathrm{Bu}_{3} \mathrm{SnBr}$ (formed during the reaction), which coordinates preferentially to one face of the sugar molecule directing the 
allyl attack. Enholm studied a series of intermolecular radical reactions also exploiting ROMP polymers [87] (see below).

Scheme 78. Enholm's use of ROMP polymers for the synthesis of 241.

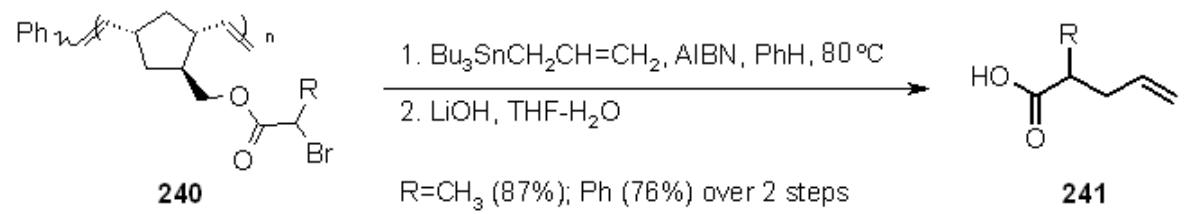

Scheme 79. Enholm's use of ROMP polymers for the synthesis of 243.

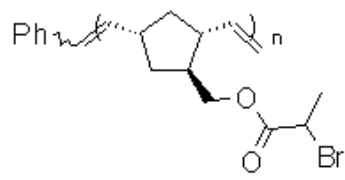

242

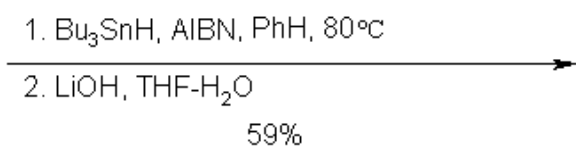

$59 \%$<smiles>[R]C(=O)O</smiles>

243

Scheme 80. Enholm's use of ROMP polymers for the synthesis of $\mathbf{2 4 5}$.<smiles>[B]C(P)C(=O)OC[C@@H]1[C@@H](/C=C/c2ccccc2)C[C@H](C#N)[C@H]1COC(=O)C(Br)c1ccccc1</smiles>

244

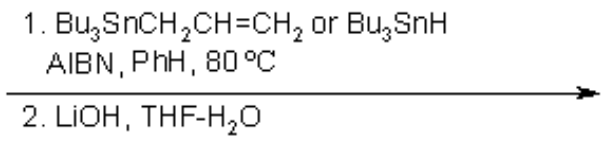

$\mathrm{R}=$ ally $(78 \%) ; \mathrm{H}(67 \%)$ over 2 steps<smiles>[R]C(C(=O)O)c1ccccc1</smiles>

245

The polymers 240, 242 and 244 (Schemes 78-80) were prepared by building the appropriate norbornene precursors that were subsequently treated with Grubbs' catalyst (see below). Treatment of the so obtained soluble polymers under radical conditions gave the desired products of allylation (Scheme 78) or reduction (Scheme 79) in good yields. A tremendous advantage of this type of polymers is represented by the presence of at least one molecule of substrate in each monomer, giving a $100 \%$ loading capacity to the polymer. In the case of Scheme 80 , where each monomer contains two reactive sites, a $200 \%$ theoretical loading of the resin was obtained.

Linhardt and coworkers [88] used $\mathrm{SmI}_{2}$ as a SET reagent for the $C$-glycosilation of Neu5Ac on SP. Since carboxyl TentaGel resin failed to give any desired product, the reaction was run on a less hydrophilic material, namely a functionalized controlled pore glass (CPG). The glass was derivatized in order to present an amine group handle (Scheme 81). Methyl ester of neuraminic acid was first esterified with a spacer that was subsequently attached to the support. As shown in the scheme below, the results of the $C$-glycosilation reaction were rather satisfactory. 
Scheme 81. Linhardt's $C$-glycosilation.

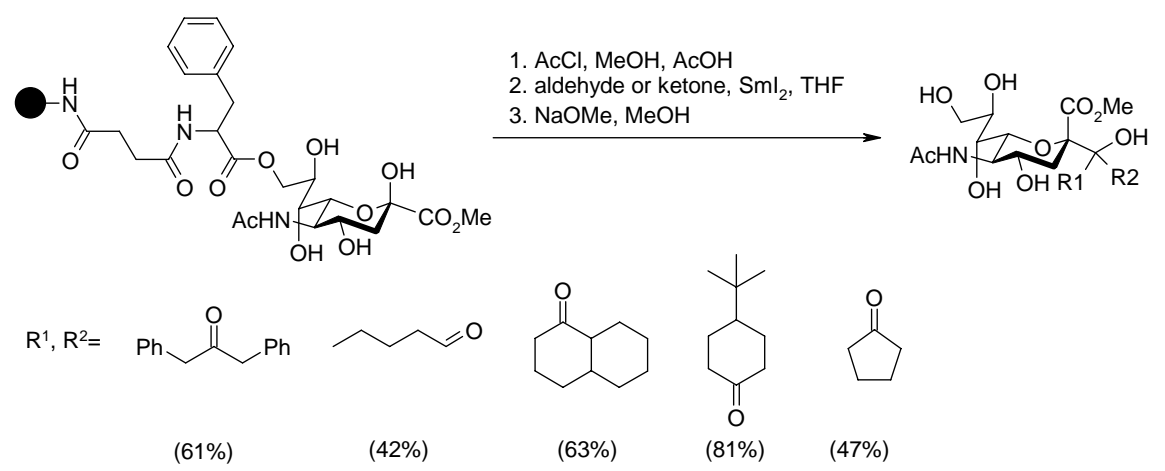

\subsubsection{Radical Addition to Alkenes}

Radical addition to unsaturated $C$ - $C$ bonds represents one of the most common reactions in radical chemistry. The efficacy of the intermolecular versions of such reactions is heavily dependent on the nature of reaction itself. An early example of this chemistry on SP was presented by Zhu and Ganesan [89], that proved the efficiency of alkyl radical addition to acrylates attached to a chosen resin (Scheme 82).

Scheme 82. Ganesan's radical addition to supported alkenes.
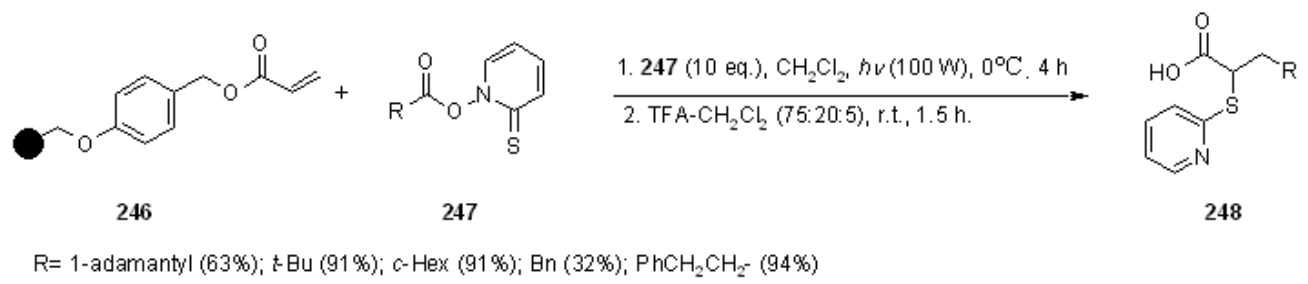

248

$\mathrm{R}=1$-adamantyl $(63 \%) ; \mathrm{t}-\mathrm{Bu}(91 \%) ; \mathrm{c}-\mathrm{Hex}(91 \%) ; \mathrm{Bn}(32 \%) ; \mathrm{PhCH}_{2} \mathrm{CH}_{2}-(94 \%)$

Scheme 83. Ganesan's radical addition using Rink resin.

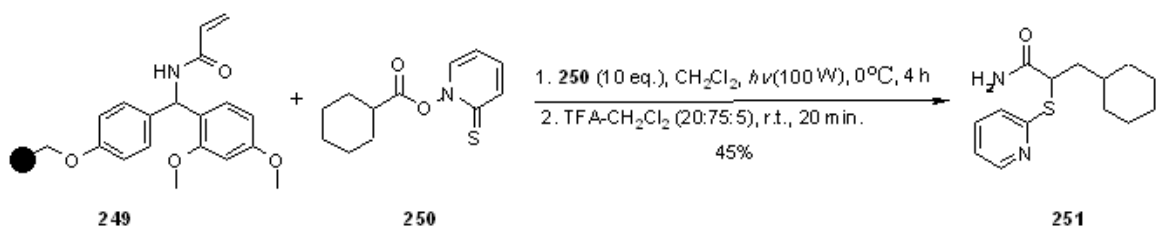

The alkyl radicals were generated by photolysis of the corresponding Barton esters $\mathbf{2 4 7}$ and addition was generally good, with the exception of the stabilized benzyl radical that gave poor conversion (yields refer to the use of PS-Wang resin, as Tentagel-Wang gave lower yields). The reaction was performed using a Wang (Scheme 82) or a Rink (Scheme 83) resin. In the latter case yield was lower, a result in accord to the in-solution reaction, probably due to the differences between acrylate ester 246 and acryl amide 249.

1,2-Radical initiated addition of haloalkanes to $C=C$ bonds is employed in commercial routes for the preparation of dihaloethenylcyclopropane carboxylic acids, and the development of cleaner and easier methods has brought Kumar and colleagues [90] to attempt the reaction anchoring the olefin to SP (Scheme 84). 
Scheme 84. Kumar's radical addition to supported alkenes.

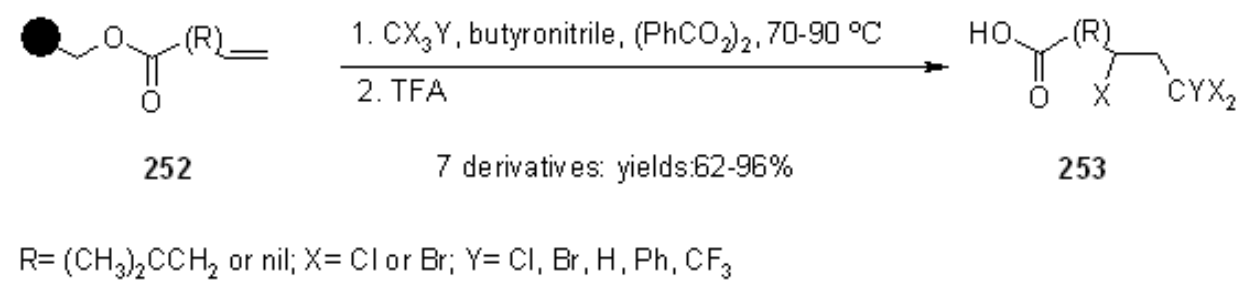

The olefins were linked to Wang or Merrifiled resins by their carboxylic unit and the reactions were run in the presence of butyronitrile as a cosolvent (except when $\mathrm{CCl}_{4}$ was used as reagent). Yields varied from moderate to high, for reaction times between 24 and $48 \mathrm{~h}$, making this method effective, but rather slow. The synthesis of racemic $\alpha$-amino acids was described by Yim [91] that applied the mercury method for the generation of alkyl radicals in the presence of polymer-supported dehydroalanine (Scheme 85).

Scheme 85. Yim's radical addition to supported alkene.<smiles>C=C(NC(C)=O)C(=O)OC</smiles>

254

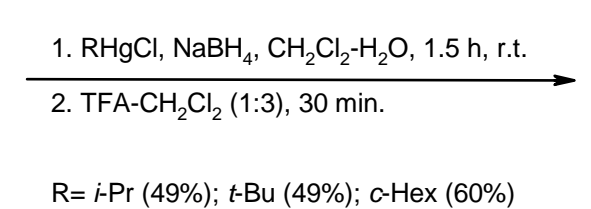

$\mathrm{R}=i-\operatorname{Pr}(49 \%) ; t-\mathrm{Bu}(49 \%) ; c-\mathrm{Hex}(60 \%)$<smiles>[R]C[C@H](NC(C)=O)C(=O)O</smiles>

255

Addition of the alkyl radical on the double bond was effective and after cleavage reasonable yields of the desired products were isolated. When the same reaction was performed under more usual radical conditions $\left(\mathrm{Bu}_{3} \mathrm{SnH}, \mathrm{AIBN}, t\right.$ - $\mathrm{BuI}, \mathrm{PhH}$, under reflux), only trace amounts of the product $(8 \%)$ were isolated, while prolonged heating led only to decomposition. The reason for this behavior was attributed to the poor swelling of the Wang resin in the solvent used in the attempt (benzene).

Caddick's group [92] prepared a small array of amide derivatives by intermolecular radical addition of C-centered radicals to an activated acrylate anchored to a resin. Aminomethyl-polystyrene resin was chosen as the SP where to anchor the acrylate ester of 2,3,5,6-tetrafluoro-4-hydroxybenzoic acid (256). The choice of such electron-poor acrylate was made in order to facilitate the radical reaction and obtain an amide after cleavage with an appropriate amine. The general synthetic scheme of this highyielding methodology is represented in Scheme 86.

Scheme 86. Caddick's radical addition to supported alkene.

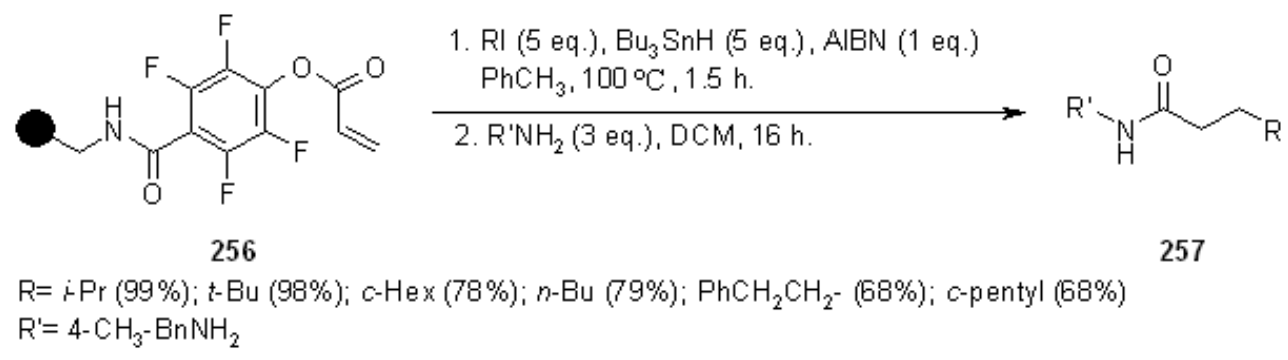


In Schemes 87 and 88 the use of carbohydrate derivatives as radical precursors (258 and 260 respectively) is shown. In these cases, $\alpha$-amino acids were used as amines, although the yields were lower compared to the previous case.

Scheme 87. Synthesis of compound 259.

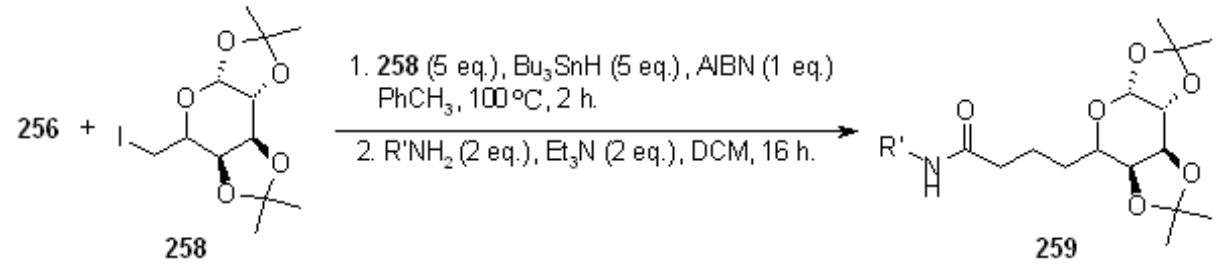

$\mathrm{R}^{\prime}=4-\mathrm{CH}_{3}-\mathrm{BnNH}_{2}(53 \%) ;$ PhOEt (44\%); TrpOMe (57\%); TyrOMe (21\%); SerOMe (16\%)

Scheme 88. Synthesis of compound 261.

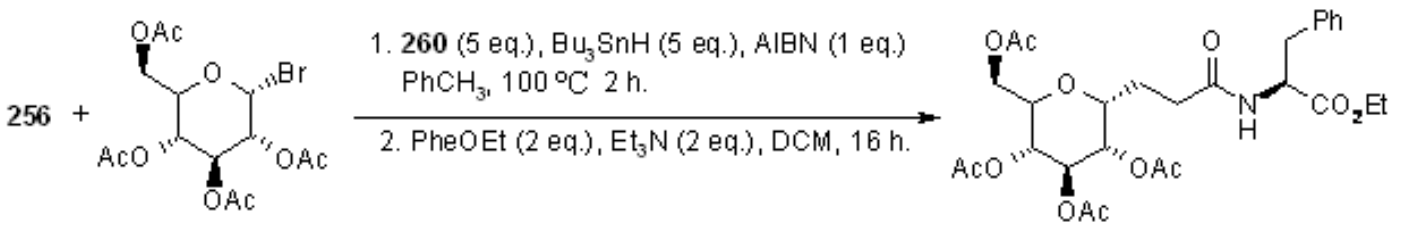

260

261

\subsubsection{Radical Addition to Oxime}

Among the literature references regarding the use of radical chemistry on SP, Naito's group contributed by studying the reactivity of SP-supported oxime ethers. In their works [93-97], they studied the addition of appropriate alkyl iodides to polymer-supported glyoxylic oxime ether, giving the first example of intermolecular radical reaction exploiting triethyl borane or diethyl zinc as radical initiators. The methodology was used for the synthesis of unnatural aminoacids (Scheme 89).

Scheme 89. Naito's radical addition to supported oxime.

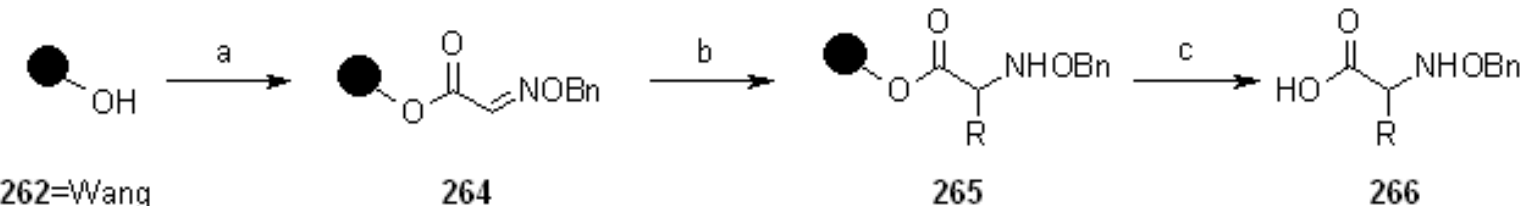

$263=$ TentaGel OH

(a) $\mathrm{HO}_{2} \mathrm{CC}=\mathrm{NOBn}, \mathrm{DCC}, \mathrm{DMAP}, \mathrm{CH}_{2} \mathrm{Cl}_{2}, 20^{\circ} \mathrm{C}, 12 \mathrm{~h}$ (for 262);

$\mathrm{HO}_{2} \mathrm{C}=\mathrm{CNOBn}, 2,6$-dibenzoyl chloride, $\mathrm{Py}, \mathrm{DMF}, 20^{\circ} \mathrm{C}, 12 \mathrm{~h}$ (for 263)

(b) $\mathrm{Et}_{3} \mathrm{~B}, 1$ h or: $\mathrm{Et}_{2} \mathrm{Zn}, 15$ min.; or: $\mathrm{Rl}, \mathrm{Bu}_{3} \mathrm{SnH}_{1} \mathrm{Et}_{3} \mathrm{~B}, 1$ h or: $\mathrm{Rl}_{1} \mathrm{Et}_{3} \mathrm{~B}$ or $\mathrm{Et}_{2} \mathrm{Zn}$;

(c) $\mathrm{TFAlCHCl}_{3}(1: 3, w / \mathrm{w}), 20^{\circ} \mathrm{C}, 30 \mathrm{~min}$.

After loading the oxime to the solid phase (264), a first attempt was made using only $\mathrm{Et}_{3} \mathrm{~B}$ in DCM, which worked as initiator and terminator to afford, after cleavage, aminoacid 266 where $\mathrm{R}=\mathrm{Et}$. Running the reaction with an alkyl iodide $\mathrm{R}-\mathrm{I}$ in the presence of $\mathrm{Bu}_{3} \mathrm{SnH}$, a range of products were obtained, thus, when $\mathrm{R}=i$-Pr, $c$-hexyl, $t$ - $\mathrm{Bu}, s$-Bu, the corresponding unnatural amino acids were 
recovered in good yields, while the unstable primary radical $i$-Bu and the bulky adamantyl gave lower yields. In all the reactions, a small amount $(5-30 \%)$ of $\mathbf{2 6 6}(\mathrm{R}=\mathrm{Et})$ was found as by-product from the competitive addition of ethyl radical arising from $\mathrm{Et}_{3} \mathrm{~B}$. The reaction was also run in the presence of $\mathrm{Et}_{2} \mathrm{Zn}$, which gave comparable results to the $\mathrm{Et}_{3} \mathrm{~B}$; in both cases the radical chain was effective also at low temperatures $\left(-78{ }^{\circ} \mathrm{C}\right)$. Successively, they tried to run the reaction under iodine-atom transfer mechanism, using $\mathrm{Et}_{3} \mathrm{~B}$ and $i$-PrI, but avoiding $\mathrm{Bu}_{3} \mathrm{SnH}$. In this case a large amount of product where $\mathrm{R}=\mathrm{Et}$ was found. The increased competition of the side-reaction was not explained, however, a change in solvent and temperature (toluene, $80^{\circ} \mathrm{C}$ ) as well as the use of a larger amount or alkyl iodide (60 eq.) gave a better selectivity towards the desired product (5.7:1 vs. 2:1). Such selectivity, however, remained lower compared to the reaction run in solution.

In general, when the reaction was run in the presence of an organic solvent, Wang resin proved to be slightly better compared to TentaGel $\mathrm{OH}$. However, when the reaction was tried in aqueous media, which is normally effective in solution, Wang resin did not work at all because of its poor swelling properties. Success was achieved with TentaGel OH resin, where it was necessary to use a solution of $\mathrm{Et}_{3} \mathrm{~B}$ in $\mathrm{THF}$ or $\mathrm{MeOH}$ (compared to the hexane solution used in the previous attempts) to obtain a monophasic solution. The reaction was run in a mixture $\mathrm{H}_{2} \mathrm{O}-\mathrm{MeOH}\left(2: 1\right.$, v/v, at $\left.70{ }^{\circ} \mathrm{C}\right)$, under iodine atom transfer conditions and the overall yields were acceptable though lower compared to the ones run in organic solvents; a comparable amount of ethylated product was obtained.

Although the methodology described by Naito was biased by the presence of the undesired product of ethyl radical addition, the use of aqueous solvents and the absence of the toxic tin hydride make this procedure quite appealing. The same group also studied the stereoselective version [96-99] of the above-described reaction, using a chiral auxiliary (Scheme 90).

Scheme 90. Naito's stereoselective radical addition to supported oxime.

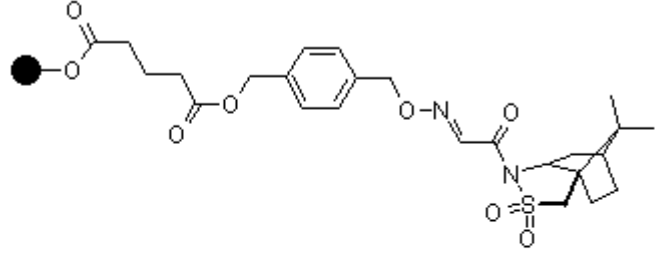

267

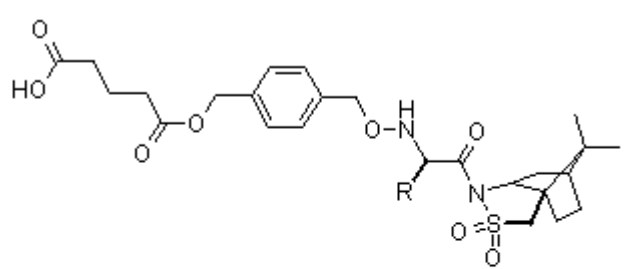

268

(a) $\mathrm{Et}_{3} \mathrm{~B}$ or $\mathrm{Et}_{2} \mathrm{Zn},-78^{\circ} \mathrm{C}$ or: $\mathrm{RI}, \mathrm{Et}_{3} \mathrm{~B}$ or $\mathrm{Et} Z \mathrm{Zn}, \mathrm{RI}-\mathrm{PhCH}_{3}(4: 1$, wi $) 0^{\circ} \mathrm{C}$ (b) $\mathrm{TFAJCH}_{2} \mathrm{Cl}_{2}\left(1: 5\right.$, wiv), $20^{\circ} \mathrm{C}$.

Oppolzer's camphorsultam was chosen as an auxiliary, while the reactions were tested in SP (using Wang resin) and in solution. The oxime was attached to a carboxylic acid derivative which, in the SP version, served as the spacer between the reactive moiety and the polymeric support (compound 267). In a first attempt, it was decided to try ethylation of $\mathbf{2 6 7}$ following the method described in Scheme 88, using $\mathrm{Et}_{3} \mathrm{~B}$ or $\mathrm{Et}_{2} \mathrm{Zn}$ at low temperatures $\left(-78{ }^{\circ} \mathrm{C}\right)$ in order to facilitate stereoselectivity. In both cases the results were very promising, high yields (59-74\%), with little variation between solvents (DCM slightly better than toluene) and radical source $\left(\mathrm{Et}_{3} \mathrm{~B}\right.$ slightly better than $\left.\mathrm{Et}_{2} \mathrm{Zn}\right)$, with diastereoselectivity higher than $95 \%$. In order to prove the general validity of the method, radical addition from a different radical source was tried. At first $\mathrm{Bu}_{3} \mathrm{SnH}$ was used together with an alkyl 
iodide, but results were disappointing: low yield (around 40\%) and a mixture of products were obtained (addition of ethyl radical prevailed). When the reaction was run without the tin hydride, yield improved (78\%), but the great majority was still the undesired product (5:1), which was in contrast with the analogous solution-phase reaction. In order to overcome these problems, the conditions were changed: the reaction was performed using a mixture of R-I (30 eq.) and toluene (4:1) under iodine atom transfer conditions in the presence of $\mathrm{Et}_{3} \mathrm{~B}$ or $\mathrm{Et}_{2} \mathrm{Zn}(5$ eq. $)$, and at higher temperatures $\left(0{ }^{\circ} \mathrm{C}\right)$. This led to higher yields ( $\mathrm{R}=i$-Pr: $69 \% \mathrm{Et}_{3} \mathrm{~B}, 53 \% \mathrm{Et}_{2} \mathrm{Zn} ; \mathrm{R}=c$-hexyl: $58 \% \mathrm{Et}_{3} \mathrm{~B}, 41 \% \mathrm{Et}_{2} \mathrm{Zn}$ ), and higher stereoselectivities ( $>90 \%$ ) compared to the in-solution reactions. The reason for the presence of such large amount of by-product in SP was not explained, however, this tendency increased at lower temperatures, suggesting that triethylborane concentrate on solid-support as Lewis acid, releasing a large amount of ethyl radical around the surface of the polymer.

In an approach similar to the one described by Naito in Scheme 89, Kim and colleagues [100] performed the synthesis of $\alpha$-aminoesters by addition of alkyl radical to an anchored phenylsulfonyl oxime ether (Scheme 91).

Scheme 91. Kim's radical addition to supported oxime ether.

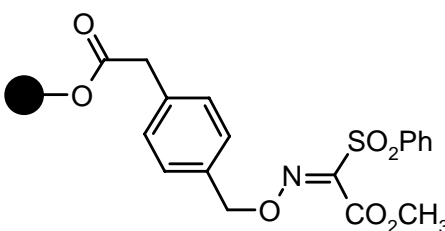

269

$$
\frac{\text { 1. R-I, }\left(\mathrm{Me}_{3} \mathrm{Sn}\right)_{2}, \mathrm{PhH}, h v}{\text { 2. } 1 \mathrm{~N}-\mathrm{HCl} / \mathrm{Et}_{2} \mathrm{O}, \mathrm{MeOH}-\mathrm{CH}_{2} \mathrm{Cl}_{2}}
$$<smiles>[R]C(N)C(C)=O</smiles>

270

$\mathrm{R}=\mathrm{PhO}\left(\mathrm{CH}_{2}\right)_{4}-(50 \%) ; \mathrm{CH}_{3} \mathrm{OCO}\left(\mathrm{CH}_{2}\right)_{2}-(49 \%) ; c-H e x(44 \%) ; 1$-adamantyl (39\%)

4-t-Bu- $\mathrm{PhCH}_{2}-(22 \%) ; \mathrm{Bn}(25 \%) ; \mathrm{R}^{\prime} \mathrm{OCOCH}_{2}-$ (no reaction). Yields calculated on 3 steps.

The reaction was successful for unactivated alkyl radicals, lower-yielding in the case of addition of benzyl radicals (either from benzyl iodide or bromide), while no reaction was observed for $\alpha$-carbonyl radicals, which suggests that the process is governed by radical philicity.

\subsection{Carbon-Carbon Bond Formation through Intramolecular Reactions}

\subsubsection{Radical Addition to Oximes}

In successive papers [96,97,101-103], Naito's group continued to study the reactivity of oxime ethers on solid-support by testing a 5-exo-trig radical cyclization for the synthesis of pyrrolidines (Schemes 92 and 93).

Scheme 92. Naito's SP-atom transfer radical cyclization into oxime-alkene.

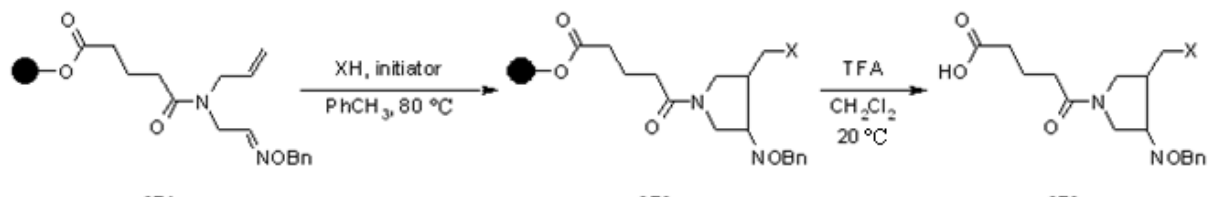


Scheme 93. Naito’s SP-atom transfer radical cyclization into oxime-alkyne.

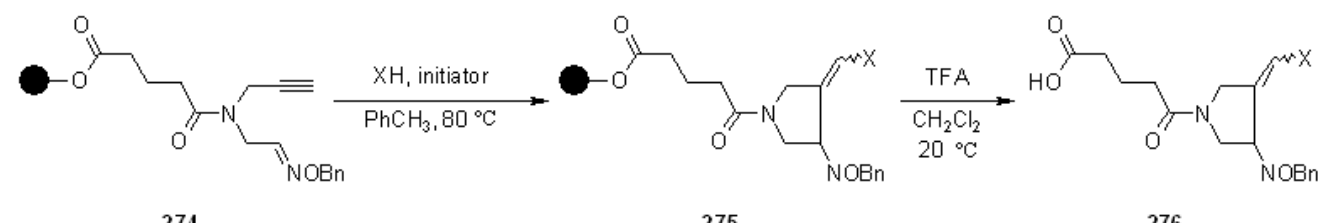

274

275

276

Resins 271 (Scheme 92) and 274 (Scheme 93) were prepared and reacted to test their cyclization potentiality. The major difference between the reactions depicted in Schemes 89-91 was failure of cyclization at low temperature. For this reason, it was necessary to use toluene as solvent, heated up to $80{ }^{\circ} \mathrm{C}$. The use of $\mathrm{Bu}_{3} \mathrm{SnH}$ as $\mathrm{XH}$ gave reasonable yield when AIBN was used as radical initiator (Scheme 92: 47\%). Better results were obtained using $\mathrm{Et}_{3} \mathrm{~B}$ (Scheme 92: 64\%; Scheme 93: 77\%), while further variation of the radical initiator did not improve the process: in fact, 9-BBN gave only trace of product and $\mathrm{Et}_{2} \mathrm{Zn}$ gave successful cyclization, but low yield (Scheme 92: 26\%). Use of TTMSS in place of $\mathrm{Bu}_{3} \mathrm{SnH}$ gave lower yield (Scheme 92: 50\%). As observed previously (see Scheme 89), the desired reaction is always in competition with ethyl radical addition-cyclization and when a less reactive radical precursor such as $\mathrm{Et}_{3} \mathrm{SiH}$ was used, the sole product was $\mathrm{X}=\mathrm{Et}$. The same reaction was successfully tried on an appropriate substrate containing a solid-supported $\alpha, \beta$-unsaturated amide (Scheme 94) and extended to check the possibility of stereoselection using an asymmetric substrate (Scheme 95).

Scheme 94. Radical addition to an electron-poor alkene.

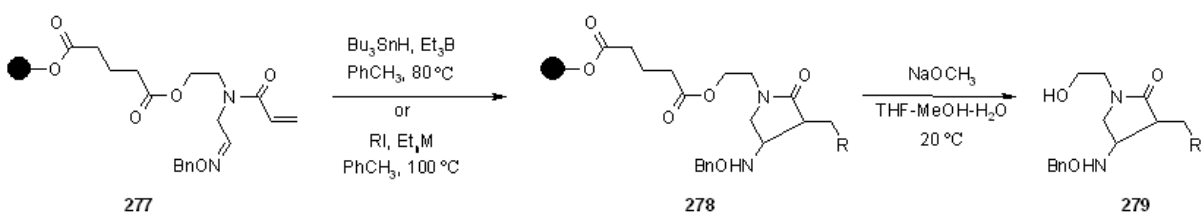

Scheme 95. Example of stereoselective addition/cyclization reaction.

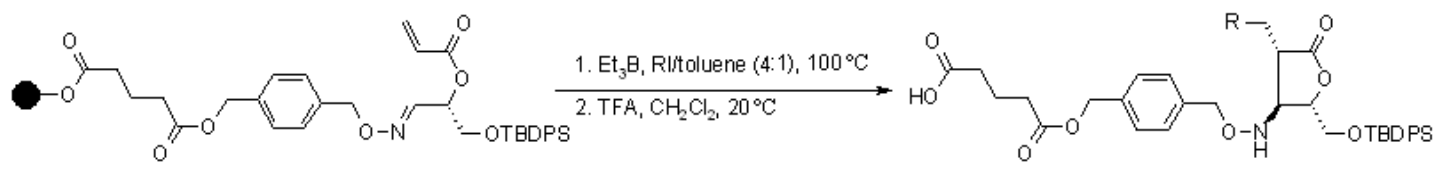

280

281

The radical addition to an electron-poor alkene is shown in Scheme 94. In a first attempt the addition of a stannyl radical was tested and product $\mathbf{2 7 9}$ was recovered after cleavage in good yield (64\%). The same reaction was then run under iodine-atom transfer conditions, using different R-I. The results were satisfying, although a large amount of alkyl iodide had to be used (30 eq.). The only reaction that failed was the one with the bulky $t$-Bu radical ( $\mathrm{R}=i$-Pr: $69 \%$; $c$-hexyl: 54\%; $c$-pentyl: 59\%; $s$-Bu: 55\%). The results obtained using $\mathrm{Et}_{\mathrm{n}} \mathrm{M}$ without $\mathrm{R}-\mathrm{I}$ were rather interesting, thus, when $\mathrm{Et}_{3} \mathrm{~B}$ was used, a $72 \%$ of 279 product $(\mathrm{R}=\mathrm{Et})$ was isolated, while the use of $\mathrm{Et}_{2} \mathrm{Zn}$ gave only a $12 \%$ of the same product.

Scheme 95 displays the stereoselective addition/cyclization reaction promoted by alkyl radicals, using asymmetric substrate $\mathbf{2 8 0}$. The reaction was run in a strong excess of R-I ( $4: 1 \mathrm{v} / \mathrm{v}$ with toluene), because, with smaller amounts, the product of ethyl radical addition was predominant. The products 
281 were recovered in reasonable yields ( $\mathrm{R}=i$-Pr: 57\%; $c$-hexyl: 50\%; Et: 92\%, when the reaction was run with only $\mathrm{Et}_{3} \mathrm{~B}$ ), but with a constant diasterometic ratio of $8: 1$, independently of the nature of the adding radical.

\subsubsection{Radical Addition to Alkenes, Alkynes and Arenes}

A radical cyclization for the synthesis of 2-oxindoles was studied by Fukase's group [104]. In this case, $\mathrm{N}$-(2-bromophenyl)acrylamides were anchored to the $\mathrm{SP}$ and treated under classical radical conditions $\left(\mathrm{Bu}_{3} \mathrm{SnH}, \mathrm{AIBN}\right)$ (Scheme 96).

Scheme 96. Fukase's SP-radical cyclization into alkenes.<smiles>[R3]C=C([R4])C(=O)N(Cc1ccc(C(=O)NC)cc1)c1c([R])cc([R1])cc1Br</smiles>

282

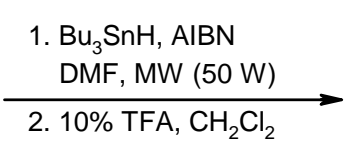

2. $10 \%$ TFA, $\mathrm{CH}_{2} \mathrm{Cl}_{2}$

40 derivatives: yields: $66-100 \%$<smiles>[R3]CC1([R4])C(=O)N(Cc2ccc(C(N)=O)cc2)c2c([R2])cc([R1])cc21</smiles>

283

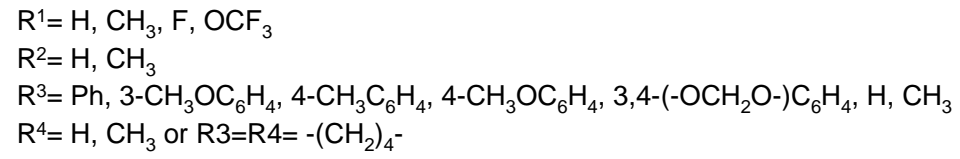

Oxindoles such as $\mathbf{2 8 3}$ were obtained smoothly under MW irradiation $(<1 \mathrm{~h})$, compared to conventional heating $(24 \mathrm{~h})$. Interestingly, the reaction was run in $\mathrm{DMF}$, a solvent where $\mathrm{Bu}_{3} \mathrm{SnH}$ is rather insoluble. This characteristic forced an increase in concentration of the $H$-donor on the surface of the resin. In addition, the same reaction run in solution gave no product at all, sustaining the thesis of concentration effect [105].

Bowman and coworkers [106] studied the addition of reactive aryl radicals to azoles and extended the methodology to SP using less toxic and troublesome TBGH and TTMSS (Scheme 97).

Scheme 97. Bowman's SP-radical addition into pyrazole.

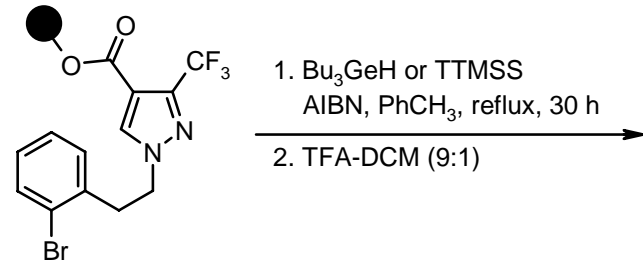

284<smiles>O=C(O)c1c(C(F)(F)F)nn2c1-c1ccccc1CC2</smiles>

285<smiles>[NH3+][C@@H](c1nn(CCc2ccccc2Br)cc1C(=O)O)C(F)(F)F</smiles>

286<smiles>O=C(O)c1cn(CCc2ccccc2)nc1C(F)(F)F</smiles>

287 
The probe scaffold was anchored to a Wang resin. The results were rather disappointing compared to the relative reactions carried out in solution: in fact, when TBGH was used only $20 \%$ of $\mathbf{2 8 5}$ was isolated after cleavage, together with $70 \%$ of unreacted 286. However, switching to TTMSS improved the yield of 285 (53\%), but this time, the side product was the reduced, uncyclized $287(27 \%)$.

De Maesmaeker and Wendeborn [107] studied the functionalization of cyclohexendiol derivatives via radical cyclization on solid phase. Cyclization was performed using an aryl radical (Scheme 98) and a vinyl radical (Scheme 99), giving in both cases a mixture of products.

Scheme 98. De Maesmaeker's SP-aryl radical cyclization into alkene.

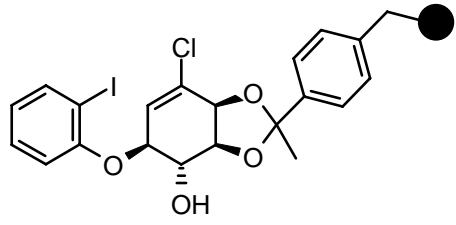

288

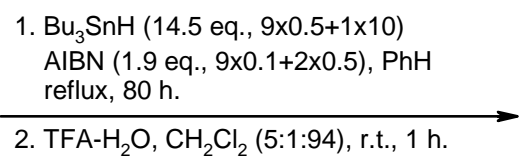

2. TFA- $\mathrm{H}_{2} \mathrm{O}, \mathrm{CH}_{2} \mathrm{Cl}_{2}(5: 1: 94)$, r.t., $1 \mathrm{~h}$<smiles>[X]C1[C@H](O)[C@H](O)[C@H](O)[C@H]2Oc3ccccc3[C@H]12</smiles>

289

a: $X=\alpha+\beta-\mathrm{Cl}(20 \%)$ b: $\mathrm{X}=\mathrm{H}(51 \%)$<smiles></smiles>

$290(20 \%)$

Scheme 99. De Maesmaeker's SP-vinyl radical cyclization into alkene.

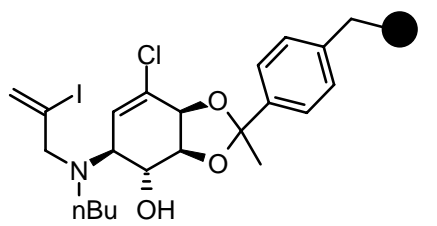

291

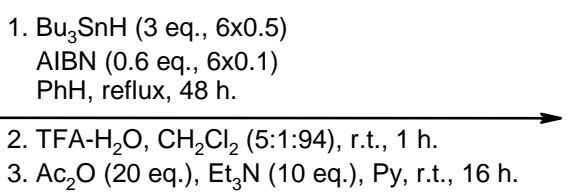

3. $\mathrm{Ac}_{2} \mathrm{O}$ (20 eq.), $\mathrm{Et}_{3} \mathrm{~N}$ (10 eq.), Py, r.t., 16 h.

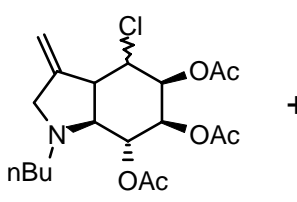

$292(52 \%)$

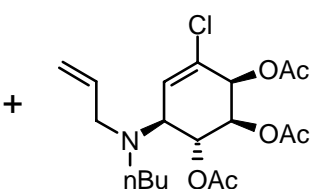

$293(40 \%)$

In the first case (Scheme 97), product 289a was obtained as the minor component together with the prematurely reduced starting material 290, and the product of over-reduction of 289a $(\mathbf{2 8 9} \mathbf{b}, \mathrm{X}=\mathrm{H})$. Unfortunately, it resulted impossible to achieve complete removal of the chlorine atom without increasing the amount of $\mathbf{2 9 0}$. In the second reaction (Scheme 98), a large amount of reduced product 293 was found, while chlorine atom resisted in the desired product 292.

$\mathrm{Du}$ and Armstrong [108] provided a milder approach to the synthesis of solid-supported benzofurans via radical cyclization, which is based on the use of $\mathrm{SmI}_{2}$ (Scheme 100).

Scheme 100. Armstrong's SP-aryl radical cyclization into alkenes.

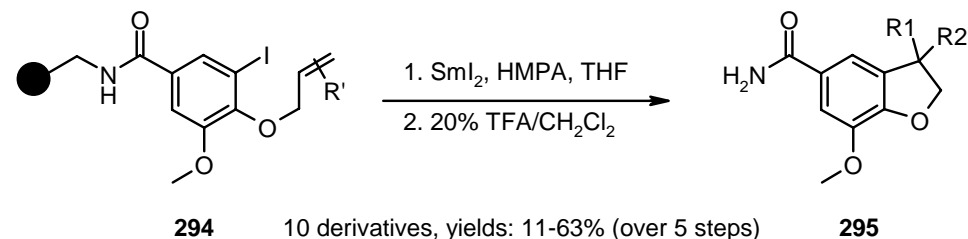

Yields were measured over 5 steps (4 of them necessary to synthesize 294). The reaction was run at room temperature and a range of diverse substituted alkenes was tested. The cyclization reaction took place in almost all cases, being the $\alpha, \beta$-unsaturated ester (294 with $\mathrm{R}^{\prime}=\mathrm{COOEt}$ ) derivative an exception. However, in some cases, the yields were lower due to a variable amount of 
disproportionation product. This side-reaction proved to be substrate-dependent more than SP-dependent, since parallel test reactions in solution were carried out, revealing similar problematic. A major drawback of this approach could be the use of toxic reagents in excess (HMPA was necessary for the reaction to take place) and the difficulty in washing out the samarium ions when ungrafted polystyrene resin (Rink) was used. The latter problem was resolved using polyethylene glycol grafted resin, which swells well in aqueous solvents, making wash-up with $\mathrm{NaHCO}_{3}$ saturated solution more efficient for the elimination of $\mathrm{Sm}^{3+}$ impurities from the beads. In a subsequent paper [109], the same group brought forward the reaction depicted in the previous scheme by capture of the anionic metal derivative, formed after cyclization, with an electrophile (Scheme 101).

Scheme 101. Example of radical cyclization/electrophile capture.

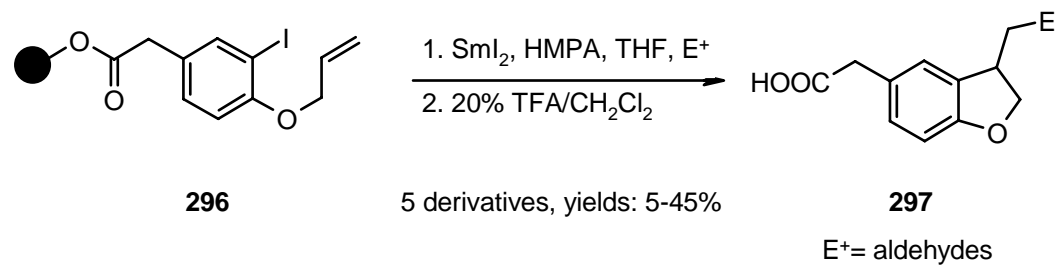

The electrophiles chosen were aldehydes, using reaction conditions similar to those described earlier. As reference, the test reactions were also run in solution, where it was acknowledged the short half-life of the anion formed.

Compared to the previous example, attachment to the SP was performed via an appropriate handle on the benzene ring. In a first attempt Rink resin was used, giving no product of electrophilic quenching of the anion. Since the amide nitrogen in the linking group of Rink was supposed to be responsible for early interception of the anion generated after radical cyclization, it was decided to use the ester linkage of TentaGel S BHP resin (a resin also swelling better in the solvent used for the reaction). In this case, interception was effective, even if in a mixture with simple cyclized product. Since the yields were strongly dependent on the electrophile nature, this method did not present the quality necessary to be used in combinatorial chemistry.

Following the same idea, Berteina and De Maesmaeker [110] tested the 5-exo-trig cyclization to form 2,3-dihydro-benzofurans. Either the aryl iodide or the unsaturated counterpart, tethered by means of an oxygen atom, was directly anchored to aminomethylphenyl functionalized polystyrene beads. Usually, a spacer was inserted between the resin and the reactive molecule. As first attempt, the radical cyclization was performed under usual radical conditions (Scheme 102).

Scheme 102. De Maesmaeker's SP-aryl radical cyclization into alkenes.

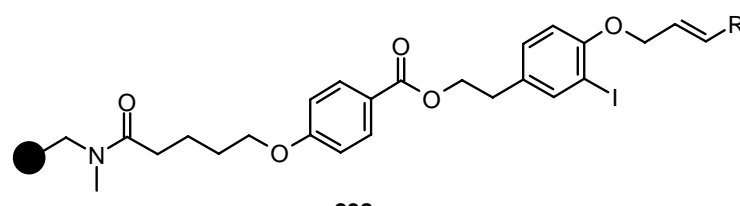

298

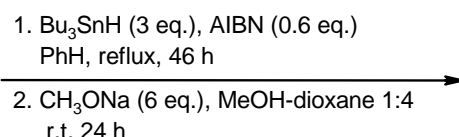

r.t. $24 \mathrm{~h}$<smiles>OCCc1ccc2c(c1)C(CO)CO2</smiles>

299

$\mathrm{R}^{1}=\mathrm{H}, 97 \%$ $\mathrm{R}^{1}=\mathrm{CH}_{3}, 95 \%$ $\mathrm{R}^{1}=\mathrm{Ph}, 95 \%$ 
The radical cyclization depicted in Scheme 102 was effective, high yielding and no product of early reduction or starting material was found.

Interception of the alkyl radical formed after cyclization with allytributyltin was also attempted (Scheme 103). For $\mathrm{R}^{1}=\mathrm{H}$, the reaction gave a good yield of desired product $\mathbf{3 0 0}$ (beside $22 \%$ of simple cyclized product 299), while for $\mathrm{R}^{1}=\mathrm{CH}_{3}$ or $\mathrm{Ph}$, yields were pretty low and after cleavage the main product was found to be the unreacted starting material (beside $10 \%$ of 299), demonstrating a slower reaction that can be explained on the basis of steric hindrance.

Scheme 103. Example of radical cyclization/allyl interception.

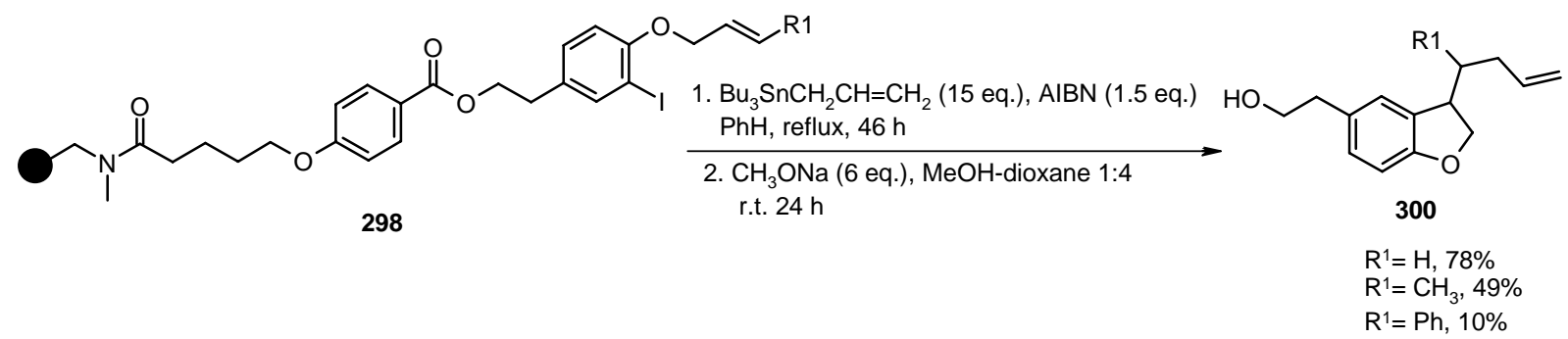

In a second attempt, it was chosen to have the unsaturated moiety, in this case an alkyne, directly connected to the SP. The reaction led to a mixture of two products 302 and 303 (Scheme 104).

Scheme 104. De Maesmaeker's SP-aryl radical cyclizatino into alkyne.

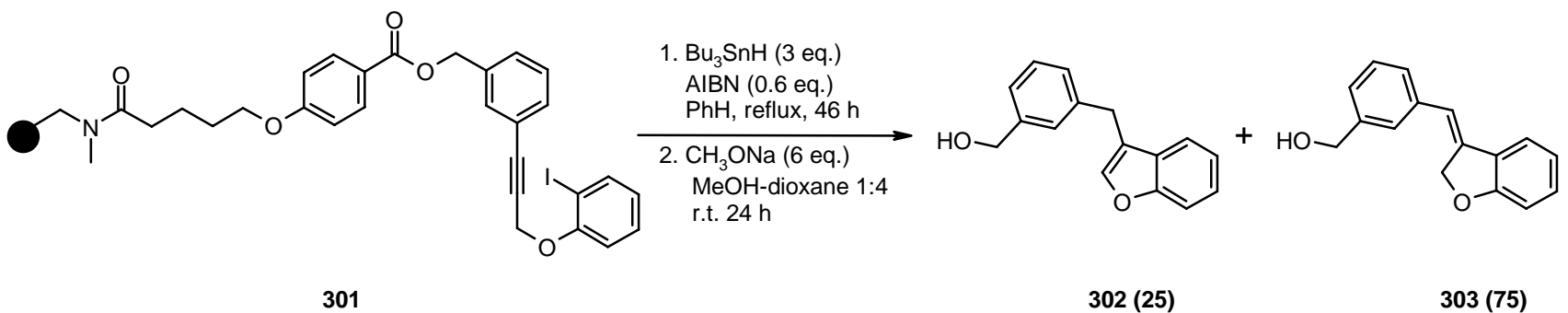

In another publication [111], the same group used a similar 5-exo-trig cyclization to form 1,3dihydro-isobenzofurans (Scheme 105).

Scheme 105. De Maesmaeker's synthesis of benzofurans 305.

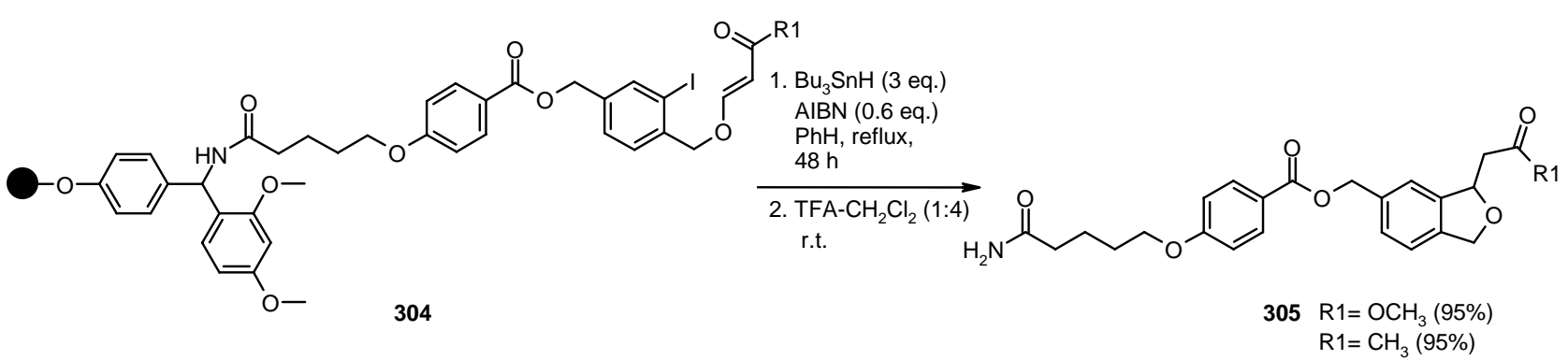


The acid-labile Rink linker was necessary since, following the strategy adopted in Schemes 103 and 104, low yields of desired products (due to partial decomposition under the basic cleavage conditions) were obtained. This change of plan gave almost quantitative yields after cleavage.

In another experiment, it was evaluated the radical cyclization onto an aromatic ring, which yielded a benzoquinoline (Scheme 106).

Scheme 106. De Maesmaeker's SP-aryl radical cyclization into a benzene ring.

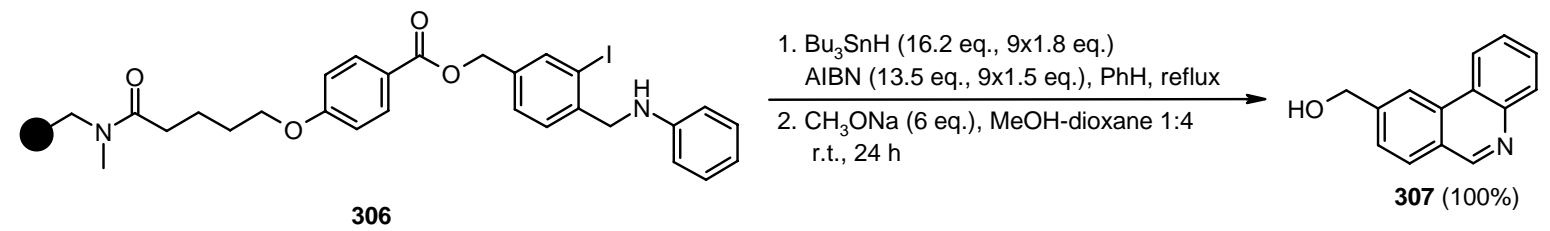

In this case, it was necessary to use large amounts of $H$-donor and, most importantly, of radical initiator (AIBN), added in aliquots during the reaction. Lower amounts of the reagents gave uncompleted conversions. The need of a large excess of AIBN is probably due to its role played in the aromatization step after cyclization.

Also Routledge's group [112] used the intramolecular radical cyclization to form tetrahydrofurans derivatives (such as the one depicted in Scheme 100) testing the use of hypophosphite salts as radical carriers in alternative to the toxic TBTH on SP (Scheme 107).

Scheme 107. Routledge's SP-aryl radical cyclization into alkene.

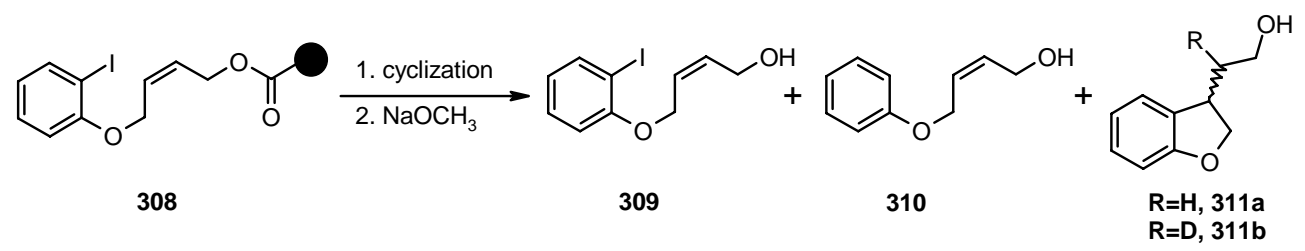

As reference, the reaction was first run using TBTH (10 eq., toluene, reflux, $4 \mathrm{~h}$ ) and AIBN ( 1 eq.), on a $2 \%$ divinylbenzene cross-linked carboxypolystyrene resin, giving a $95 \%$ yield of cyclized product 311a (beside $4 \%$ of 309 and 1\% of 310). The reaction was then run using $N$-ethylpiperidine hypophosphite (EPHP, 20 eq.) and AIBN (3 eq.), but at best, only a 57\% yield of 311a was isolated (besides $38 \%$ of $\mathbf{3 0 9}$ and $5 \%$ of $\mathbf{3 1 0}$ ). The reason for this behavior was initially supposed to lay in the nature of the solvent used (toluene), since EPHP is a salt and its concentration on the surface of the resin could be low in apolar solvents. However, when the reaction was run in polar mixtures (THF-EtOH 4:1), no real change of product distribution was observed (63\% 311a, 31\% 309, 6\% 310). The attention was then shifted to the nature of the solid phase and a range of cross-linked and PEG grafted solid-supports was tested. The idea was that resins more hydrophilic than polystyrene would allow the radical carrier to approach the solid surface. For the purpose the following resins were used: NovaSyn $^{\circledR}$ TG carboxy resin, a macroporous resin (ArgoPore ${ }^{\circledR}$ ), PEG grafted resins, HypoGel ${ }^{\circledR}$ and ArgoGel $^{\circledR}$ and the polytetrahydrofuran cross-linked resin, JandaJel ${ }^{\circledR}$. The best results were obtained using the latter, which gave a $98 \%$ of 311a (beside a $2 \%$ of 310), under optimized conditions (20 eq. 
EPHP, initiator 2 eq., THF-EtOH 4:1, 48 h, under reflux). To confirm the validity of the choice made, a different reaction was carried out, using a primary alkyl radical precursor 312 (Figure 108).

Figure 108. Structure of alkyl radical precursor 312.

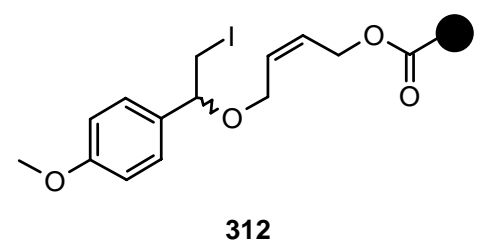

The reaction was successful, with a $92 \%$ yield of desired cyclized product. The use of alternatives such as EPHP on SP could allow radical chemistry to become a more useful method for SP synthesis, with potential extension to combinatorial and medicinal chemistry.

In a recent paper [113], the same group studied the influence of microenvironment effects in the reaction depicted in Scheme 107 by determination of deuterium incorporation in the cyclized product. In order to do so, cyclization was carried out using deuterated EPHP or $\mathrm{Bu}_{3} \mathrm{SnD}$ in deuterated solvents ( $\mathrm{d}_{8}$-THF: $\mathrm{d}_{6}-\mathrm{EtOH} 4: 1$ or $1: 1 ; \mathrm{d}_{4}-\mathrm{MeOH} ; \mathrm{d}_{6}$-benzene). The ratio of deuterated product $311 \mathrm{~b}(\mathrm{R}=\mathrm{D})$ versus 311a $(\mathrm{R}=\mathrm{H})$ was measured by $\mathrm{GC}-\mathrm{MS}$ analysis.

The objective was the rationalization of the role played by the polymer backbone/linker in the radical $H$-abstraction, a side-reaction encountered in radical chemistry on SP. A first conclusion was that, as already observed by Curran [8], all resins can compete with relatively slow $H$-transfer reactions (such as $H$-abstraction from EPHP by an alkyl radical). However, the overall picture indicated that there are multiple factors influencing radical chemistry on SP: nature of the resin and linker (flexibility), solvent (swelling properties) and nature of the radical carrier (bond strength and solubility). Therefore, a general prediction of the effects of a specific resin on kinetics and mechanism of a radical reaction is rather difficult and the combination of the above-mentioned factors need to be considered case-by-case.

A more traditional approach was used by Balasubramanian and coworkers [114], who performed aryl radicals cyclization into alkenes to prepare benzofuran derivatives (Scheme 109) and alkyl radicals cyclization into alkynes to prepare functionalized furans (Scheme 110). The reactions were run using a very large excess of the $H$-donor, $\mathrm{Bu}_{3} \mathrm{SnH}$. When the same excess of organostannyl hydride was used in solution, large amount of early reduction products were normally obtained. Therefore, somehow, use of the solid phase seemed to neutralize this inconvenience.

Scheme 109. Balasubramanian's SP-aryl radical cyclization into alkene.<smiles>CC(=O)OC/C=C/COc1ccc(N)cc1Br</smiles>

313

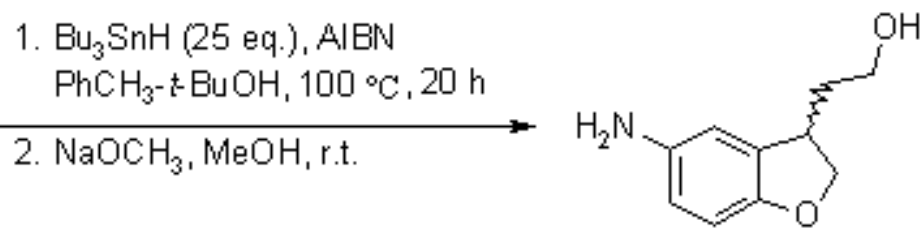

314 
Scheme 110. Balasubramanian's SP-alkyl radical cyclization into alkene.

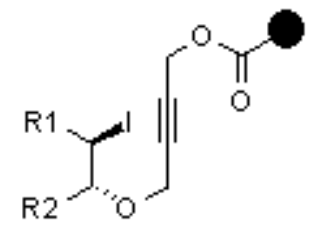

315

$\mathrm{R}^{1}=\mathrm{Ph}_{1} \mathrm{t}-\mathrm{Bu} ; \mathrm{R}^{2}=\mathrm{H}$

or

$\mathrm{R}^{\prime}=\mathrm{R}^{2}=-\left(\mathrm{CH}_{2}\right)_{3},-\left(\mathrm{CH}_{2}\right)_{4}$

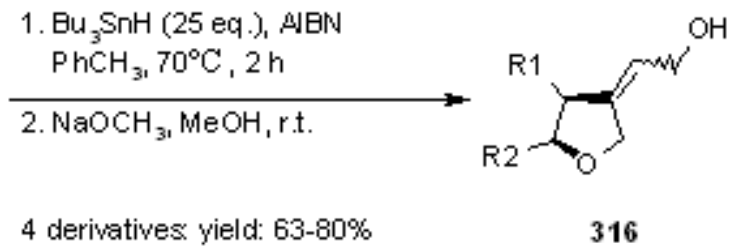

4 derivatives yield: $63-80 \%$

Both carboxylated PS and TentaGel-COOH were used as resins. The main difference among them was the amount of radical initiator needed; in fact, in the former case, an excess of AIBN had to be used, while in the latter, only $6 \%$ mol was sufficient to perform complete conversion. The reason for this different behavior was attributed to chain-terminating processes involving the benzylic hydrogen on the polystyrene backbone, absent in the TentaGel resin. The lack of reduced side-products was tested on a slower (three orders of magnitude) reaction (shown in Scheme 110) usually more sensitive to early reduction, but even in this case it was not observed.

In an application to the synthesis of molecule of biological interest, a 5-exo-trig radical cyclization was performed by Lown's group [115] for the synthesis of 1-chloromethyl-1,2-dihydro-3Hbenz[e]indole (seco-CBI) and a polyamide conjugate (Scheme 111).

Scheme 111. Lown's use of SP-aryl radical cyclization for the synthesis of seco-CBI.

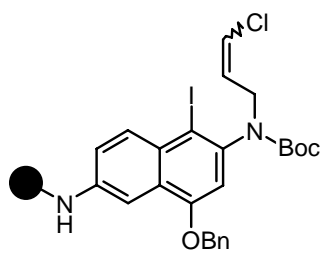

317

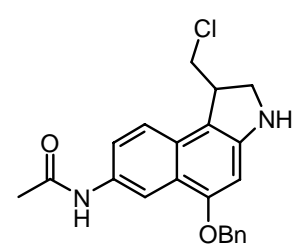

318

The radical reaction was carried out starting from bromo-Wang resin according to the procedures applied in solution, commonly used for the synthesis of CC-1065 derivatives. To measure the efficacy of the reaction on SP, the product of cyclization was first acetylated and then cleaved, to yield a total $74 \%$ ( $48 \%$ plus a $26 \%$ of the product with the Boc protecting group still in place). The synthesis of a bis-polyamide-seco-CBI conjugate was completed using $\mathbf{3 1 8}$ and performing additional amide couplings both on solid- and solution-phase. Toru and colleagues [116] performed the intramolecular radical addition of an alkyl radical to unsaturated $C$ - $C$ bonds, in order to form $\gamma$-butyrolactones (Scheme 112).

Scheme 112. Toru's SP-alkyl radical cyclization into alkenes.

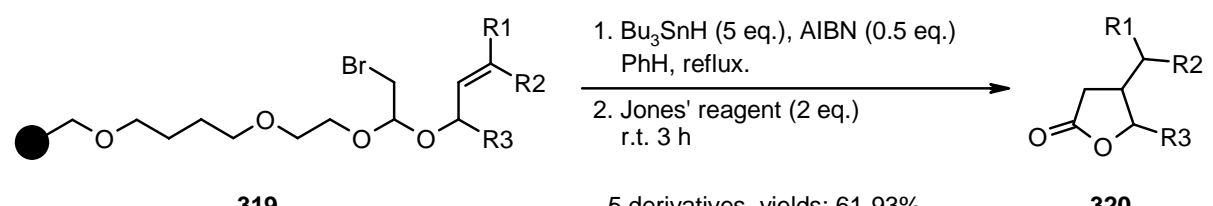


Merrifield resin was functionalized with an appropriate linear spacer and radical precursors 319 were prepared on the SP by addition of allyl (or propargyl) alcohols to the loaded vinyl ether, in the presence of NBS. The radical reaction was performed under common conditions and the excess of H-donor did not give uncyclized, reduced side-products. The toxic organotin by-products were separated by filtration and washing, while cleavage of the desired products from the resin was achieved using Jones' oxidation. Yields were rather satisfactory, even when a 5-exo-dig cyclization on a triple bond was tested $(47 \%, E: Z=1: 9)$.

During his studies towards the use of supported radical chemistry, Enholm [117] proposed the first example of stereoselective radical cyclization on a soluble support. The use of soluble supports (precipitating at $-78{ }^{\circ} \mathrm{C}$ in $\mathrm{MeOH}$ ) represent a great advantage for SP radical reactions since, according to the authors, the rates of the reactions involved are similar to those run in solution. Thus, a custom polymer incorporating a chiral auxiliary unit was synthesized by ring opening metathesis polymerization (ROMP). The radical reaction studied was a 6-exo-trig cyclization (Scheme 113).

Scheme 113. Enholm's stereoselective alkyl radical cyclization on ROMP polymer.

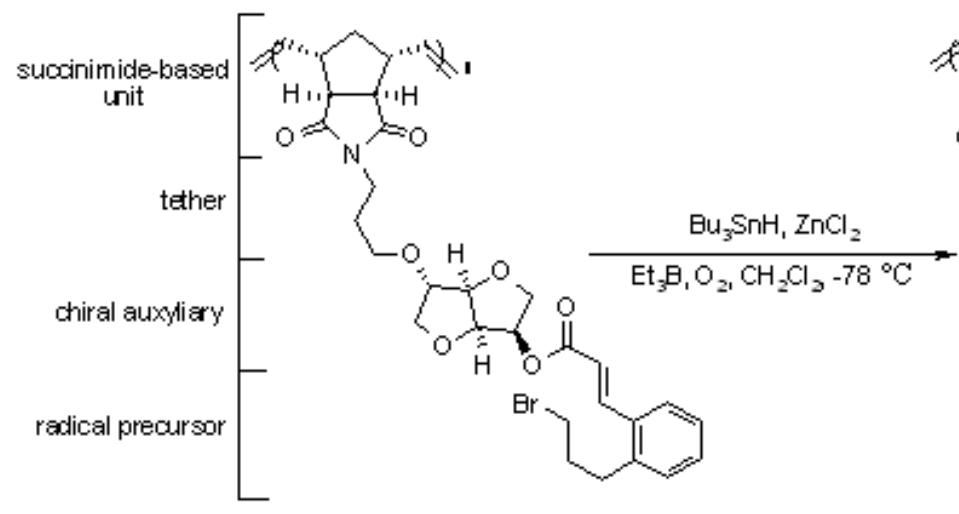

321

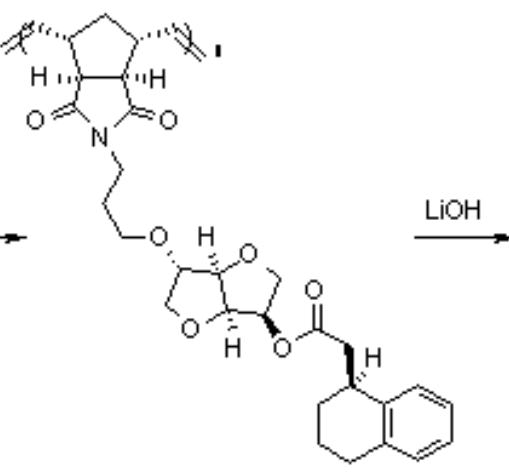

322

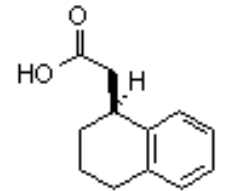

323

After building the monomer, the polymer was prepared employing a Grubbs-type ruthenium-based catalyst. Each monomer contained a succinimide anchor tethered to an $(+)$-isosorbide unit (the chiral auxiliary) esterificated with the radical precursor. After the ROMP, the polymer obtained reacted under radical conditions in the presence of a Lewis acid. Subsequent cleavage with lithium hydroxide afforded the desired acid 323. Constructing the polymer in this way, it was possible to achieve a $100 \%$ loading, a result not viable using commercial resins. The results were encouraging since, beside an $80 \%$ isolated yield, there was a $>99 \%$ ee (being $\mathrm{ZnCl}_{2}$ the best solution). The use of a combination of low temperatures and Lewis acid for stereoselective radical cyclization is known [118-123], however, in this case, the use of zinc chloride proved to be far more better than other LA (such as $\mathrm{MgBr}_{2}$ or $\left.\mathrm{Yb}(\mathrm{OTf})_{3}\right)$, probably owing to the presence of the polymer-embedded sugar which creates a favorable environment for zinc-mediated stereoselection.

Kilburn's group [124] exploited the reactivity of isocyanides to perform thiol-mediated radical cyclizations to obtain pyrroline or pyrrolidinone derivatives. In Scheme 114 a Wang-type resin was used. Treatment under radical cyclization conditions in the presence of 2-mercaptoethanol gave resin 325 that after trifluoroacetic acid-mediated cleavage gave pyrrolidinone $\mathbf{3 2 6}$ in reasonable yields. 
Scheme 114. Kilburn's thiol-mediated radical cyclization into SP-isocyanides.

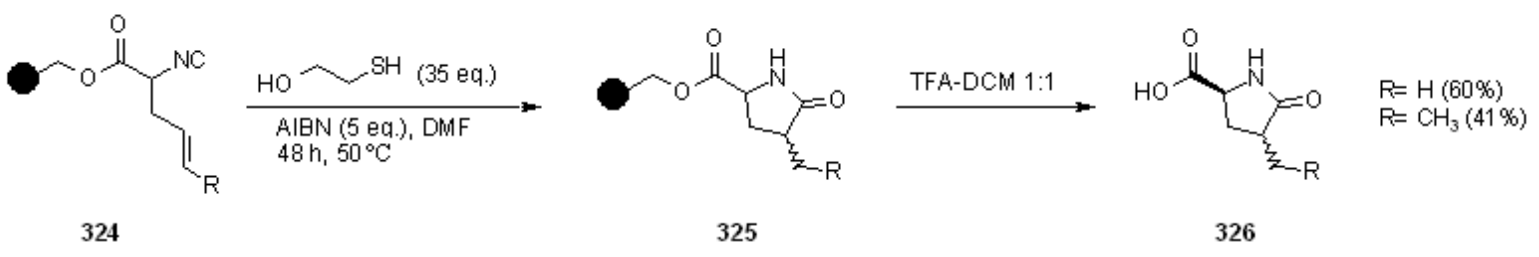

When HMBA-AM resin was used (Scheme 115), the use of ethanethiol during the cyclization step gave rise to resin 328, which could be cleaved under nucleophilic conditions preserving the pyrroline structure to yield amide derivative 329. In both cases the starting isonitrile was built on resin, which has the advantage of avoiding the handling of the unpleasant substance.

Scheme 115. Kilburn's pyrroline synthesis.<smiles>C=CCC(C)C(=O)OCc1ccc(C(=O)NCC)cc1</smiles>

Harrowven and colleagues [125] reported two new methods for radical cyclization on SP using supported dienes and, respectively, thiyl and tosyl radical. In this case heating of the loaded resin in the presence of AIBN and the radical precursor $(\mathrm{PhSH}$ or $\mathrm{TsSePh})$ in an appropriate solvent was sufficient (Scheme 116).

Scheme 116. Harrowven's SP-atom transfer radical cyclization into alkenes-alkenes.

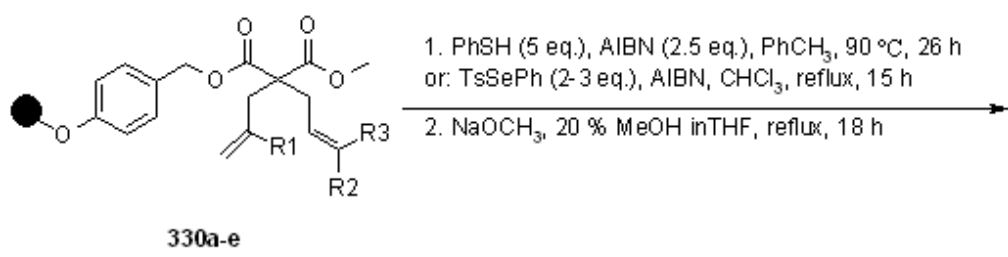

Radical precursors 330a-e were loaded on a PS-Wang resin. The results obtained when using phenylthiol $(\mathrm{X}=\mathrm{S}, \mathrm{Y}=\mathrm{H})$ as radical source/cyclization promoter were rather satisfactory, with yields of 331 ranging between $69-78 \%$; when using $p$-tolyl benzeneselenosulfonate $(\mathrm{X}=\mathrm{Se}, \mathrm{Y}=\mathrm{Ts}$ ) on the same substrates, the desired products 332 were recovered in comparable yields (66-74\%). The isomeric product distribution followed Beckwith's guidelines for radical cyclizations [126,127].

\subsection{Carbon-Heteroatom Formation}

An interesting radical cyclization initiated by IBX was discovered [128-130] and thoroughly studied by Nicolaou. Mechanism and limits of the reaction as well as an application in solid-phase were subsequently reported [131] (Scheme 117). The interesting mechanism comprises a radical reaction initiated by IBX (A), where THF actively participate (B) and explains the reason for the need of an 
aryl amide for the success of the reaction. While it was tested and performed with good results in solution, the extension to the SP resulted in poor yield of the desired product (15\%), probably due to oxidation of the resin by the IBX excess.

Scheme 117. Nicolaou's IBX-promoted SP-radical cyclization.

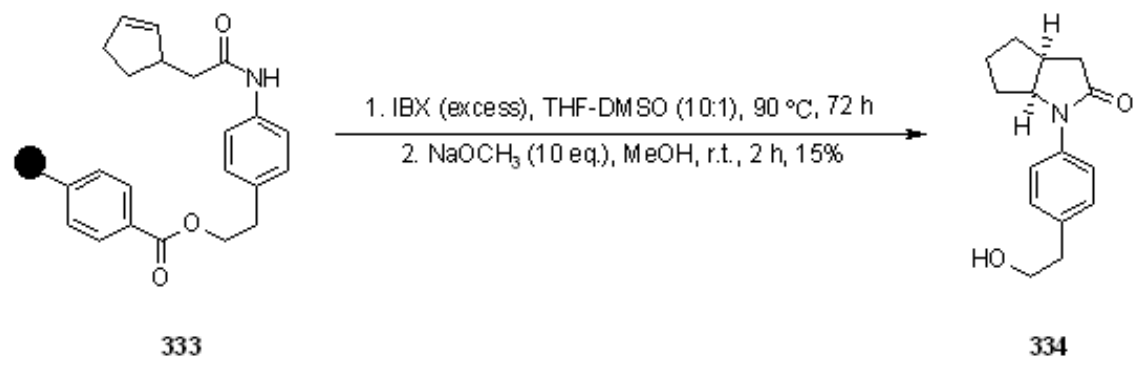

Propasedmechanism:
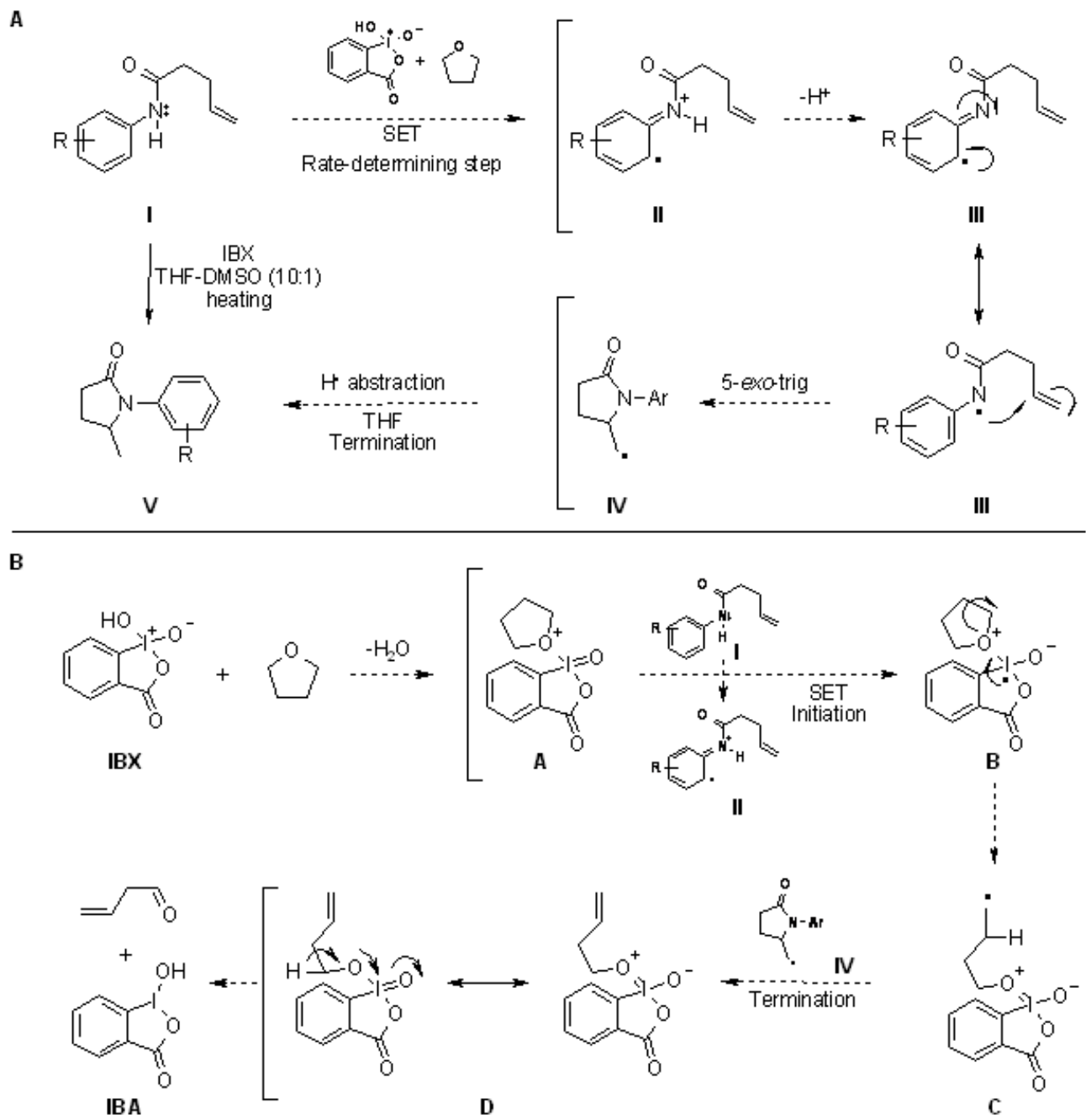

Caddick and coworkers performed the addition of sulfonyl radicals to isolated alkenes (Scheme 118) and alkynes (Scheme 119) [132] and the best conditions are displayed below. Interestingly, the nature of solvent, linker (eq. 1,2 of Scheme 118 vs. eq. 1,2 of Scheme 119) and resin did have great influence on the reaction outcome, spanning between combinations were there was no reaction at all, to conditions giving decomposition. 
Scheme 118. Caddick's radical addition to alkynes.
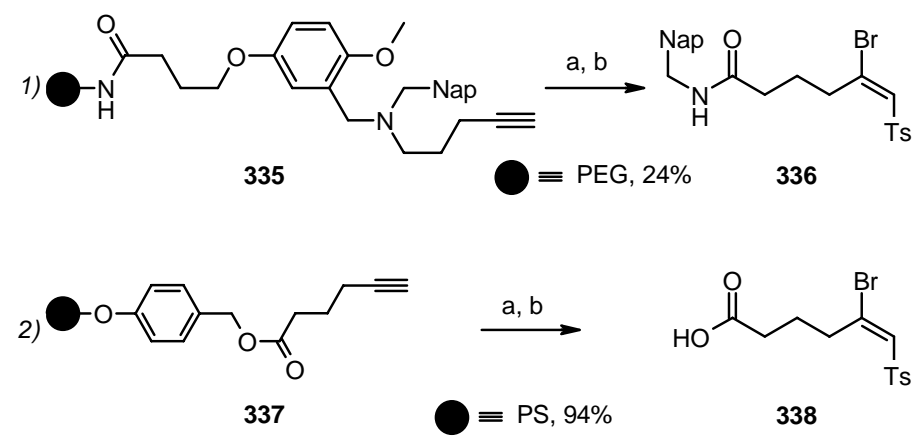

a) $\mathrm{TsBr}, \mathrm{AlBN}, \mathrm{PhCH}_{3}, 65-70{ }^{\circ} \mathrm{C}$; b) $95 \% \mathrm{TFA}$ (aq.)

Scheme 119. Caddick's radical addition to alkenes.

1)

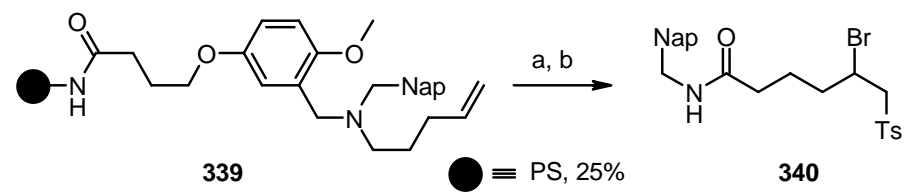

2)

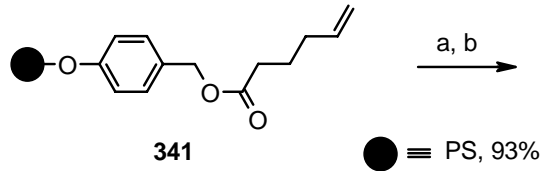<smiles>O=C(O)CCCC(Br)C[123I]</smiles>

342

a) $\mathrm{TsBr}, \mathrm{AlBN}, \mathrm{PhCH}_{3}, 65-70{ }^{\circ} \mathrm{C}$; b) $95 \%$ TFA (aq.)

During the studies about the transformation of hydrophilic to hydrophobic groups, in order to change the pharmacological properties of molecules, Plourde Jr. [133] explored the intermolecular radical addition of thiols to supported alkenes (Schemes 120 and 121).

Scheme 120. Plourde Jr.'s radical addition to supported alkenes.

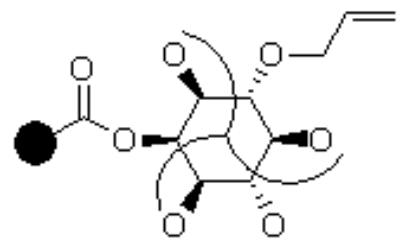

343

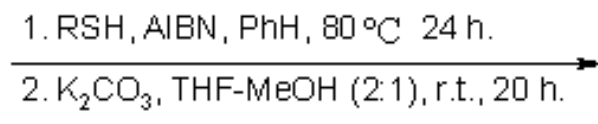

4 derivatives: yields: $68-87 \%$

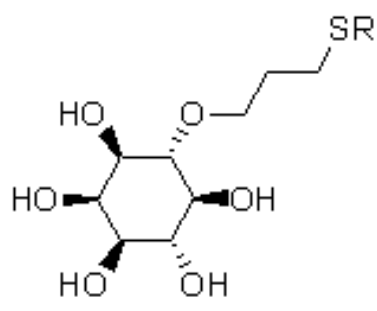

344

$\mathrm{R}=c-p e n t y(68 \%) ; \mathrm{Bn}(85 \%) ; \mathrm{CH}_{2}(\mathrm{OH}) \mathrm{CH}(\mathrm{OH}) \mathrm{CH}_{2}-(87 \%) ; \mathrm{HO}_{2} \mathrm{CCH}_{2}-(83 \%)$

The reactions were run under the usual radical conditions, even if when the carbamate linker was used (Scheme 121) an uncommon solvent for radical reactions such as DMF was chosen. Yields were generally good, however, when $\mathrm{R}=$ Ar or HetAr, no reactions was observed (not shown). Addition of a thiol-bearing resin to an alkene was employed also for the synthesis of a new linker useful for the synthesis of amines. In order to improve the scope of the 2-(thiobenzyl)ethyl carbamate linker normally used for such a purpose, Timár and Gallagher [134] proposed to start from Merrifield SH resin. 
Scheme 121. Example of Plourde Jr.'s use of carbamate linker.

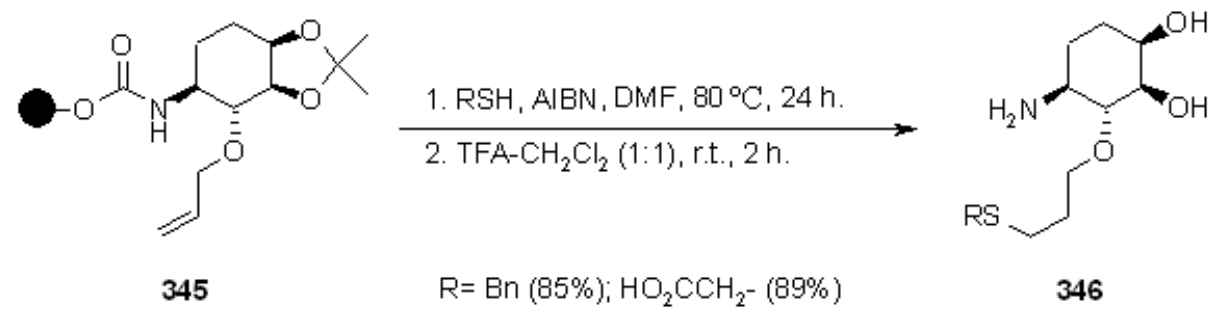

This (Scheme 122) was treated with the previously prepared $N$-vinyloxycarbonyl derivative 348, under radical conditions. Addition of the benzyl thiyl radical to the vinyloxy moiety loaded the desired starting material to the resin, giving 349, which was treated under oxidative/basic conditions for cleavage. This modification offers an entry for secondary amines, complementing the original procedure (exploiting the reaction between a supported alcohol and an isocyanate), only viable for primary amines.

Scheme 122. Gallagher's SP-radical synthesis of amines.

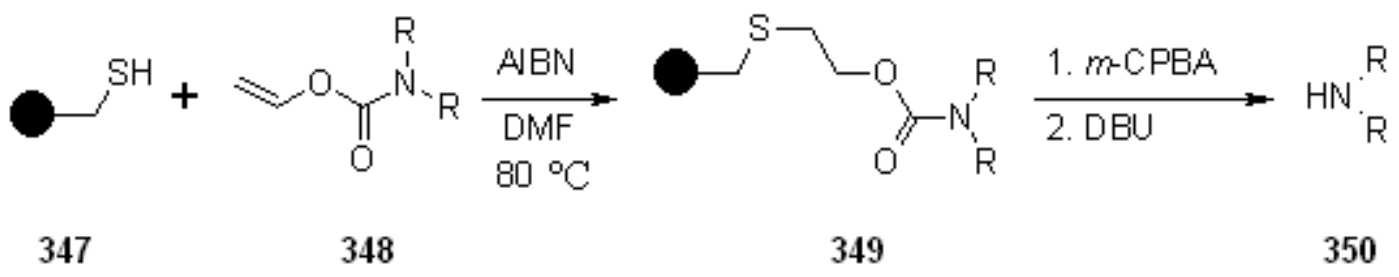

A possible problem in SP intermolecular radical reactions consists of the lower rate constants compared to the reactions run in solution, probably due to the heterogeneous nature of SP reactions. Zard's group [135] tried to address this problem by synthesizing a Wang-type soluble support. This support was then compared with the classical polystyrene Wang resin, via the radical addition of supported xanthates to olefins in solution and by the addition of immobilized olefins to free xanthates. Soluble support 357 was synthesized by xanthate polymerization and employed for intermolecular test radical addition. (Scheme 123)

Scheme 123. Zard's synthesis of soluble support for radical cyclization.

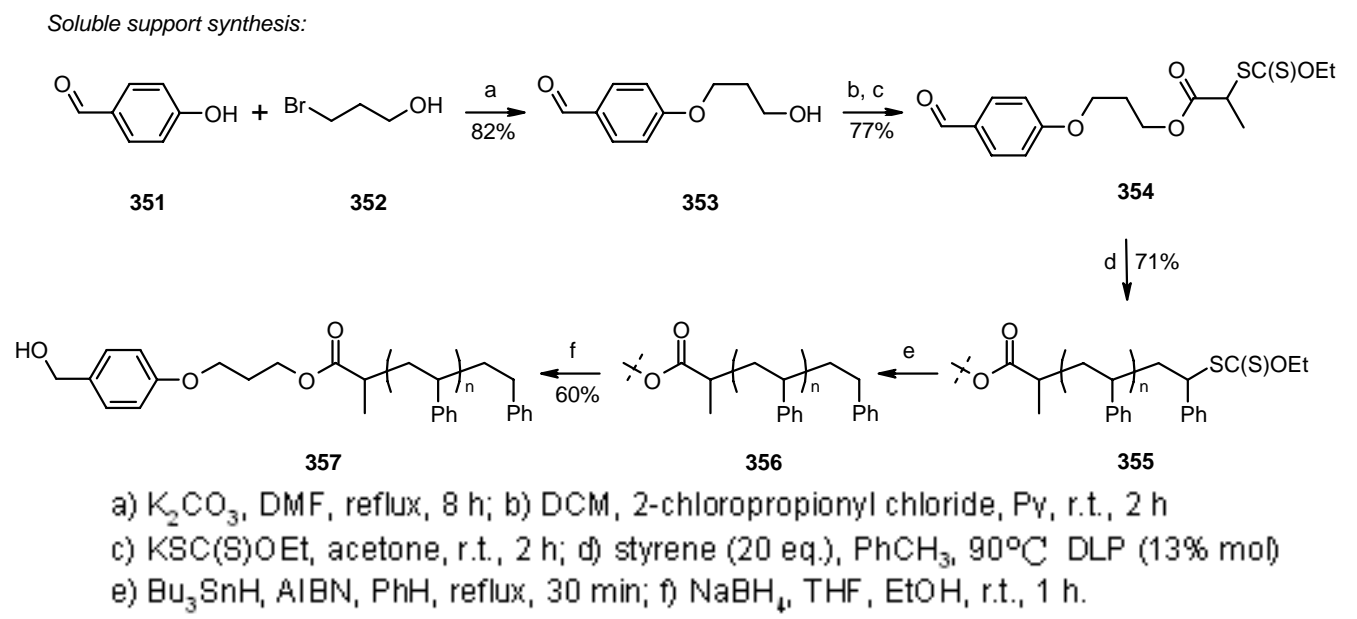


In Scheme 124 the supported xanthate was reacted with an excess olefin $\mathbf{3 6 0}$ under tin-free radical conditions. For both resins, the reaction was biased by the formation of by-products, compared to the one run without exploiting polymers. However, 359b (soluble support) gave cleaner reaction profile and higher yields (54\% vs. 26\%) compared to 359a (Wang). For Wang resin a large excess of olefin 360 and 30\% mol of lauroyl peroxide (DLP) needed to be used, while for the soluble support, a 3-fold excess of 360 and $40 \%$ DLP were needed to complete the reaction.

Scheme 124. Zard's use of supported xanthates.

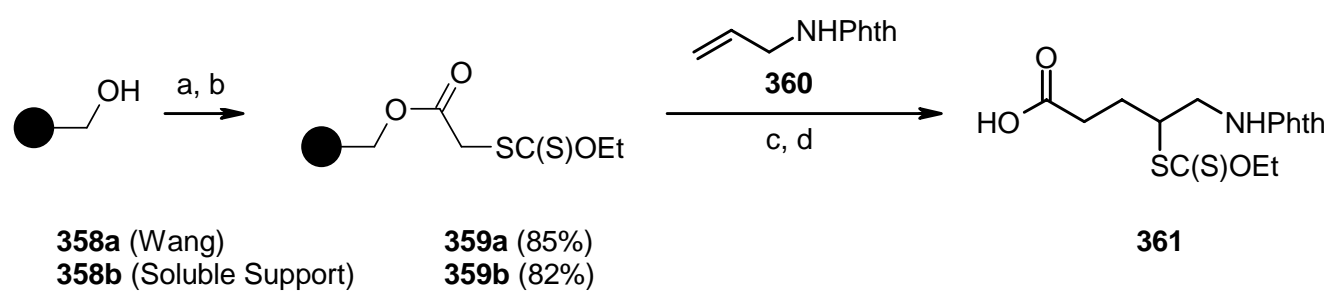

a) $\mathrm{ClCOCH}_{2} \mathrm{Cl}$ or $\mathrm{BrCOCH}_{2} \mathrm{Cl}$, Et $\mathrm{Cl}_{3} \mathrm{~N}$, r.t., $2 \mathrm{~h}$; b) $\mathrm{KSC}(\mathrm{S}) \mathrm{OEt}$, acetone, r.t., $2 \mathrm{~h}$

c) 1,2-DCE, reflux, DLP; d) $10 \%$ TFA-DCM, r.t., $30 \mathrm{~min}$.

When the reagents were inverted (Scheme 125), in both cases, the reaction did not go to completion and side-product $\mathbf{3 6 5}$ was found in similar proportion to the desired product. Despite this, the reaction was substantially cleaner compared to the one described in Scheme 124, even if it was still needed a consistent amount of DLP $(50 \% \mathrm{~mol})$ compared to the polymer-free reaction. Final yields were reasonable (52\% for Wang, 70\% for soluble support). The presence of side product 365 says that at certain xanthate concentrations, the intermolecular reaction is in competition with the intramolecular process, preventing the reaction to go forward in the desired direction. These results suggest that intermolecular radical processes are viable on SP and that the use of soluble support has more benefits compared to the heterogeneous classical SP synthesis.

Scheme 125. Zard's use of supported alkenes.

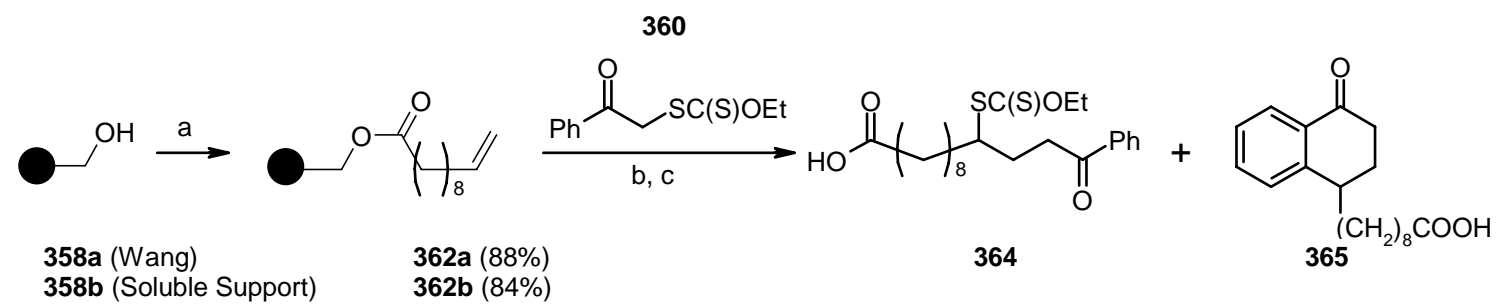

a) 10-undecenoyl chloride, $\mathrm{Et}_{3} \mathrm{~N}$, r.t., $2 \mathrm{~h}$;

c) 1,2-DCE, reflux, DLP; d) 10\% TFA-DCM, r.t., $30 \mathrm{~min}$.

Back and Zhai [136] performed a radical addition of PS-arylselenosulfonates to alkynes (Scheme 126).

Scheme 126. Back’s SP-radical addition of resin 366 to free alkynes.

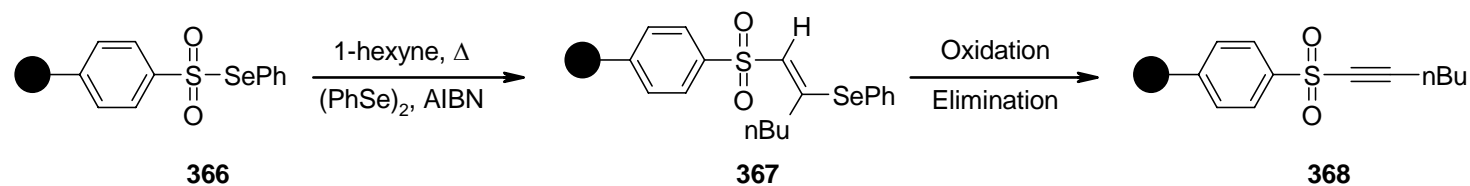


Scheme 127. Back's SP-radical addition of resin 369 to free alkynes.

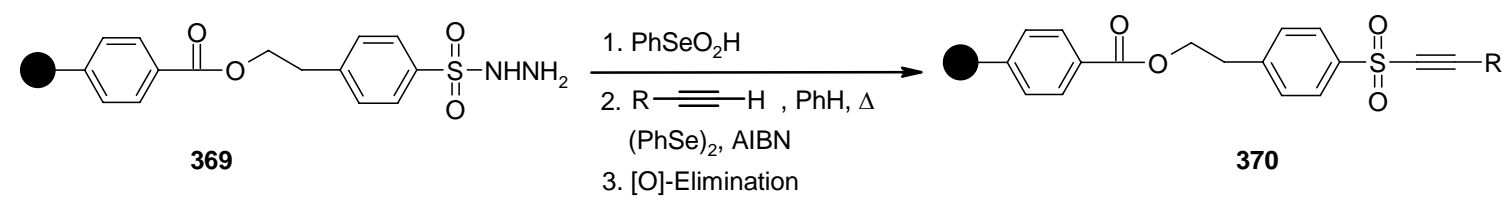

$\mathrm{R}=\mathrm{Ph}, n-\mathrm{Bu}, \mathrm{TMS}$

Acetylenic sulfones of the kind of $\mathbf{3 6 8}$ (or 370) are useful synthones for a variety of reactions (such as cycloadditions). Their first attempt to perform their synthesis on SP through radical addition to 1-hexyne is displayed in Scheme 127. Radical reaction and subsequent oxidation-elimination to restore the triple bond were effective, however, when $\mathbf{3 6 8}$ was further reacted under cyclization conditions, it did not yield any product. For this reason, it was added a spacer to the SP, therefore, resin $\mathbf{3 6 9}$ was prepared and reacted to yield products $\mathbf{3 7 0}$. Finally, $\mathbf{3 7 0}$ were successfully reacted under cyclization conditions, giving the desired products in good yields.

In the quest for new methodologies viable for the synthesis of small molecules on SP, Attardi and Taddei [137] presented a radical approach for the construction of unnatural amino acids and their small oligopeptides (Scheme 128).

Scheme 128. Taddei's SP-synthesis of unnatural AAs by radical approach.<smiles>CCOC(=O)CNCCCC(=O)On1ccccc1=S</smiles>

371

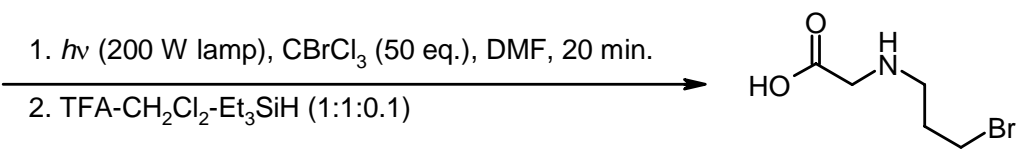

372

In the example reported above the Wang resin anchored Barton ester 371 was obtained by coupling of the correspondent acid with 1-hydroxy-2-pyridinethione. The photolabile product was submitted to irradiation to give radical fragmentation and loss of $\mathrm{CO}_{2}$, followed by trapping with $\mathrm{CBrCl}_{3}$. To minimize side-products it was necessary the use of DMF as solvent and 50 equivalents of the halogenated chemical reagent. After cleavage, a respectable $72 \%$ yield was obtained. The same process was applied to di- and tri-peptides, while the resulting alkylbromides were further reacted with nucleophiles to obtain the desired unnatural aminoacid side-chain.

\subsection{Other}

Troc (2,2,2-trichloroethoxycarbonyl) group is a widely used protecting group in organic, especially oligosaccharide, chemistry. Since its removal is often performed under heterogeneous conditions, it is not ideal for SP chemistry. Fukase [105] studied a methodology for Troc removal on a substrate anchored to SP, performed using a radical methodology (Scheme 129). The conditions used were tested in solution before extension of the methodology to SP and it was found that DMF was the best solvent (the reaction did not proceed in toluene), probably due to a concentration effect of the organotin derivative to the polymer support; and that the reactions proceeded slowly under conventional heating, therefore, microwave irradiation seemed to be the best choice. Since the amount 
of $\left(\mathrm{Bu}_{3} \mathrm{Sn}\right)_{2}$ was substoichiometric, an involvement of DMF in the catalytic cycle was also suggested. Beside the high yields obtained, the method was also effective because of the absence of dichloroethoxycarbonylated byproducts.

Scheme 129. Fukase's SP Troc radical deprotection.

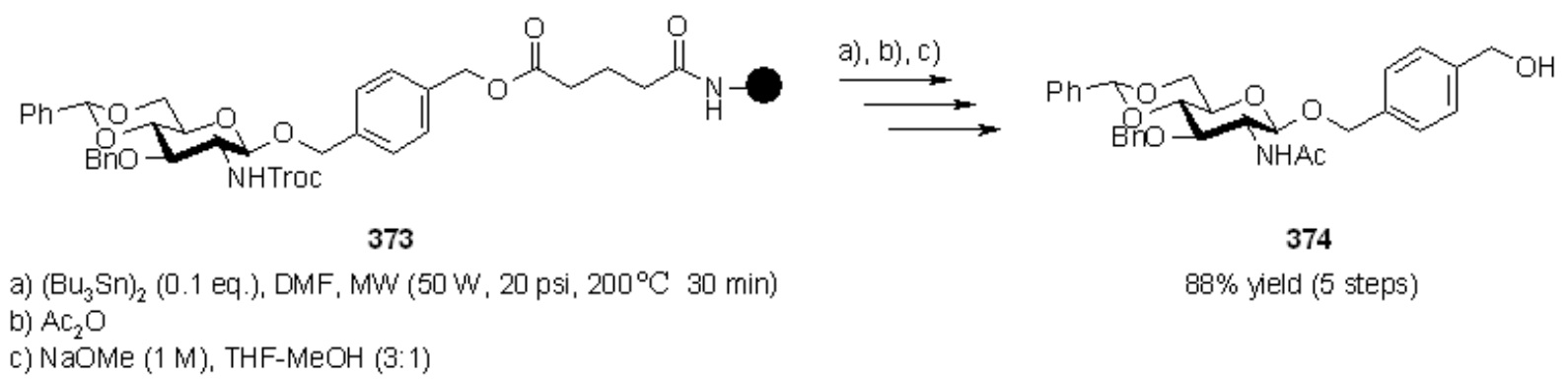

The couple 2-aminobenzoic acid (Abz) and 3-nitrotyrosine $\left(\operatorname{Tyr}\left(3-\mathrm{NO}_{2}\right)\right)$ is used as fluorophore-quencher combination in FRET (Fluorescence Resonance Energy Transfer) methodology for monitoring structural, functional, or aggregation changes in evaluated biomolecules. Since the couple can be easily incorporated in peptides, it would be of great advantage having a method for selective nitration of tyrosine incorporated on a peptide anchored to SP. Savinov and colleagues [138] did find this very mild, SP-friendly methodology for selective nitration of phenols. After a careful optimization of the reaction conditions and a mechanistic study, the reaction was tested on resin-bound substrates (Scheme 130).

Scheme 130. Savinov's nitration of phenols.

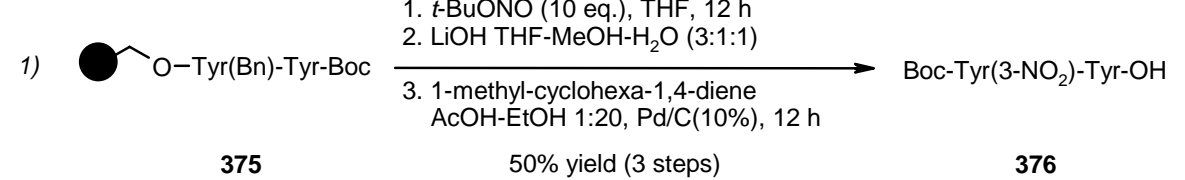

2) O-Asp(OBn)-Tyr-Gly-Gln-Phe-Ahx-Abz-Boc
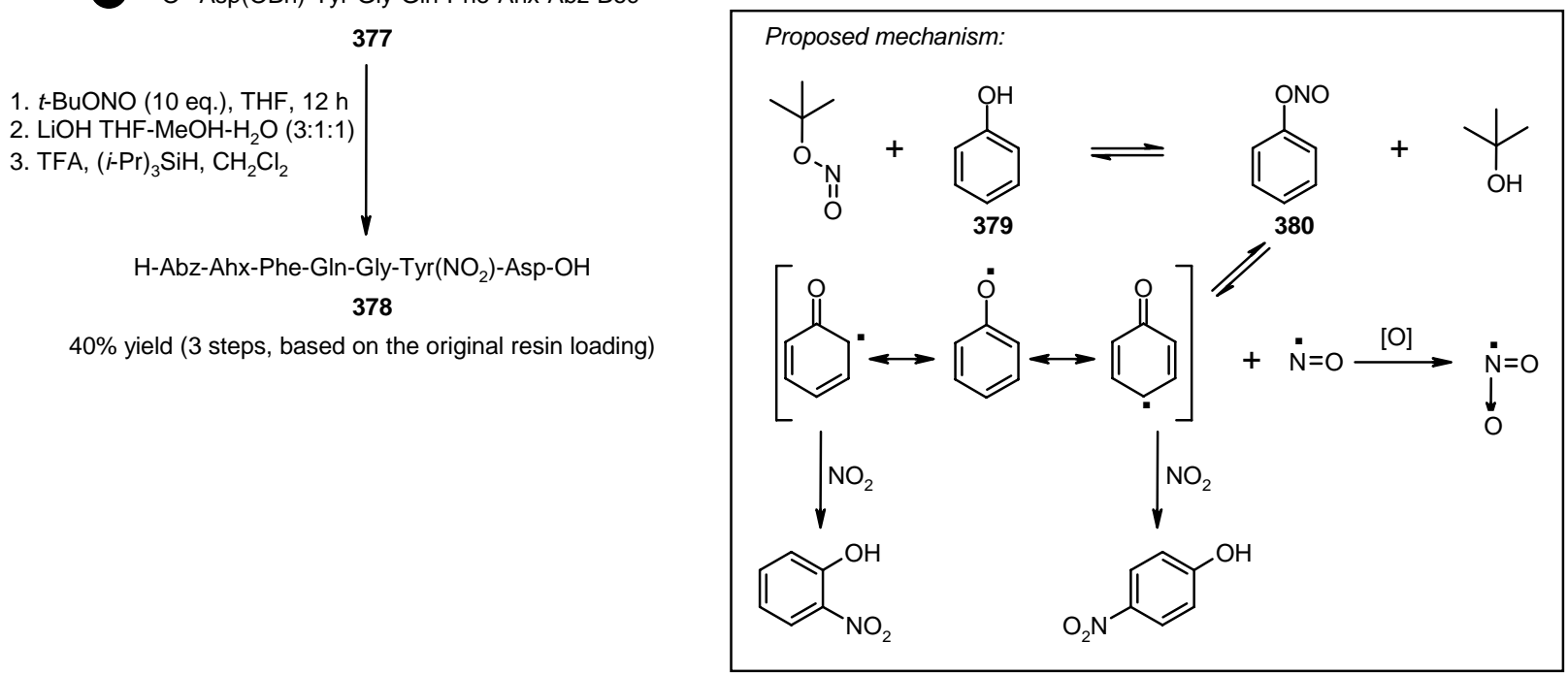
The right solvent for the reaction (THF) was first identified in solution, where some evidence of chemoselectivity was also found. Most importantly, the reaction worked only when a phenol hydroxyl group was present. Subsequently, the protocol was tested on dipeptide 375, confirming the need for a free phenolic hydroxyl group and, finally, on a peptide such as 376, giving the desired nitrotyrosine derivative $\mathbf{3 7 8}$ in good yield. The hypothesized mechanism was confirmed by the isolation of the $O$-nitroso derivative (such as 380) and the observation of the stabilized phenoxyl radical of 379, with its characteristic blue color. Decomposition of an $O$-nitroso derivative such as $\mathbf{3 8 0}$ led to nitric oxide, which underwent oxidation (probably from air oxygen) to nitrogen dioxide that subsequently coupled with the phenoxyl radical to give the nitration products. It is clear from the proposed mechanism, that the phenol hydroxyl group participates in the reaction, in fact, when anisole was subjected to the same experimental conditions, no nitrated products were observed.

\section{Conclusions}

Radical chemistry, despite having a long-standing tradition and deep relationships with polymers, has become one of the available means for solid-phase synthesis only in relatively recent years. Nevertheless, the wealth of the examples we have provided above witnesses the fruition of such a merging.

On the other hand, in the same period of time the concept of diversity-oriented synthesis (DOS) [12] has abruptly emerged in organic chemistry, with the goal of providing collections of small molecules. A number of tools are available to achieve such an end and in this context, as this review has illustrated, radical chemistry applied to SP represents an important and valid alternative.

\section{Acknowledgements}

The authors would like to gratefully acknowledge Paolo Trifirò for his helpful assistance.

\section{References and Notes}

1. Gomberg, M. An Instance of Trivalent Carbon: Triphenylmethyl. J. Am. Chem. Soc. 1900, 22, 757-771.

2. Geng, Y.; Discher, D.E.; Justynska, J.; Schlaad, H. Grafting Short Peptides onto Polybutadieneblock-poly(ethylene oxide): A Platform for Self-Assembling Hybrid Amphiphiles. Angew. Chem. Int. Ed. Engl. 2006, 45, 7578-7581.

3. Siegenthaler, K.O; Schäfer, A.; Studer, A. Chemical Surface Modification via Radical C-C Bond-Forming Reactions. J. Am. Chem. Soc. 2007, 129, 5826-5827.

4. Hensarling, R.M.; Doughty, V.A.; Chan, J.W.; Patton, D.L. Clicking Polymer Brushes with Thiol-yne Chemistry: Indoors and Out. J. Am. Chem. Soc. 2009, 131, 14673-14675.

5. Walling, C. Some properties of radical reactions important in synthesis. Tetrahedron 1985, 41, 3887-3900.

6. Newcomb, M. Competition Methods and Scales for Alkyl Radical Reaction Kinetics. Tetrahedron 1993, 49, 1151-1176. 
7. Gansäuer, A.; McGhee, A.; Procter, D. Radical Chemistry on Solid Support, In Radicals in Synthesis II, Springer: Berlin/Heidelberg, Germany, 2006; Vol. 264, pp. 93-134.

8. Curran, D.P.; Yang, F.; Cheong, J.H. Relative Rates and Approximate Rate Constants for Interand Intramolecular Hydrogen Transfer Reactions of Polymer-Bound Radicals. J. Am. Chem. Soc. 2002, 124, 14993-15000.

9. Lloyd-Williams, P.; Albericio, F.; Giralt, E. Convergent solid-phase peptide synthesis. Tetrahedron 1993, 49, 11065-11133.

10. Bochet, C.G. Photolabile protecting groups and linkers. J. Chem. Soc. Perkin Trans. 1 2002, 125-142.

11. Reitz, A.B. Recent advances in traceless linkers. Curr. Opin. Drug Discov. Dev. 1999, 2, 358-364.

12. Schreiber, S.L. Target-Oriented and Diversity-Oriented Organic Synthesis in Drug Discovery. Science 2000, 287, 1964-1969.

13. Scott, P.J.H.; Steel, P.G. Diversity Linker Units for Solid-Phase Organic Synthesis. Eur. J. Org. Chem. 2006, 2006, 2251-2268.

14. Jung, K.W.; Zhao, X.Y.; Janda, K.D. A linker that allows efficient formation of aliphatic C-H bonds on polymeric supports. Tetrahedron Lett. 1996, 37, 6491-6494.

15. Barluenga, S.; Dakas, P.Y.; Ferandin, Y.; Meijer, L.; Winssinger, N. Modular Asymmetric Synthesis of Aigialomycin D, a Kinase-Inhibitory Scaffold. Angew. Chem. Int. Ed. Engl. 2006, 45, 3951-3954.

16. Parsons, A.F.; Pettifer, R.M. A radical approach to N-desulfonylation. Tetrahedron Lett. 1996, 37, 1667-1670.

17. Luo, J.; Huang, W. A new strategy for solid phase synthesis of a secondary amide library using sulfonamide linker via radical traceless cleavage. Mol. Divers. 2003, 6, 33-41.

18. D'Herde, J.N.P.; J. de Clercq, P. Carbon-carbon bond formation on solid support. Application of the classical Julia-Lythgoe olefination. Tetrahedron Lett. 2003, 44, 6657-6659.

19. Allin, S.M.; Bowman, W.R.; Karim, R.; Rahman, S.S. Aromatic homolytic substitution using solid phase synthesis. Tetrahedron 2006, 62, 4306-4316.

20. Roberts, B.P. Polarity-reversal catalysis of hydrogen-atom abstraction reactions: concepts and applications in organic chemistry. Chem. Soc. Rev. 1999, 28, 25-35.

21. McAllister, L.A.; Brand, S.; de Gentile, R.; Procter, D.J. The first Pummerer cyclisations on solid phase. Convenient construction of oxindoles enabled by a sulfur-link to resin. Chem. Commun. (Camb.) 2003, 2380-2381.

22. Turner, K.L.; Baker, T.M.; Islam, S.; Procter, D.J.; Stefaniak, M. Solid-Phase Approach to Tetrahydroquinolones Using a Sulfur Linker Cleaved by SmI 2 . Org. Lett. 2006, 8, 329-332.

23. McAllister, L.A.; Turner, K.L.; Brand, S.; Stefaniak, M.; Procter, D.J. Solid Phase Approaches to N-Heterocycles Using a Sulfur Linker Cleaved by $\mathrm{SmI}_{2}$. J. Org. Chem. 2006, 71, 6497-6507.

24. McAllister, L.A.; McCormick, R.A.; Procter, D.J. Sulfide- and selenide-based linkers in phase tag-assisted synthesis. Tetrahedron 2005, 61, 11527-11576.

25. Ruhland, T.; Andersen, K.; Pedersen, H. Selenium-Linking Strategy for Traceless Solid-Phase Synthesis: Direct Loading, Aliphatic C-H Bond Formation upon Cleavage and Reaction Monitoring by Gradient MAS NMR Spectroscopy. J. Org. Chem. 1998, 63, 9204-9211. 
26. Ruhland, T.; Torang, J.; Pedersen, H.; Madsen, J.C.; Bang, K.S. Traceless Solid Phase Synthesis with Polystyrene-Bound Tellurium and in Comparison with Polystyrene-Bound Selenium. Synthesis 2004, 2323-2328.

27. Ruhland, T.; Torang, J.; Pedersen, H.; Madsen, J.C.; Bang, K.S. Green Traceless Cleavage from Resin-Bound Selenium and Tellurium and Analysis by $2 \mathrm{D}{ }^{29} \mathrm{Si} /{ }^{1} \mathrm{H}$ HR-MAS NMR Spectroscopy. Synthesis 2005, 1635-1640.

28. Nicolaou, K.C.; Pastor, J.; Barluenga, S.; Winssinger, N. Polymer-supported selenium reagents for organic synthesis. Chem. Commun. (Camb.) 1998, 1947-1948.

29. Nicolaou, K.C.; Mitchell, H.J.; Fylaktakidou, K.C.; Suzuki, H.; Rodríguez, R.M. 1,2-Seleno Migrations in Carbohydrate Chemistry: Solution and Solid-Phase Synthesis of 2-Deoxy Glycosides, Orthoesters, and Allyl Orthoesters. Angew. Chem. Int. Ed. Engl. 2000, 39, 1089-1093.

30. Nicolaou, K.C.; Fylaktakidou, K.C.; Mitchell, H.J.; van Delft, F.L.; Rodríguez, R.M.; Conley, S.R.; Jin, Z. Total Synthesis of Everninomicin 13,384-1-Part 4: Explorations of Methodology; Stereocontrolled Synthesis of 1,1'-Disaccharides, 1,2-Seleno Migrations in Carbohydrates, and Solution- and Solid-Phase Synthesis of 2-Deoxy Glycosides and Orthoesters. Chem. Eur. J. 2000, 6, 3166-3185.

31. Nicolaou, K.C.; Roecker, A.J.; Pfefferkorn, J.A.; Cao, G.Q. A Novel Strategy for the Solid-Phase Synthesis of Substituted Indolines. J. Am. Chem. Soc. 2000, 122, 2966-2967.

32. Nicolaou, K.C.; Roecker, A.J.; Hughes, R.; van Summeren, R.; Pfefferkorn, J.A.; Winssinger, N. Novel strategies for the solid phase synthesis of substituted indolines and indoles. Bioorg. Med. Chem. 2003, 11, 465-476.

33. Nicolaou, K.C.; Pfefferkorn, J.A.; Cao, G.Q.; Kim, S.; Kessabi, J. A Facile Method for the Solution and Solid-Phase Synthesis of Substituted [3.3.1] Bicycles. Org. Lett. 1999, 1, 807-810.

34. Berlin, S.; Ericsson, C.; Engman, L. Radical Carbonylation/Reductive Cyclization for the Construction of Tetrahydrofuran-3-ones and Pyrrolidin-3-ones. J. Org. Chem. 2003, 68, 8386-8396.

35. Fujita, K.I.; Watanabe, K.; Oishi, A.; Ikeda, Y.; Taguchi, Y. Preparation of Polymer-Supported Selenocyanates and their Application to Solid-Phase Oxyselenenylation-Deselenylation. Synlett 1999, 1999, 1760-1762.

36. Qian, H.; Huang, X. Polystyrene-Supported Selenosulfonates: Efficient Reagents for Regio- and Stereocontrolled Synthesis of Vinyl Sulfones. Synlett 2001, 1913-1916.

37. Qian, H.; Huang, X. Polystyrene-supported selenosulfonates: efficient reagents for the synthesis of acetylenic sulfones. Tetrahedron Lett. 2002, 43, 1059-1061.

38. Qian, H.; Huang, X. Solid-Phase Synthesis of alpha-Keto Sulfones. Synthesis 2006, 1934-1936.

39. Qian, H.; Huang, X. Radical Cyclization of 1,6-Diene Using Polystyrene-Supported Selenosulfones. J. Comb. Chem. 2003, 5, 569-576.

40. Myers, R.M.; Langston, S.P.; Conway, S.P.; Abell, C. Reductive Cleavage of N-O Bonds Using Samarium(II) Iodide in a Traceless Release Strategy for Solid-Phase Synthesis. Org. Lett. 2000, 2, 1349-1352. 
41. Gustafsson, M.; Olsson, R.; Andersson, C.M. General combinatorial synthesis of tertiary amines on solid support. A novel conditional release strategy based on traceless linking at nitrogen. Tetrahedron Lett. 2001, 42, 133-136.

42. Meloni, M.M.; Taddei, M. Solid-Phase Synthesis of beta-Lactams via the Miller Hydroxamate Approach. Org. Lett. 2001, 3, 337-340.

43. Kerrigan, N.J.; Hutchison, P.C.; Heightman, T.D.; Procter, D.J. Application of an ephedrine chiral linker in a solid-phase, 'asymmetric catch-release' approach to gamma-butyrolactones. Chem. Commun. (Camb.) 2003, 21, 1402-1403.

44. Kerrigan, N.J.; Hutchison, P.C.; Heightman, T.D.; Procter, D.J. Development of a solid-phase 'asymmetric resin-capture-release' process: application of an ephedrine chiral resin in an approach to gamma-butyrolactones. Org. Biomol. Chem. 2004, 2, 2476-2482.

45. Zhang, Y.; Wang, Y.; Dai, W.M. Efficient Remote Axial-to-Central Chirality Transfer in Enantioselective $\mathrm{SmI}_{2}$-Mediated Reductive Coupling of Aldehydes with Crotonates of Atropisomeric 1-Naphthamides. J. Org. Chem. 2006, 71, 2445-2455.

46. McKerlie, F.; Procter, D.J.; Wynne, G. Reduction of alpha-aryloxy carbonyl compounds with samarium(II) iodide. A new traceless linker for the solid phase synthesis of carbonyl compounds. Chem. Commun. (Camb.) 2002, 21, 584-585.

47. McKerlie, F.; Rudkin, I.M.; Wynne, G.; Procter, D.J. Evaluation of a new linker system cleaved using samarium(II) iodide. Application in the solid phase synthesis of carbonyl compounds. Org. Biomol. Chem. 2005, 3, 2805-2816.

48. Liu, H.; Wan, S.; Floreancig, P.E. Oxidative Cyclorelease from Soluble Polymeric Supports. J. Org. Chem. 2005, 70, 3814-3818.

49. Floreancig, P.E. Development and Applications of Electron-Transfer-Initiated Cyclization Reactions. Synlett 2007, 38, 191-203.

50. Whitehead, D.M.; Helliwell, P.A.; McKeown, S.C.; Routledge, A. Synthesis, chemical stability and application of a thiohydroxamic acid linker (THA) for solid-phase organic synthesis. React. Funct. Polym. 2009, 69, 884-890.

51. Horton, J.R.; Stamp, L.M.; Routledge, A. A photolabile 'traceless' linker for solid-phase organic synthesis. Tetrahedron Lett. 2000, 41, 9181-9184.

52. Whitehead, D.M.; Jackson, T.; McKeown, S.C.; Wilson, K.; Routledge, A. Application of X-ray Photoelectron Spectroscopy in Determining the Structure of Solid-Phase Bound Substrates. $J$. Comb. Chem. 2002, 4, 255-257.

53. Gerigk, U.; Gerlach, M.; Neumann, W.P.; Vieler, R.; Weintritt, V. Polymer-Supported Organotin Hydrides as Immobilized Reagents for Free Radical Synthesis. Synthesis 1990, 1990, 448-452.

54. Gerlach, M.; Joerdens, F.; Kuhn, H.; Neumann, W.P.; Peterseim, M. A polymer-supported organotin hydride and its multipurpose application in radical organic synthesis. J. Org. Chem. 1991, 56, 5971-5972.

55. Barton, D.H.R.; McCombie, S.W. A new method for the deoxygenation of secondary alcohols. $J$. Chem. Soc., Perkin Trans. 1 1975, 1574-1585.

56. Neumann, W.P.; Peterseim, M. Elegant Improvement of the Deoxygenation of Alcohols Using a Polystyrene-Supported Organotin Hydride. Synlett 1992, 1992, 801-802. 
57. Ruel, G.; Ngo, K.T.; Dumartin, G.; Delmond, B.; Pereyre, M. Un nouvel hydrure organostannique greffé sur support insoluble. J. Organomet. Chem. 1993, 444, C18-C20.

58. Dumartin, G.; Ruel, G.; Kharboutli, J.; Delmond, B.; Connil, M.F.; Jousseaume, B.; Pereyre, M. Straightforward Synthesis and Reactivity of Polymer-supported Organotin Hydrides. Synlett 1994, 1994, 952-954.

59. Dumartin, G.; Pourcel, M.; Delmond, B.; Donard, O.; Pereyre, M. In situ generated, polymersupported organotin hydrides as clean reducing agents. Tetrahedron Lett. 1998, 39, 4663-4666.

60. Chemin, A.; Deleuze, H.; Maillard, B. Preparation and reactivity of a macroporous polymersupported organotin hydride catalyst. Eur. Polym. J. 1998, 34, 1395-1404.

61. Boussaguet, P.; Delmond, B.; Dumartin, G.; Pereyre, M. Catalytic and supported BartonMcCombie deoxygenation of secondary alcohols: a clean reaction. Tetrahedron Lett. 2000, 41, 3377-3380.

62. Lopez, R.M.; Hays, D.S.; Fu, G.C. Bu $\mathrm{Bu}_{3} \mathrm{SnH}-\mathrm{Catalyzed} \mathrm{Barton-McCombie} \mathrm{Deoxygenation} \mathrm{of}$ Alcohols. J. Am. Chem. Soc. 1997, 119, 6949-6950.

63. Harendza, M.; Lessmann, K.; Neumann, W.P. A Polymer-Supported Distannane as Photochemical, Regenerable Source of Stannyl Radicals for Organic Synthesis. Synlett 1993, 1993, 283-285.

64. Junggebauer, J.; Neumann, W.P. An improved synthesis of a polymer-supported distannane and its application to radical formation. Tetrahedron 1997, 53, 1301-1310.

65. Enholm, E.J.; Gallagher, M.E.; Moran, K.M.; Lombardi, J.S.; Schulte, J.P. An Allylstannane Reagent on Non-Cross-Linked Polystyrene Support. Org. Lett. 1999, 1, 689-691.

66. Enholm, E.J.; Schulte, J.P. Convenient Catalytic Free Radical Reductions of Alkyl Halides Using an Organotin Reagent on Non-Cross-Linked Polystyrene Support. Org. Lett. 1999, 1, 1275-1277.

67. Corey, E.J.; Suggs, J.W. Method for catalytic dehalogenations via trialkyltin hydrides. J. Org. Chem. 1975, 40, 2554-2555.

68. Ueno, Y.; Chino, K.; Watanabe, M.; Moriya, O.; Okawara, M. Homolytic carbocyclization by use of a heterogeneous supported organotin catalyst. A new synthetic route to 2-alkoxytetrahydrofurans and gamma-butyrolactones. J. Am. Chem. Soc. 1982, 104, 5564-5566.

69. Mochida, K.; Sugimoto, H.; Yasuo, Y. First polymer-supported organogermanium hydrides and their reduction of organic halides. Polyhedron 1997, 16, 1767-1770.

70. Bowman, W.R.; Krintel, S.L.; Schilling, M.B. New Solid Phase Triorganogermanium Hydrides for Radical Synthesis. Synlett 2004, 2004, 1215-1218.

71. Patro, B.; Merrett, M.; Murphy, J.A.; Sherrington, D.C.; Morrison, M.G.J.T. Radical-polar crossover reactions with polymer-supported tetrathiafulvalene. Tetrahedron Lett. 1999, 40, 7857-7860.

72. Baguley, P.A.; Walton, J.C. Flight from the Tyranny of Tin: The Quest for Practical Radical Sources Free from Metal Encumbrances. Angew. Chem. Int. Ed. Engl. 1998, 37, 3072-3082.

73. Fletcher, R.; Kizil, M.; Lampard, C.; Murphy, J.A.; Roome, S.J. Tetrathiafulvalene-mediated stereoselective synthesis of the tetracyclic core of Aspidosperma alkaloids. J. Chem. Soc., Perkin Trans. 1 1998, 2341-2352.

74. Callaghan, O.; Lampard, C.; Kennedy, A.R.; Murphy, J.A. A novel total synthesis of (+/-)aspidospermidine. J. Chem. Soc., Perkin Trans. 1 1999, 995-1002. 
75. de Luca, L.; Giacomelli, G.; Porcu, G.; Taddei, M. A New Supported Reagent for the Photochemical Generation of Radicals in Solution. Org. Lett. 2001, 3, 855-857.

76. Barton, D.H.R.; Crich, D.; Motherwell, W.B. The invention of new radical chain reactions. Part VIII. Radical chemistry of thiohydroxamic esters; A new method for the generation of carbon radicals from carboxylic acids. Tetrahedron 1985, 41, 3901-3924.

77. Clark, A.J.; Filik, R.P.; Haddleton, D.M.; Radigue, A.; Sanders, C.J.; Thomas, G.H.; Smith, M.E. Solid-Supported Catalysts for Atom-Transfer Radical Cyclization of 2-Haloacetamides. J. Org. Chem. 1999, 64, 8954-8957.

78. Clark, A.J.; Geden, J.V.; Thom, S. Solid-Supported Copper Catalysts for Atom-Transfer Radical Cyclizations: Assessment of Support Type and Ligand Structure on Catalyst Performance in the Synthesis of Nitrogen Heterocycles. J. Org. Chem. 2006, 71, 1471-1479.

79. Baldwin, J.E. Rules for ring closure. J. Chem. Soc. Chem. Commun. 1976, 734-736.

80. Baldwin, J.E.; Cutting, J.; Dupont, W.; Kruse, L.; Silberman, L.; Thomas, R.C. 5-Endo-trigonal reactions: a disfavoured ring closure. J. Chem. Soc. Chem. Commun. 1976, 736-738.

81. Clark, A.J.; Dell, C.P.; Ellard, J.M.; Hunt, N.A.; McDonagh, J.P. Efficient room temperature copper(I) mediated 5-endo radical cyclisations. Tetrahedron Lett. 1999, 40, 8619-8623.

82. Motoyama, Y.; Kamo, K.; Yuasa, A.; Nagashima, H. Catalytic atom-transfer radical cyclization by copper/bipyridine species encapsulated in polysiloxane. Chem. Commun. (Camb.) 2010, 46, 2256-2258.

83. Teduka, T.; Togo, H. I2-Mediated Photochemical Preparation of 2-Substituted 1,3-Dioxolanes and Tetrahydrofurans from Alcohols with Polymer-Supported Hypervalent Iodine Reagent, PSDIB. Synlett 2005, 2005, 923-926.

84. Kashiwagi, Y.; Ikezoe, H.; Ono, T. Oxidation of Alcohols with Nitroxyl Radical Resins under Two-Phase Conditions. Synlett 2006, 2006, 69-72.

85. Sibi, M.P.; Chandramouli, S.V. Intermolecular free radical reactions on solid support. Allylation of esters. Tetrahedron Lett. 1997, 38, 8929-8932.

86. Enholm, E.J.; Gallagher, M.E.; Jiang, S.; Batson, W.A. Free Radical Allyl Transfers Utilizing Soluble Non-Cross-Linked Polystyrene and Carbohydrate Scaffold Supports. Org. Lett. 2000, 2, 3355-3357.

87. Enholm, E.J.; Gallagher, M.E. Free Radical Reactions on Soluble Supports from Ring-Opening Metathesis. Org. Lett. 2001, 3, 3397-3399.

88. Baytas, S.N.; Wang, Q.; Karst, N.A.; Dordick, J.S.; Linhardt, R.J. Solid-Phase Chemoenzymatic Synthesis of C-Sialosides. J. Org. Chem. 2004, 69, 6900-6903.

89. Zhu, X.; Ganesan, A. Intermolecular Conjugate Addition of Alkyl Radicals on Solid Phase. J. Comb. Chem. 1999, 1, 157-162.

90. Kumar, H.M.S.; Chakravarthy, P.P.; Rao, M.S.; Reddy, P.S.R.; Yadav, J.S. Free radical addition of haloalkanes to polymer bound olefins and its application to the solid-phase synthesis of pyrethroids. Tetrahedron Lett. 2002, 43, 7817-7819.

91. Yim, A.M.; Vidal, Y.; Viallefont, P.; Martinez, J. Solid-phase synthesis of alpha-amino acids by radical addition to adehydroalanine derivative. Tetrahedron Lett. 1999, 40, 4535-4538. 
92. Caddick, S.; Hamza, D.; Wadman, S.N.; Wilden, J.D. Solid-Phase Intermolecular Radical Reactions 2: Synthesis of C-Glycopeptide Mimetics via a Novel Acrylate Acceptor. Org. Lett. 2002, 4, 1775-1777.

93. Miyabe, H.; Fujishima, Y.; Naito, T. Novel Synthesis of alpha-Amino Acid Derivatives through Triethylborane-Induced Solid-Phase Radical Reactions. J. Org. Chem. 1999, 64, 2174-2175.

94. Miyabe, H.; Nishimura, A.; Fujishima, Y.; Naito, T. Carbon-carbon bond construction on solid support: triethylborane-induced radical reactions of oxime ethers. Tetrahedron 2003, 59, 1901-1907.

95. Miyabe, H.; Ueda, M.; Naito, T. Carbon-Carbon Bond Construction Based on Radical Addition to C=N Bond. Synlett 2004, 2004, 1140-1157.

96. Miyabe, H. Development of Solid-Phase Radical Reactions Using Oxime Ethers as a Radical Acceptor. Yakugaku Zasshi 2000, 120, 667-676.

97. Miyabe, H. Development of Carbon Radical Addition to Imine Derivatives. Yakugaku Zasshi 2003, 123, 285-294.

98. Miyabe, H.; Konishi, C.; Naito, T. Stereocontrol in Solid-Phase Radical Reactions: Radical Addition to Oxime Ether Anchored to Polymer Support. Org. Lett. 2000, 2, 1443-1445.

99. Miyabe, H.; Konishi, C.; Naito, T. Diastereoselective Solid-Phase Radical Addition to Oxime Ether Anchored to Polymer Support. Chem. Pharm. Bull. 2003, 51, 540-544.

100. Jeon, G.H.; Yoon, J.Y.; Kim, S.; Kim, S.S. Radical Reaction of Phenylsulfonyl Oxime Ethers on Solid Support: Application to the Synthesis of alpha-Amino Esters. Synlett 2000, 2000, 128-130.

101. Miyabe, H.; Tanaka, H.; Naito, T. Triethylborane-induced solid-phase radical cyclization of oxime ethers. Tetrahedron Lett. 1999, 40, 8387-8390.

102. Miyabe, H.; Fujii, K.; Tanaka, H.; Naito, T. Solid-phase tandem radical addition-cyclisation reaction of oxime ethers. Chem. Commun. (Camb.) 2001, 831-832.

103. Miyabe, H.; Tanaka, H.; Naito, T. Solid-Phase Tandem Radical Addition-Cyclization Reaction: Triethylborane-Induced Reaction of Oxime Ethers Anchored to Polymer Support. Chem. Pharm. Bull. 2004, 52, 842-847.

104. Akamatsu, H.; Fukase, K.; Kusumoto, S. Solid-Phase Synthesis of Indol-2-ones by MicrowaveAssisted Radical Cyclization. Synlett 2004, 2004, 1049-1053.

105. Tokimoto, H.; Fukase, K. New deprotection method of the 2,2,2-trichloroethoxycarbonyl (Troc) group with $\left(\mathrm{Bu}_{3} \mathrm{Sn}\right)_{2}$. Tetrahedron Lett. 2005, 46, 6831-6832.

106. Allin, S.M.; Bowman, W.R.; Elsegood, M.R.J.; McKee, V.; Karim, R.; Rahman, S.S. Synthetic applications of aryl radical building blocks for cyclisation onto azoles. Tetrahedron 2005, 61, 2689-2696.

107. Berteina, S.; de Mesmaeker, A.; Wendeborn, S. Functionalization via Radical Cyclization of Cyclohexenediol Derivatives Bound to Polystyrene. Synlett 1999, 1999, 1121-1123.

108. Du, X.; Armstrong, R.W. Synthesis of Benzofuran Derivatives on Solid Support via $\mathrm{SmI}_{2}$-Mediated Radical Cyclization. J. Org. Chem. 1997, 62, 5678-5679.

109. Du, X.; Armstrong, R.W. $\mathrm{SmI}_{2}$-mediated sequential radical cyclization/anionic capture of aryl iodides on solid support. Tetrahedron Lett. 1998, 39, 2281-2284.

110. Berteina, S.; Mesmaeker, A.D. Application of radical chemistry to solid support synthesis. Tetrahedron Lett. 1998, 39, 5759-5762. 
111. Berteina, S.; Wendeborn, S.; de Mesmaeker, A. Radical and Palladium-Mediated Cyclization of Ortho-Iodo Benzyl Enamines: Application to Solid Phase Synthesis. Synlett 1998, 1998, 1231-1233.

112. Chevet, C.; Jackson, T.; Santry, B.; Routledge, A. A Cleaner Approach to Solid-Supported Radical Chemistry: Application of Hypophosphite Salts to Intramolecular C-C Bond Formation on the Solid-Phase. Synlett 2005, 2005, 477-480.

113. Helliwell, P.A.; Bailey, V.A.; Chevet, C.; Clarke, D.B.; Lloyd, A.; Macarthur, R.; Routledge, A. A mass spectrometric investigation into microenvironmental effects in solid-supported radical chemistry. React. Funct. Polym. 2010, 70, 110-115.

114. Routledge, A.; Abell, C.; Balasubramanian, S. An Investigation into Solid-Phase Radical Chemistry-Synthesis of Furan Rings. Synlett 1997, 1997, 61-62.

115. Jia, G.; Iida, H.; Lown, J.W. Solid-Phase Synthesis of 1-Chloromethyl-1,2-dihydro-3Hbenz[e]indole (seco-CBI) and a Polyamide Conjugate. Synlett 2000, 2000, 603-606.

116. Watanabe, Y.; Ishikawa, S.; Takao, G.; Toru, T. Radical cyclization on solid support: Synthesis of gamma-butyrolactones. Tetrahedron Lett. 1999, 40, 3411-3414.

117. Enholm, E.J.; Cottone, J.S. Highly Diastereoselective Radical Cyclizations on Soluble Ring Opening Metathesis Supports. Org. Lett. 2001, 3, 3959-3962.

118. Renaud, P.; Gerster, M. Use of Lewis Acids in Free Radical Reactions. Angew. Chem. Int. Ed. Engl. 1998, 37, 2562-2579.

119. Murakata, M.; Jono, T.; Mizuno, Y.; Hoshino, O. Construction of Chiral Quaternary Carbon Centers by Catalytic Enantioselective Radical-Mediated Allylation of alpha-Iodolactones Using Allyltributyltin in the Presence of a Chiral Lewis Acid. J. Am. Chem. Soc. 1997, 119, 11713-11714.

120. Porter, N.A.; Giese, B.; Curran, D.P. Acyclic stereochemical control in free-radical reactions. Acc. Chem. Res. 1991, 24, 296-304.

121. Nishida, M.; Hayashi, H.; Yamaura, Y.; Yanaginuma, E.; Yonemitsu, O.; Nishida, A.; Kawahara, N. A simple preparation of (R)-(2-cyclopentenyl)acetic acid and (R)-(2-cyclohexenyl)acetic acid using beta-diastereoselective radical cyclization in the presence of Lewis acid. Tetrahedron Lett. 1995, 36, 269-272.

122. Nishida, M.; Ueyama, E.; Hayashi, H.; Ohtake, Y.; Yamaura, Y.; Yanaginuma, E.; Yonemitsu, O.; Nishida, A.; Kawahara, N. Lewis Acid-Promoted Diastereoselective Radical Cyclization Using Chiral alpha,beta-Unsaturated Esters. J. Am. Chem. Soc. 1994, 116, 6455-6456.

123. Badone, D.; Bernassau, J.M.; Cardamone, R.; Guzzi, U. Diastereoselective Chelation-Controlled Radical Cyclization of Chiral Oxazolidinone-Derived 2-Alkenamides and Modeling of the Transition State. Angew. Chem. Int. Ed. Engl. 1996, 35, 535-538.

124. Lamberto, M.; Corbett, D.F.; Kilburn, J.D. Thiol-mediated free radical cyclisations of isocyanides on solid support. Tetrahedron Lett. 2004, 45, 8541-8543.

125. Harrowven, D.C.; May, P.J.; Bradley, M. Sulfur-mediated radical cyclisation reactions on solid support. Tetrahedron Lett. 2003, 44, 503-506.

126. Beckwith, A.L.J.; Schiesser, C.H. Regio- and stereo-selectivity of alkenyl radical ring closure: A theoretical study. Tetrahedron 1985, 41, 3925-3941. 
127. Spellmeyer, D.C.; Houk, K.N. Force-field model for intramolecular radical additions. J. Org. Chem. 1987, 52, 959-974.

128. Nicolaou, K.C.; Baran, P.S.; Zhong, Y.L.; Choi, H.S.; Yoon, W.H.; He, Y.; Fong, K.C. Total Synthesis of the CP Molecules CP-263,114 and CP-225,917 - Part 1: Synthesis of Key Intermediates and Intelligence Gathering. Angew. Chem. Int. Ed. Engl. 1999, 38, 1669-1675.

129. Nicolaou, K.C.; Baran, P.S.; Zhong, Y.L.; Fong, K.C.; He, Y.; Yoon, W.H.; Choi, H.S. Total Synthesis of the CP Molecules CP-225,917 and CP-263,114 - Part 2: Evolution of the Final Strategy. Angew. Chem. Int. Ed. Engl. 1999, 38, 1676-1678.

130. Nicolaou, K.C.; Zhong, Y.L.; Baran, P.S. New Synthetic Technology for the Rapid Construction of Novel Heterocycles - Part 1: The Reaction of Dess-Martin Periodinane with Anilides and Related Compounds. Angew. Chem. Int. Ed. Engl. 2000, 39, 622-625.

131. Nicolaou, K.C.; Baran, P.S.; Zhong, Y.L.; Barluenga, S.; Hunt, K.W.; Kranich, R.; Vega, J.A. Iodine(V) Reagents in Organic Synthesis. Part 3: New Routes to Heterocyclic Compounds via o-Iodoxybenzoic Acid-Mediated Cyclizations: Generality, Scope, and Mechanism. J. Am. Chem. Soc. 2002, 124, 2233-2244.

132. Caddick, S.; Hamza, D.; Wadman, S.N. Solid-phase intermolecular radical reactions 1. Sulfonyl radical addition to isolated alkenes and alkynes. Tetrahedron Lett. 1999, 40, 7285-7288.

133. Plourde Jr, R.; Johnson Jr, L.L.; Longo, R.K. Solid-Phase Free Radical Addition of Thiols to Allyl Ethers. Synlett 2001, 2001, 439-441.

134. Timár, Z.; Gallagher, T. Constructing the 2-(thiobenzyl)ethyl carbamate linker via thiyl radical addition. Tetrahedron Lett. 2000, 41, 3173-3176.

135. Dublanchet, A.C.; Lusinchi, M.; Zard, S.Z. Xanthates and solid-phase chemistry. A new soluble polymer analogue of Wang resin. Tetrahedron 2002, 58, 5715-5721.

136. Back, T.G.; Zhai, H. Cyclizations and cycloadditions of acetylenic sulfones on solid supports. Chem. Commun. (Camb.) 2006, 21, 326-328.

137. Attardi, M.E.; Taddei, M. The Barton radical decarboxylation on solid phase. A versatile synthesis of peptides containing modified amino acids. Tetrahedron Lett. 2001, 42, 3519-3522.

138. Koley, D.; Colon, O.C.; Savinov, S.N. Chemoselective Nitration of Phenols with tert-Butyl Nitrite in Solution and on Solid Support. Org. Lett. 2009, 11, 4172-4175.

Sample Availability: not available.

(C) 2011 by the authors; licensee MDPI, Basel, Switzerland. This article is an open access article distributed under the terms and conditions of the Creative Commons Attribution license (http://creativecommons.org/licenses/by/3.0/). 